\title{
CATALOGUE OF SPIDERS (ARANEAE) DEPOSITED IN THE CROATIAN NATURAL HISTORY MUSEUM
}

\author{
Irena Grbac ${ }^{1, *}$, Luka Katušić ${ }^{2}$ \& Marko Lukić ${ }^{3}$ \\ ${ }^{1}$ Department of Zoology, Croatian Natural History Museum, Demetrova 1, \\ HR-10000 Zagreb, Croatia (e-mail: Irena.Grbac@hpm.hr) \\ ${ }^{2}$ Croatian Arachnological Society “Narcis Damin” (e-mail: luka.katusic1@gmail.com) \\ ${ }^{3}$ Croatian Biospeleological Society, Demetrova 1, HR-10000 Zagreb, Croatia \\ (e-mail: marko.lukic@hbsd.hr)
}

Grbac, I., Katušić, L. \& Lukić, M.: Catalogue of spiders (Araneae) deposited in the Croatian Natural History Museum. Nat. Croat. Vol. 28, No. 1., 185-269, Zagreb, 2019.

The paper presents information on the spider collection (Araneae) housed in the Natural History Museum in Zagreb, Croatia. The collection was founded in 1931 and now includes the material assembled in 1884-1964; it consists of 2,461 lots and numbers 8,478 specimens belonging to 464 species, mainly collected by the Croatian arachnologist Narcis Damin. Most specimens are well-preserved in ethanol. This spider collection does not cover all Croatian regions with the majority of samples $(67 \%)$ originating from coastal regions of east Adriatic. A taxonomic re-examination of the collection has revealed 31 species that are new to the Croatian spider fauna. Spider collecting has been resumed in Croatia recently.

Key words: Museum Collection, Narcis Damin, Rikardo Gasperini

Grbac, I., Katušić, L. \& Lukić, M.: Katalog Zbirke pauka (Araneae) u Hrvatskom prirodoslovnom muzeju. Nat. Croat. Vol. 28, No. 1., 185-269, Zagreb, 2019.

Rad donosi podatke o Zbirci pauka (Araneae) pohranjenoj u Hrvatskom prirodoslovnom muzeju u Zagrebu. Zbirka je osnovana 1931. godine i danas obuhvaća materijal prikupljan u razdoblju 1884.-1964.; sastoji se od 2.461 inventarne jedinice te broji 8.478 primjeraka 464 vrste, koje je uglavnom prikupio hrvatski arahnolog Narcis Damin. Većina primjeraka je dobro očuvana u etanolu. Zbirka pauka ne pokriva sve hrvatske regije; većina uzoraka (67\%) potječe s istočne obale Jadrana. Taksonomska revizija zbirke utvrdila je 31 vrstu novu za hrvatsku faunu pauka. Odnedavno je prikupljanje pauka u Hrvatskoj ponovno započelo.

Ključne riječi: muzejska zbirka, Narcis Damin, Rikardo Gasperini

\section{INTRODUCTION}

In the last two hundred years scientific collections form the core of biological research, particularly in taxonomy and systematics. Natural history collections had a crucial role in scientific thoughts of Charles Darwin and Alfred Russell Wallace. Without natural history collections, knowledge on the Earth's biodiversity and benefits that could derive from it would have been lost (Suarez \& TsutsuI, 2004). The Croatian Natural History Museum (CNHM) in Zagreb holds the only museum spider collection in Croatia. 
The requirement of the sustainability of collection can be satisfied if the collection has a legal status and consequently an institution that has been formally tasked with its preservation and meets the technical requirements of storage, regular maintenance and a developed system of information on the deposited materials. The collection value is increased, if a scientific analysis based on collection material and a systematic and planned gathering of specimens are undertaken (Kovačić, 2006; WINKER, 2004). Housed and documented specimens create a scientific potential by ensuring their availability to other researchers (McLean et al., 2016), but the deposition of studied specimens in natural history museums is not a consistent legal requirement yet. Storing collections in museum ensures long-term professional care and continuous public funding financial support for collection maintenance. Preserved specimens and samples of tissues retain a scientific potential for future research applying new technologies such as molecular analysis (McLean et al., 2016). Specimens housed in natural history museums would provide many answers to future questions, but assembling collections require the joint endeavours of curators, scientific community and funding institutions (McLean et al., 2016).

The principal goals of this paper are (1) to present information on and evaluation of the spider collection deposited in the CNHM, and (2) to encourage scientific debates and discussion of future directions in the development of this collection.

\section{MATERIAL AND METHODS}

The nomenclature used in this catalogue follows World Spider Catalog (WSC, 2018). Within each family, the genera and species are arranged alphabetically.

Each entry presents an inventory number (lot) and includes:

- the taxonomic status according to WSC (2018);

- the original taxon name, as given in the ID label;

- specimen numbers (Spec. No.) with reference to sexual maturity: F - adult female, M - adult male, SUBF - subadult female, SUBM - subadult male, JUV juvenile;

- information transcribed from the original data label: leg. = legit (collected by), loc. $=$ locality, date $=$ collection date. If the original locality is poorly known, additional information is given. Abbreviations of overseas localities are indicated in concordance with ISO 3166-1 South America (SA*), AT (Austria), BA (Bosnia and Herzegovina), ME (Monte Negro), IN (India), HU (Hungary), PL (Poland), RO (Romania), SK (Slovakia), SI (Slovenia), RS (Serbia), UA (Ukraine);

- * continental code.

Abbreviations:

* - new species to the Croatian fauna.

*? - dubious records.

** - species represented by damaged specimen, thus their re-identification was not possible. Such species names are listed in their current taxonomic interpretation based on the original identification; if a re-examination did not confirm the species name given originally, such samples are listed under generic names only. 
*** - species represented only with juvenile specimens, thus redetermination was possible only to the genus level. The stated species name is current taxonomic interpretation of the original species name.

\# - quotations the Museum's Spider Catalogue without specimen.

When more than one species was found in a sample, a new inventory number was assigned and specimen(s) were stored in a separate tube. We have added 25 obviously historical inventory notes which had no voucher specimens, thus without assigning a new inventory number to them (\#) .

Dried specimens were re-hydrated. The conventional method (Joceue, 2008; BACCALONI, 2012) involves treating dried specimen with trisodium phosphate $\left(\mathrm{Na}_{3} \mathrm{PO}_{4}\right)$ and re-conservation then in a sufficient amount of ethanol. Trisodium phosphate softens the inner tissues and restores them to the original form. Dried specimens were dipped into a $0.5 \%$ solution of $\mathrm{Na}_{3} \mathrm{PO}_{4}$ until they sank, which was the sign of successful rehydration. After washing in distilled water, specimens were soaked in gradually increasing concentrations of alcohol $(20 \%$ and $40 \%$ ethanol solution). Finally, spiders were preserved in $70 \%$ ethanol in cleaned original glass jars. The permeability of the original ground glass stoppers has been reduced with a layer of beeswax.

\section{RESULTS \& DISCUSSION}

\section{Historical background}

The collection was founded in 1931 by the former curator Nikola Fink, who processed the housed material and compiled the Spider Catalogue (ČAnAĐIJA, 1974) containing 2,242 samples The first 130 specimens came from the collection by Rikardo Gasperini (1853-1939) (ČanaĐija, 1974; ČanaĐija \& Pavletić, 1976; CNHM archive). The Spider Collection of Narcis Damin (1845-1905) arrived to CHNM in 1906 (CNHM archive, annual report 1907). The Damin collection

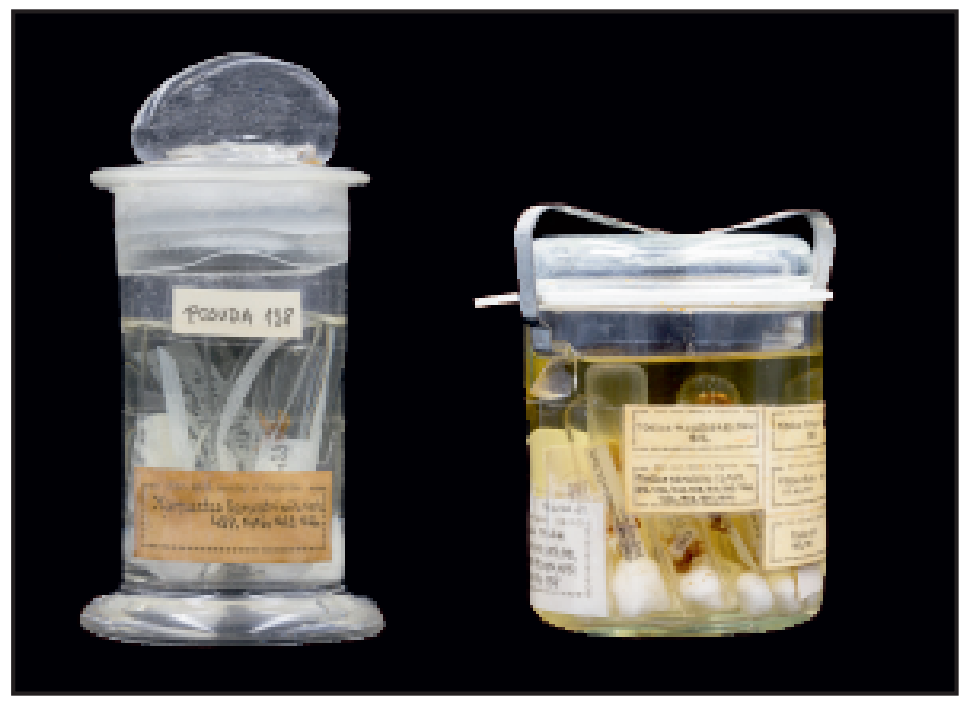

Fig. 1. Specimens in the Spider Collection preserved in ethanol (Photo N. Borčić). 


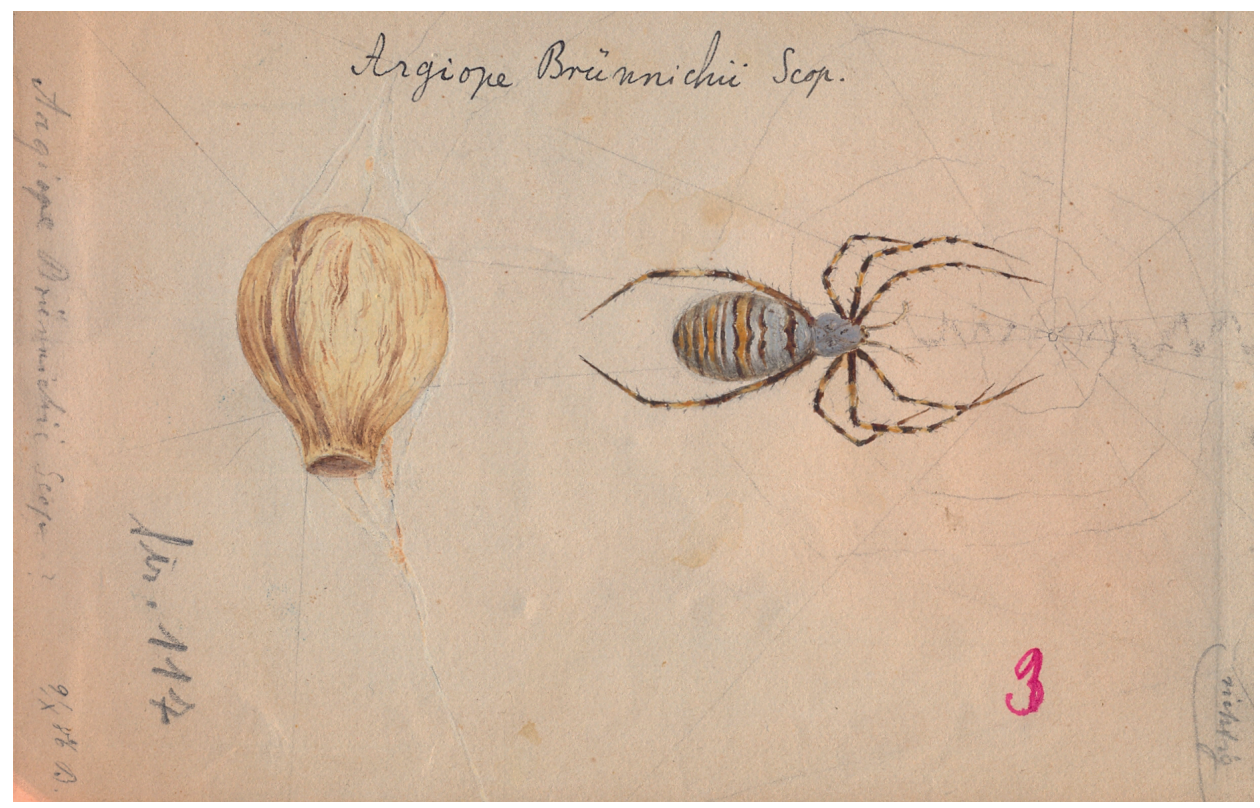

Fig. 2. A page from drawing collection by N. Damin.

contained specimens from two suborders, 21 families, 120 genera and 545 (sub) species and varieties housed in glass jars (Fig. 1), but the original specimen number remains unknown. Damin's accompanying drawing collection (Fig. 2) was donated to the museum in 1916 (CNHM archives, annual report 1917). Although the first written data on spiders in Croatia date back to 1774 (ForTIs 1774), first Croatian arachnologists appeared in the late 19th century when the first papers on Croatian spiders were published by Adolf JurinaC $(1886,1887)$ and Rikardo GASPERINI (1891, 1892). The first Croatian arachnologist was Narcis Damin, who focused mostly on spiders of the northern part of East Adriatic. However, many other Croatian entomologists donated him samples from all over Croatia, and soon Damin gathered an extensive collection of spiders (Katušrć \& DRAKŠIĆ, 2011). In 1900, based on his own collection and literature data, Damin published the first comprehensive inventory of Croatian spiders which listed 556 species (DAMIN, 1900).

\section{Reconservation and revision}

The Museum's Catalogue of 1931 contains 2,242 inventory numbers. A revision and re-identification of the material have revealed 8,478 specimens in 2,461 lots, each with an individual inventory number. The Museum's spider collection holds 464 species, 200 genera and 38 families (Tab. 1). Of them, 349 species were collected from 203 different localities in Croatia (Fig. 3, Appendix 1). The remaining 74 species were sampled from 36 overseas localities (Fig. 4). The names of 35 localities were impossible to confirm.

Specimens in the spider collection are preserved in ethanol and housed in glass jars. Most of them are well-preserved, but in a few cases alcohol evaporated and 
Tab. 1. Number of spider species in the CHNM Collection.

\begin{tabular}{|c|c|c|}
\hline Family & Genus & Species \\
\hline Agelenidae & 8 & 20 \\
\hline Amaurobiidae & 2 & 7 \\
\hline Anyphaenidae & 1 & 2 \\
\hline Araneidae & 19 & 44 \\
\hline Clubionidae & 1 & 17 \\
\hline Corinnidae & 1 & 1 \\
\hline Cybaeidae & 1 & 1 \\
\hline Dictynidae & 4 & 7 \\
\hline Dysderidae & 3 & 9 \\
\hline Eresidae & 1 & 1 \\
\hline Eutichuridae & 1 & 6 \\
\hline Filistatidae & 2 & 2 \\
\hline Gnaphosidae & 17 & 36 \\
\hline Hahniidae & 2 & 2 \\
\hline Linyphiidae & 23 & 32 \\
\hline Liocranidae & 2 & 3 \\
\hline Lycosidae & 12 & 54 \\
\hline Mimetidae & 2 & 3 \\
\hline Nemesiidae & 2 & 3 \\
\hline Oecobiidae & 2 & 2 \\
\hline Oxyopidae & 1 & 4 \\
\hline Philodromidae & 3 & 20 \\
\hline Pholcidae & 5 & 6 \\
\hline Phrurolithidae & 1 & 1 \\
\hline Pisauridae & 2 & 3 \\
\hline Salticidae & 32 & 64 \\
\hline Scytodidae & 1 & 1 \\
\hline Segestriidae & 1 & 4 \\
\hline Sicariidae & 1 & 1 \\
\hline Sparassidae & 2 & 3 \\
\hline Tetragnathidae & 4 & 11 \\
\hline Theridiidae & 23 & 44 \\
\hline Thomisidae & 12 & 37 \\
\hline Titanoecidae & 1 & 3 \\
\hline Trachelidae & 1 & 1 \\
\hline Uloboridae & 2 & 3 \\
\hline Zodariidae & 1 & 4 \\
\hline Zoropsidae & 1 & 2 \\
\hline 38 & 200 & 464 \\
\hline
\end{tabular}




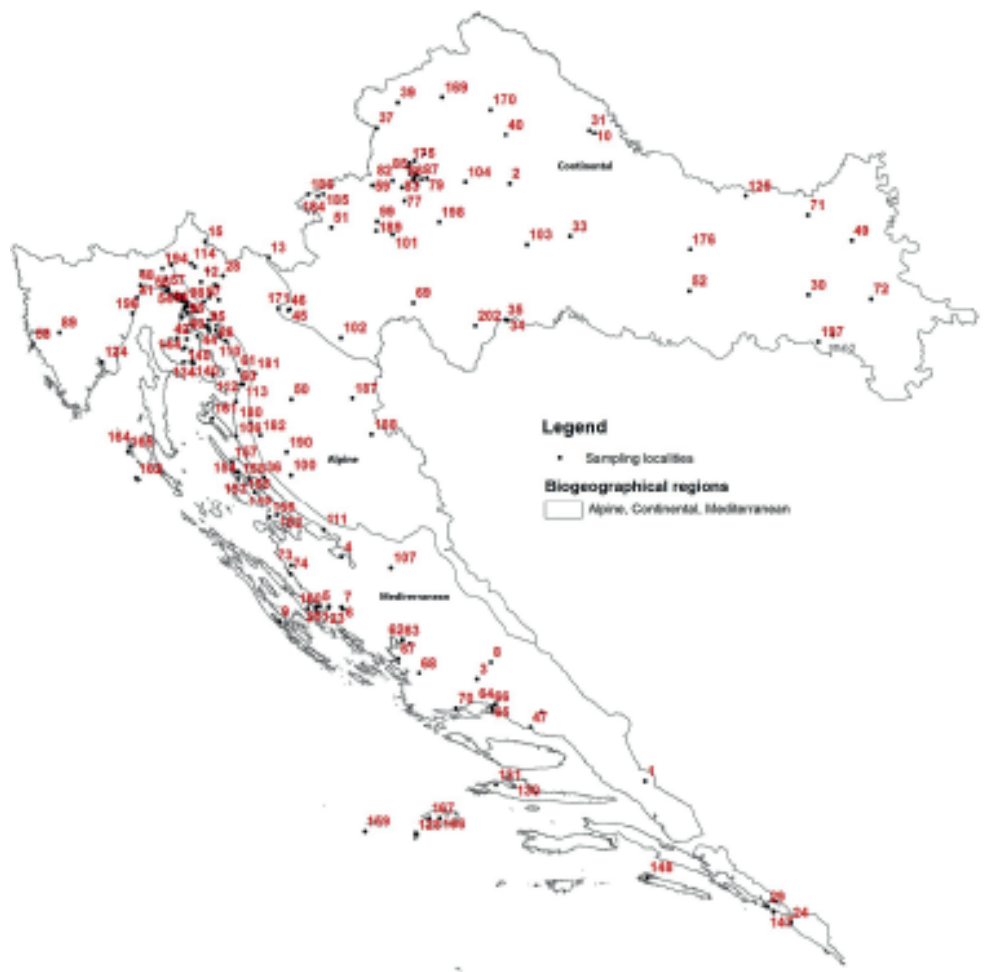

Fig. 3. Map of Croatia with sampling localities.

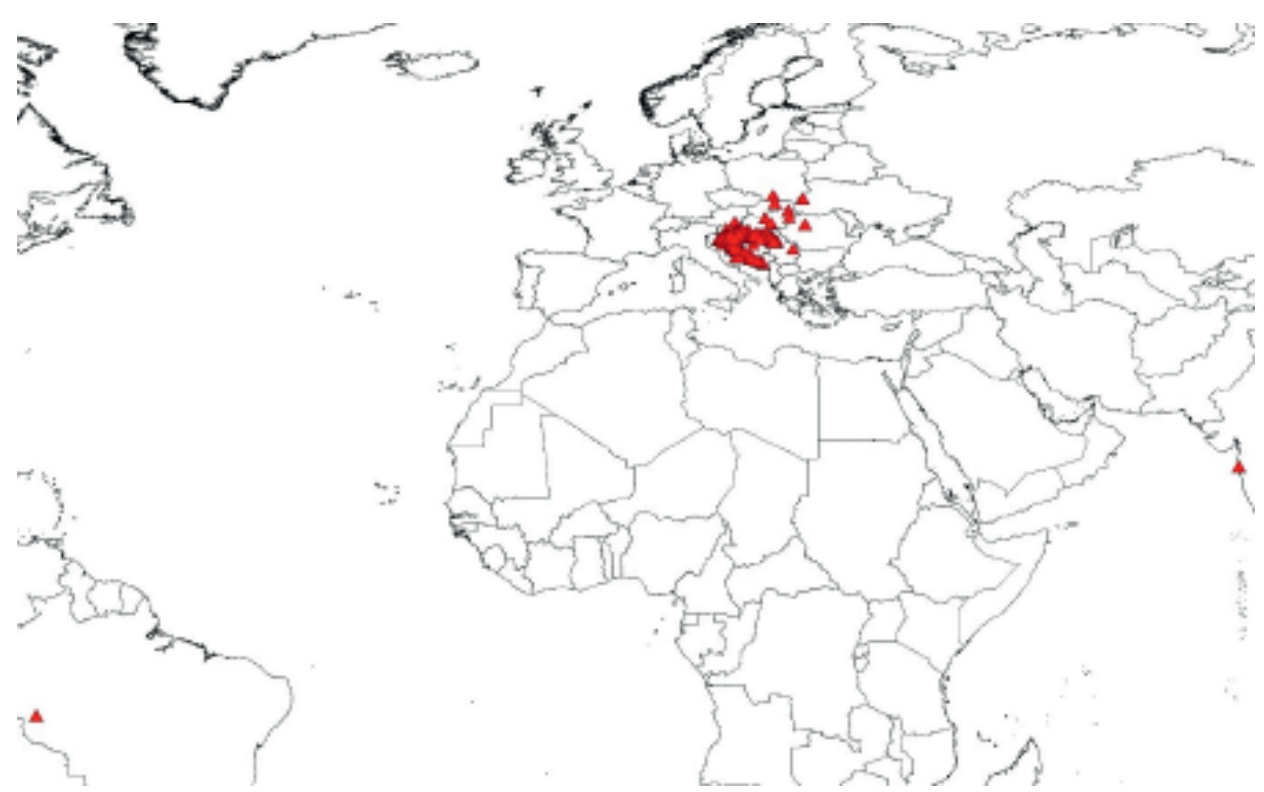

Fig. 4. World map with sampling localities. 
specimens dried out. Such specimens have been re-hydrated. The entire spider collection has been digitized, re-conserved and restored (if needed), and all the specimens have been re-examined and re-identified by L. Katušić.

\section{Collectors and date range}

The oldest sample in the spider collection is Simitiodion simile collected by Damin in 1884. The latest sample was collected in 1964, since then spiders had not been collected and/or deposited in the museum until the collecting activity was resumed in 2017. The newly collected materials are not included in the present work.

Analysis of data from labels and catalogue revealed that among 2,461 lots (=inventory numbers), 43\% ( $\mathrm{N}=1,069)$ have a valid collecting year and other $57 \%(\mathrm{~N}=1,392)$ contain incomplete data sets, with an unknown year. The most productive seasons were 1890/91, due to activity of N. Damin, followed by the period 1897-99 when he and A. Langhoffer were actively collecting, and by 1902

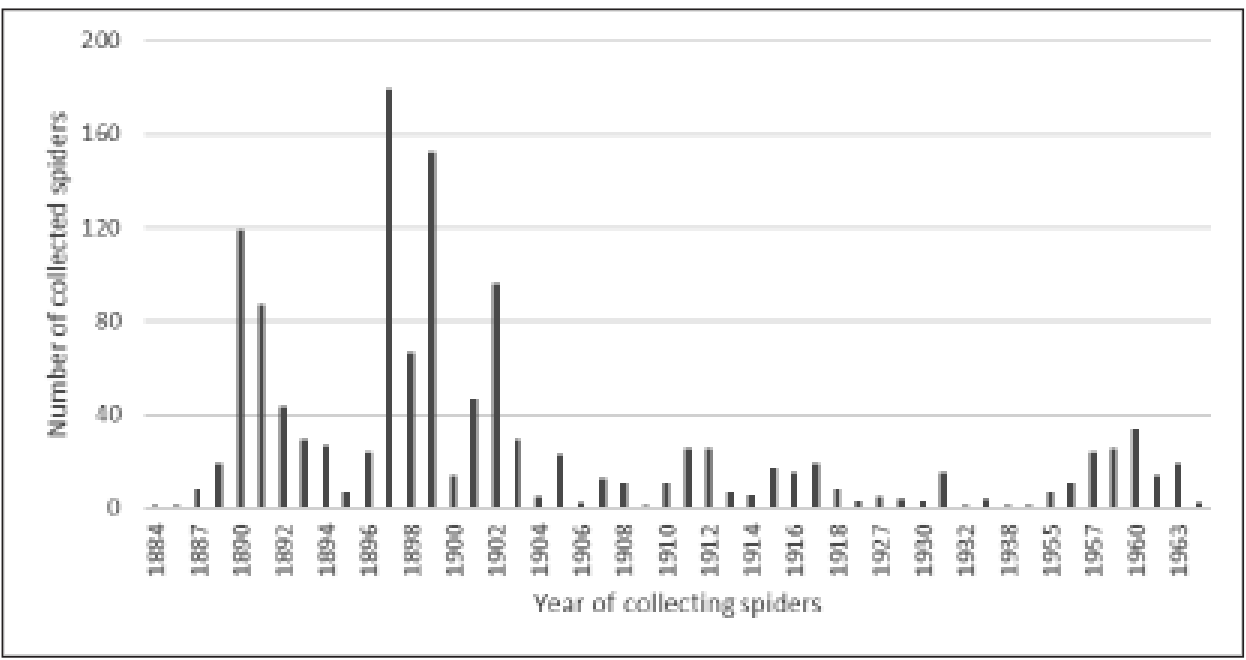

Fig. 5. Number of collected spider specimens at different years.

when various collectors contributed (Fig. 5). Katušić and Drakšić in their historic review of arachnology in Croatia (KATUŠIć \& DRAKšIć, 2011) marked the end of 19th century as the most productive period, corresponding with the highest number of samples accumulated in the collection.

With regards to contribution of various collectors, only 39\% (950) of data contain a collector name, while collectors of 59 samples $(\mathrm{N}=1,511)$ are unknown. Among 35 collectors, N. Damin expectedly contributed the largest sample number - 664. R. Gasperini collected 92 and A. Langhoffer 71 samples, respectively. A. Langhoffer was the second director of the former Zoological Museum, but the majority of his samples were collected before that period. R. Gasperini was the first author who published a list of 195 spider species occurring in Dalmatia (coastal part of Croatia) (GAsperini, 1891, 1892). N. Damin was considered the 
most notable arachnologist in Croatia (Katušić \& DraKšIć 2011). He published an extensive list of 556 spider species occurring in Croatia (DAMIN, 1900), collected and housed vouchers in his spider collection which then formed a significant part of the spider collection at CNHM.

\section{Geographical distribution}

With regards to geographic information, 1,803 samples originated from Croatia, 223 from abroad and 436 samples are without any locality data (Appendix 1, Figs 3 and 4). It is worth noticing that the spider collection was not sampled in order to cover all Croatian regions, but was rather assembled as a result of random sampling and occasional donations. Analysis of the geographic distribution of spiders collected from Croatia shows that majority of them originated from the Mediterranean $(67 \%, \mathrm{~N}=1,201), 21 \%(\mathrm{~N}=383)$ from the mainland and only $12 \%(\mathrm{~N}=219)$ from the Alpine biogeographical region. More than a half of the Mediterranean samples (64\%) were collected from the northern Croatian coast (including Krk island) with the town of Bakar as a single locality with the largest number of samples $(\mathrm{N}=322)$. It is hardly surprising, as N. Damin worked at the Naval school in Bakar. On the other hand, Istra (the northern Croatian coast) is presented with fewer samples, of which 249 originated from the southern Croatian coast (Dalmatia) and islands. Samples from the continental region were mostly taken from two big cities, Osijek ( $N=120)$ and Zagreb (N=112). The CNHM is situated in Zagreb, and the material from Osijek was presumably collected by A. Langhoffer who worked there. The Alpine region is represented with the least sample number. The greatest part was originated from Gorski kotar county, the highland region in the north-western part of Alpine Croatia, whereas the smallest numer originated from Plitvice (Lika region).

Based on the present findings, the spider collection at hand can be considered as historical (the last spider was collected in 1964), with a scattered geographical distribution of samples. Regions that are well-represented are Sjeverno Hrvatsko primorje (North Croatian littoral), Gorski kotar (Alpine region) and the cities of Zagreb and Osijek. However, large parts of Croatia, such as Istra, Adriatic islands, central and southern Dalmatia in the Mediterranean, and the extended region of Dinaric carst, remain almost completely unexplored. The continental region of Croatia was also poorly sampled, except for Zagreb and its vicinity, and Osijek.

\section{Rare and interesting species}

Besides numerous taxonomically interesting records, the spider collection housed in CNHM contains 31 spider species that are new to the Croatian fauna (Appendix 2). The majority of them are widespread in Europe and their presence in Croatia was expected. In some cases, distribution ranges of some species are broadened. For instance, Micaria pallipes, Thanatus coloradensis and Xysticus gallicus are first recorded from Croatia, with published records for the Western Balkans only. The record of M. pallipes from the northern Adriatic shows the gap in the species' Mediterrannean distribution. The range of Palaeartic T. coloradensis is broadened toward the eastern Adriatic. X. tenebrosus is a thomisid species distributed in the Eastern Medditeranean (WSC, 2018), and Croatia can be 
considered as an expected territory of its occurring. Textrix chyzeri from the northern Adriatic extends its somehow scattered distribution range toward the seaside and further to the west. The troglophile Dysderocrates storkani (MAmmola et al., 2017) also recorded from the northern Adriatic extended its range, which includes the SE Balkans, towards the west and possibly to lower altitudes towards the sea. Its data label says above Senj (seaside town), without an altitude specification. Two juvenile specimens identified as Clubiona decora could not be confirmed. This species is limited to Madeira, Porto Santo and Azores (Bosmans et al. 2017b), and those records are probably misidentification. An article describing in detail the importance of spider collection with thorough comments on all taxonomically and faunistically interesting records, including remarks on a possibly new Berinda species, is in preparation by L. Katušić with co-authors.

In the last decade new interest in the arachnology has evolved in the Croatian scientific community. The present catalogue of the spider collection at CNHM represents the first step and baseline for future research. We encourage future researchers whose projects include collecting wild organisms to consider depositing their voucher specimens in the Museum of Natural History Collections in Zagreb. Spider collecting at CNHM was resumed in 2017, and hopefully will result in a better collection access.

\section{SPECIES LIST}

\section{Agelenidae C. L. Koch, 1837}

\section{Agelena labyrinthica (Clerck, 1757)}

INV.NO.417 (2 F): Agelena labyrinthica CI., Povile, 09.08.1899., Damin. INV.NO.418 (1 $\mathrm{JUV}^{*}, 1$ SUBF$*, 2$ M, 3 F): Agelena labyrinthica CI., Gasperini. INV.NO.420 (1 F): Agelena labyrinthica CI., Draga, 09.08., Damin. INV.NO.1343 (1 F): Agelena labyrinthica CI., Kraljevica, 31.07.1899. INV.NO.1348 (1 JUV*): Agelena labyrinthica CI. Dol., Grižane, 14.04. INV.NO.1865 (1 F): Micaria Rogenhoferi O. Herm., Novi Vinodol, 1896., J. Muzler. INV. NO.2122 (1 F): Agelena labyrinthica CI., Kolansko blato, 04.10.1957. INV.NO.2211 (3 F, 1SSP*): Agelena labyrinthica CI., Kotor (Vermač), 19.07.1931. INV.NO.2235 (2 F): Agelena labyrinthica CI., Babac (otok kod Filipjakova), 04.08.1927., Tonko Šoljan.

\section{Agelena sp.}

INV.NO.1545 (1 JUV, 1 SUBM): Epeira sp., Delnice, 5.7.1891. INV.NO.1877 (1 SUBM): Chiracanthium sp., Brezovica, 18.06.1903. INV.NO.1883 (1 JUV): Micaria pulicaria Sund., Ušće Raše, 31.05.1896.

\section{Allagelena gracilens (C. L. Koch, 1841)}

INV.NO.419 (3 M, 6 F): Agelena similis Keys. Dol, 16.08., Damin. INV.NO.1333 (3 JUV*): Agelena similis Keys., Zagreb, 09.07.1902. INV.NO.1338 (3 F): Agelena similis Keys., Topusko, 08.1897. INV.NO.2111 (10 SUBF*): Agelena sp., Caska, 09.06.1958. INV.NO.2114 (4 SUBM ${ }^{*}$ ): Agelena sp., Poljani, 01.07.1960. INV.NO.2193 (4 SUBF*): Agelena sp., Velo blato, o. Pag, 04.07.1956.

\section{Coelotes sp.}

INV.NO.1356 (1 JUV): Coelotes sp., Bukovnik, 10.8.1902. INV.NO.1362 (4 JUV): Coelotes sp., Bitoraj-podnožje, 26.6.1892. 
Histopona luxurians (Kulczyński, 1897)

INV.NO.314 (1 JUV*, 3 F): Tegenaria luxurians Kulcz.Bakar, Damin. INV.NO.1360 (1 F, $\left.7 \mathrm{JUV}^{*}\right)$ : Tegenaria luxurians Kulcz., Vrata.

Histopona torpida (C. L. Koch, 1837)

INV.NO.311 (3 F): Tegenaria torpida C.L. Koch, Bakar, Damin. INV.NO.1329 (1 F): Tegenaria torpida C.L. Koch, Ogulin.

Inermocoelotes anoplus (Kulczyński, 1897)

INV.NO.374 (1 F): Coelotes anoplus Kulcz. INV.NO.375 (1 M): Coelotes inermis L. Koch, Bakar (Kalvar.), 21.10.1890., Damin. INV.NO.1336 (1 M): Coelotes anoplus Kulcz., Bakar, 27.01.1891. INV.NO.1341 (1 M): Coelotes anoplus Kulcz., Žakalj, 17.09. INV.NO.1869 (1 F): Clubiona terrestris Westr., Zagreb, 08.12.

Inermocoelotes gasperinii (Simon, 1891)

INV.NO.217 (1 JUV*, 1 M, 3 F ): Coelotes Gasperinii Sim., Split, Damin. INV.NO.218 (7 M, 14 F): Coelotes Gasperinii Sim., INV.NO.2019 (2 M): Coelotes Gasperinii Sim., Split.

Inermocoelotes inermis (L. Koch, 1855)

INV.NO.375 (2 JUV*): Coelotes inermis L. Koch, Bakar (Kalvar.), 21.10.1890., Damin. INV.NO.1351 (1F): Coelotes sp., Bitoraj-podnožje, 26.6.1892. INV.NO.1866 (1 F): Zilla Keyserlingii Auss., Lokve, 26.06.1910. INV.NO.2016 (2 M): Coelotes inermis L. Koch, Mrkvišće, 02-08.07.1892. INV.NO.2237 (2 F): Coelotes inermis L. Koch, Lokve (Bukovac), 07.1929.

Maimuna vestita (C. L. Koch, 1841)

INV.NO.1028 (1 JUV*, 2 M, 4 F): Textrix vestita C. Koch., Stari grad, Damin. INV.NO.1029 (3 M, 3 F): Textrix vestita C. Koch, Split, Damin. INV.NO.1030 (3 F): Textrix vestita C. Koch. Vis, Damin. INV.NO.1031 (55 F, 3 SUBM*, 5 JUV*, 33 M): Textrix vestita C. Koch, Gasperini. INV.NO.1374 (1 JUV*): Textrix vestita C. Koch, Stari grad, otok Hvar, 29.7. INV.NO.2002 (1 M, 1 F): Textrix vestita C. Koch, Split.

Malthonica sp.

INV.NO.317 (1 JUV): Tegenaria domestica CL. Koch, Fužine, Damin.

Tegenaria bosnica Kratochvíl \& Miller, 1940

INV.NO.313 (1 M): Tegenaria tridentina C., Koch.

Tegenaria campestris (C. L. Koch, 1834)

INV.NO.384 (3 JUV*): Tegenaria campestris C.L. Koch, Sv. Juraj, Damin. INV.NO.2054 $\left(1 \mathrm{JUV}^{*}, 1 \mathrm{M}, 1 \mathrm{~F}\right)$ : Tegenaria campestris C.L. Koch.

Tegenaria cf. decolorata Kratochvil \& Miller, 1940

INV.NO.2006 (2 F): Tegenaria sp., Slivanj. šp.

Tegenaria domestica (Clerck, 1757)

INV.NO.169 (3 F, 1 M, 1SSP*): Tegenaria Derhamii Scop., Zemun. INV.NO.315 (2 F): Tegenaria atrica, Pećine (Vrana), 23.7., Damin. INV.NO.316 (1 SUBM*, 1 F): Tegenaria domestica CL. Koch, Zagreb, Damin. INV.NO.390 (1 M, 3 JUV*): Tegenaria Derhamii Scop., Osijek, St. Pazova, Damin. INV.NO.391 (2 JUV*, 2 F): Tegenaria Derhamii Scop., 
Senj, Damin. INV.NO.392 (4M): Tegenaria Derhamii Scop., Križišće, Damin. INV.NO.394 (7 F): Tegenaria Derhamii Scop., Zemun, Damin. INV.NO.1331 (1SSP*): Tegenaria domestica Cl., Zagreb, 8.12.1902., Polić. INV.NO.1335 (1 JUV*): Tegenaria domestica Cl., Križevci. INV. NO.1345 (1 M): Tegenaria Derhamii Scop., Delnice, Kernic. INV.NO.1350 (1 M, 4 F): Tegenaria Derhamii Scop., Zemun. INV.NO.1355 (5 JUV*): Tegenaria Derhamii Scop., Topusko, 8.1891. INV.NO.2009 (2 F): Tegenaria Derhamii Scop., Sv. Juraj. INV.NO.2015 (1 JUV*): Tegenaria domestica Cl., Fužine. INV.NO.2056 (2 F, 1 M, 1SSP*): Tegenaria Derhamii Scop.

Tegenaria ferruginea (Panzer, 1804)*

INV.NO.1335 (1 M, 2 F): Tegenaria domestica Cl., Križevci. INV.NO.1340 (1 F): Tegenaria domestica Cl., Delnice, 5.7.1891. INV.NO.2015 (1 F): Tegenaria domestica Cl., Fužine. INV. NO.2037 (1 M, 2 F): Tegenaria domestica Cl.

Tegenaria hasperi Chyzer, 1897

INV.NO.312 (1 F): Tegenaria Hasperi Chyzer.Bakar, Damin. INV.NO.384 (6 F): Tegenaria campestris C.L. Koch, Sv. Juraj, Damin. INV.NO.385 (1 F): Tegenaria campestris C.L. Koch, Bakar, Damin. INV.NO.2289 (2 F): Tegenaria atrica, Pećine (Vrana), 23.7., Damin.

Tegenaria pagana (C. L. Koch, 1840)

INV.NO.387 (1 M, 1 F): Tegenaria pagana C.L. Koch, Bakar, Damin. INV.NO.388 (2 M): Tegenaria pagana C.L. Koch, Dubrovnik, Damin. INV.NO.389 (1 M, 6F): Tegenaria pagana C.L. Koch, Gasperini. INV.NO.393 (1 M): Tegenaria Derhamii Scop., Trsat, 17.9., Damin. INV.NO.1692 (1 JUV*, 1 M): Tegenaria pagana C.L. Koch, Senj. INV.NO.2001 (2 JUV*): Tegenaria pagana C.L. Koch, Pad Krke.

Tegenaria parietina (Fourcroy, 1785)

INV.NO.255 (1 M, $\left.1 \mathrm{SUBM}^{*}, 4 \mathrm{SUBF}^{*}, 6 \mathrm{~F}, 10 \mathrm{JUV}^{*}\right)$ : Tegenaria parietina Fourcr. INV. NO.382 (2 F): Tegenaria parietina Fourcr., Bakar, Damin. INV.NO.383 (1 M, 1 F, 4 JUV*): Tegenaria parietina Fourcr., Lokrum, 19.9., Damin. INV.NO.1330 (1 JUV*): Tegenaria parietina Fourcr. INV.NO.2013 (1 F): Tegenaria parietina Fourcr., Bakar, 08.08.1903., Polić. INV.NO.2174 (1 M, 1 F): Tegenaria parietina Fourcr., o. Susak, 21.06.1962.

Tegenaria silvestris (L. Koch, 1872)

INV.NO.317 (1 F): Tegenaria domestica CL. Koch, Fužine, Damin. INV.NO.386 (1 SUBF*, 1 F): Tegenaria sylvestris L. Koch, Bakar, Damin. INV.NO.1334 (1 F): Tegenaria sylvestris L. Koch, Rječina, 28.5 .

Tegenaria sp.

INV.NO.1337 (2 JUV): Tegenaria sp., Bitoraj-podnožje, 26.6.1892. INV.NO.1339 (1 SUBM): Tegenaria sp., Lokrum. INV.NO.1344 (1 JUV): Tegenaria sp., Kraljevica, 31.7.1899. INV.NO.1349 (1 JUV): Tegenaria sp., Sv. Juraj, 7.7.1899. INV.NO.1354 (1 JUV): Tegenaria sp., Izvor Rječine, 22.5.1890. INV.NO.2084\#: Tegenaria sp. INV.NO.2182 (1 SUBF): Tegenaria sp., Caska, 07.10.1955. INV.NO.2205\#: Tegenaria sp., Pašman, 26.07.1927., T. Šoljan.

Textrix caudata L. Koch, 1872

INV.NO.1027 (1 F): Textrix denticulata Oliv., Dol, Bakarac, Damin. INV.NO.1025 (1 $\mathrm{JUV}^{*}$ ): Textrix albosignata Sim., Lukovo, 9.7.1899., Damin. INV.NO.1026 (2 JUV*): Textrix albosignata Sim., Bakar, Kraljevica, Damin. INV.NO.1359 (2 JUV*): Textrix albosignata Sim., Kraljevica, 31.7.1891. INV.NO.1687 (2 JUV*): Textrix albosignata Sim., Bakar. INV. NO.1744 (1 SUBM*, 3 JUV $^{*}$ ): Textrix albosignata Sim., Bakar. 
Textrix chyzeri de Blauwe, $1980^{*}$

INV.NO.1026 (2 F, 6 M): Textrix albosignata Sim., Bakar, Kraljevica, 13.06. i 07.09., Damin. INV.NO.1359 (1 F): Textrix albosignata Sim., Kraljevica, 31.7.1891. INV.NO.1687 (6 F): Textrix albosignata Sim., Bakar, 2.6.1890.

Textrix denticulata (Olivier, 1789)

INV.NO.1027 (2 JUV*): Textrix denticulata Oliv., Dol, Bakarac, Damin. INV.NO.1375*(1 SUBM$^{*}, 1$ F): Textrix denticulata Oliv., Ogulin, 19.4.1896.

Textrix sp.

INV.NO.1369 (1 JUV): Textrix sp., Sv. Juraj, 7.7.1891.

\section{Amaurobiidae Thorell, 1870}

Amaurobius erberi (Keyserling, 1863)

INV.NO.477 (1 M, 3F): Amaurobius fenestralis Stroem., Bakar, 03.03.1891., Damin N. INV.NO.481 (1 F): Amaurobius Erberi Keys., Lukovo, Damin N. INV.NO.482 (3 F, 5 $\mathrm{JUV}^{*}$ ): Amaurobius Erberi Keys., Damin N. INV.NO.483 (3 F): Amaurobius Erberi Keys., Polača, Damin N. INV.NO.484 (2 JUV*, 10M. 26F): Amaurobius Erberi Keys., Gasperini. INV.NO.485 (5M, 7F): Amaurobius Erberi Keys., Zadar, Damin N. INV.NO.488 (1 M, 1 F): Amaurobius obustus L. Koch, Hundsberg. INV.NO.1172 (1 F, 2 JUV*, 3 M): Amaurobius Erberi Keys., Bakar (pivnica), 21.06. INV.NO.1176 (1 F): Amaurobius Erberi Keys., Kostrena Sv. Barbara, 02.05.1890. INV.NO.1181 (4 F): Amaurobius Erberi Keys., Bakar. INV.NO.1190 (2 F): Amaurobius Erberi Keys. Malinska, 26.03. INV.NO.1195 (3 JUV*): Amaurobius Erberi Keys., INV.NO.1199 (1 JUV*, 1 M, 4 F): Amaurobius Erberi Keys., Bakar (Kanal), 20.03. INV. NO.1226 (4 JUV*): Amaurobius Erberi Keys., Kraljevica, 31.07.1899. INV.NO.1688 (1 JUV*): Amaurobius Erberi Keys., Bag, 14.07. INV.NO.2441 (1 F): Coelotes Gasperinii Sim., Split.

Amaurobius fenestralis (Ström, 1768)

INV.NO.477 (3 JUV*): Amaurobius fenestralis Stroem., Bakar, 03.03.1891., Damin N. INV.NO.478 (1 SUBM*, 2 F): Amaurobius fenestralis Stroem., Ratulje, Damin N.

Amaurobius ferox (Walckenaer, 1830)

INV.NO.479 (1 JUV*, 2 M): Amaurobius ferox Walck., Zemun, 1899. INV.NO.480 (1 F, 2 M): Amaurobius ferox Walck., Krk. INV.NO.1237 (2 F, 1 JUV$\left.^{*}, 1 S S P^{*}\right)$ : Amaurobius ferox Walck., Bakar-kanal, 20.03., Damin N. INV.NO.1864 (2 F): Clubiona sp., Brod, 12.05.1900.

Amaurobius jugorum L. Koch, $1868^{*}$

INV.NO.1863 (1 F): Chiracanthium sp., Šestine, 10.12.1916. INV.NO.1887 (1 M): Chiracanthium sp., Krapina, 01.11.1916.

Amaurobius obustus L. Koch, 1868

INV.NO.487 (2 JUV*, 2SUBM*): Amaurobius obustus L. Koch, Bakar, Damin N. INV. NO.488 (1 JUV*): Amaurobius obustus L. Koch, Hundsberg. INV.NO.1247 (1 JUV*, 1 F): Amaurobius obustus L. Koch, Bitoraj podnožje, 26.06.1892.

Amaurobius pallidus L. Koch, 1868

INV.NO.2040 (1 F, 1 JUV**): Amaurobius pallidus L. Koch. 
Amaurobius sp.

INV.NO.1212 (1SSP*): Amaurobius sp., Grižane, 14.04.1900. INV.NO.1887 (2 JUV): Chiracanthium sp., Krapina, 01.11.1916. INV.NO.2293 (1 SUBM): Lycosa morosa L. Koch, Kostrena, Sv. Barb., 2.5.1890.

Callobius claustrarius (Hahn, 1833)

INV.NO.2041 (1 JUV*, 1 M, 2 F): Amaurobius claustralius Hahn.

\section{Anyphaenidae Bertkau,1878}

Anyphaena accentuata (Walckenaer, 1802)

INV.NO.769 (6 F): Anyphaena accentuata Walck., Bakar, 12.04.1892., Damin. INV. NO.770 (3 JUV*): Anyphaena accentuata Walck., Topusko, 08.1891., Damin. INV.NO.771 (1 F): Anyphaena accentuata Walck., Jelenje, 02.07.1893., Damin. INV.NO.1402 (1 JUV*): Anyphaena accentuata Walck., Mali grad. INV.NO.1403 (1 JUV*): Anyphaena accentuata Walck., Rijeka, 01.10.1901. INV.NO.1413 (1 F): Anyphaena accentuata Walck., Zagreb, 1898. INV.NO.1418 (1 SUBF*): Anyphaena accentuata Walck., Plitvice, 27.08.1902. INV.NO.1424 $\left(1 \mathrm{JUV}^{*}\right)$ : Anyphaena accentuata Walck., Plitvice, 27.08.1902. INV.NO.1428 (1 F, $\left.14 \mathrm{JUV}^{*}\right)$ : Anyphaena accentuata Walck., Topusko, 08.1891. INV.NO.1891 (10JUV*): Anyphaena accentuata Walck., Topusko. INV.NO.2350 (3 JUV*): Anyphaena accentuata Walck., Bakar, 12.04.1892., Damin.

\section{Anyphaena sabina L. Koch, 1866}

INV.NO.772 (1 JUV*): Anyphaena sabina L. Koch, Gasperini. INV.NO.773 (1 F): Anyphaena sabina L. Koch, Bakar, Damin. INV.NO.2349 (5M, 5F): Anyphaena accentuata Walck., Bakar, 12.04.1892., Damin. INV.NO.2354 (1 M, 1 F): Anyphaena accentuata Walck., Jelenje, 02.07.1893., Damin.

Anyphaena sp.

INV.NO.1411 (1 JUV): Anyphaena sp., Bitoraj-podnožje, 26.06.1892. INV.NO.1433 (3 JUV): Anyphaena sp., 1901., Z. Bučan.

\section{Araneidae Clerck, 1757}

Aculepeira armida (Audouin, 1826)

INV.NO.443 (3 F): Epeira ceropegia Walck. INV.NO.1855 (1 F): Epeira adianta Walck., Novalja st. (Pag), 14.07.1908., K. Babić. INV.NO.2089 (1 F): Epeira Victoria Thor., Kolansko blato, o. Pag, 27.06.1960. INV.NO.2166 (1 F): Epeira Victoria Thor., Barbat, 22.06.1960.

Aculepeira ceropegia (Walckenaer, 1802)

INV.NO.230 (2 F): Epeira ceropegia Walck., Starigrad (Dalm.), Damin. INV.NO.1842 (1 F): Epeira ceropegia Walck., Vis, 13.06.1910., Babić.

Agalenatea redii (Scopoli, 1763)

INV.NO.231 (2 F): Epeira Redii Scop., Zemun. INV.NO.233 (3 F): Epeira Redii Scop., Novi, 15.4.1897. INV.NO.322 (2 JUV*): Epeira Redii Scop., Zagreb, 1895. INV.NO.441 (1 JUV*, 1 M, 9F): Epeira Redii Scop., Bakar. INV.NO.442 (1 F): Epeira Redii Scop., Vrbica (Dalm.). INV.NO.454 (7 F): Epeira Redii Scop., INV.NO.1523 (2 JUV*): Epeira Redii Scop., Novi, 08.04.1898. INV.NO.1626 (1 JUV*, 8F): Epeira Redii Scop., Primorje. INV.NO.1635 (1 $\left.J_{U V}^{*}\right)$ : Epeira Redii Scop., Trsat. INV.NO.1637 (1 JUV*): Epeira Redii Scop., Bag. 
Araneidae gen. sp.

INV.NO.2415 (1 JUV*): Pisaura mirabilis CI., Kraljičin zdenac, 17.07.1897., Langhoffer. INV.NO.2440 (1 JUV*): Pholcus opilionides Schr., Buk Krke.

Araneus alsine (Walckenaer, 1802)

INV.NO.238 (3 JUV*): Epeira alsine Walck., Osijek, Damin. INV.NO.1458 (1 M): Epeira alsine Walck. INV.NO.1622 (1 JUV*): Epeira alsine Walck., Zagreb, 10.4.1916. INV.NO.1673 $\left(1 \mathrm{JUV}^{*}\right)$ : Epeira alsine Walck., Jelenje, 2.7.1893.

Araneus angulatus Clerck, 1757

INV.NO.249 (1 F, 1 M): Epeira angulata Clerck, Ogulin, Damin. INV.NO.250 (2 F): Epeira angulata Clerck, Split, Damin. INV.NO.1611 (1 F): Epeira pallida?, Ogulin. INV.NO.2133 (1 SUBM ${ }^{*}$ : Epeira angulata Clerck, Caska, 26.06.1962. INV.NO.2149 (1 SUBF*): Epeira angulata Clerck, Gaska, o. Pag, 24.6.1960. INV.NO.2204 (7 F): Epeira angulata Clerck, Vermač (Kotor), 19.07.1931. INV.NO.2444*(1 F): Epeira angulata Clerck, Kotor, 01.07.1931. INV.NO.2445 (4 SUBF*): Epeira angulata Clerck, Kotor, 01.07.1931. INV.NO.2461 (1 F): Epeira angulata Clerck, Kotor, 01.07.1931., Ž.

\section{Araneus circe (Audouin, 1826)}

INV.NO.425 (1 JUV* 4 F): Aranea Circe Savign. INV.NO.426 (1 F): Aranea Circe Savign., Lukovo. INV.NO.427 (1 F): Aranea Circe Savign., Palagruža. INV.NO.428 (1 JUV*, 1 SUBM $^{*}$ ): Aranea Circe Savign., Zemun. INV.NO.428 (1 SUBM*, 1 F, 2 JUV*): Aranea Circe Savign., Zemun. INV.NO.429 (1 SUBM*, 4 JUV*): Aranea Circe Savign., Muo, Kotor. INV. NO.452 (2 JUV*): Aranea Circe Savign. INV.NO.455 (1 F, 1 M): Aranea Circe Savign., Bakar, Damin. INV.NO.1469 (4 JUV*): Aranea Circe Savign., Starigrad, 11.07.1899. INV. NO.1477 (1 JUV*): Aranea Circe Savign., o. Košljun. INV.NO.1620 (1 F): Epeira dromedaria Walck., Novi (Kalvarija), 4.7.1894. INV.NO.1676 (5 JUV*): Aranea Circe Savign., Bakar nad Kup. INV.NO.1832 (1 F): Aranea Circe Savign., Novi, vrelo Ivanj, 08.07.1912., Zuber. INV.NO.1833 (1 F): Epeira angulata Clerck., Punta od Mandrača, 22.08.1905. INV.NO.1835 (2 F, 1 M): Aranea Circe Savign., Vis, 13.06.1910., K. Babić. INV.NO.2065 (1 F): Argiope sp., Bag, 19.07.1899. INV.NO.2072 (2 F): Aranea Circe Savign., Novalja (Špital), O. Pag, 06.07.1960. INV.NO.2090 (3 F): Aranea Circe Savign., Kolansko blato, o. Pag, 27.06.1960. INV.NO.2120 (1 F): Aranea Circe Savign., Caska, o. Pag, 24.06.1960. INV.NO.2147 (1 F): Aranea Circe Savign., Unije, 10.09.1963. INV.NO.2163 (1 F): Aranea Circe Savign., Časka, o. Pag, 12.06.1958. INV.NO.2175 (4 F): Aranea Circe Savign., Novalja, Kolansko, 01.07.1955. INV.NO.2178 (1 F): Epeira angulata Clerck., Poljane, 01.07.1960. INV.NO.2209 (1 F): Epeira angulata Clerck., Biograd n/m, 06.07.1927., T. Soljan. INV.NO.2212 (1 F): Epeira angulata Clerck., Šibenik, 11.06.1926., K. Babić. INV.NO.2216 (2 F): Epeira angulata Clerck., Jablanac, 07.1931., K. Babić. INV.NO.2443 (4 F): Epeira angulata Clerck, Kotor, 01.07.1931. INV. NO.2453 (1 M): Aranea Circe Savign., Bakar, Damin.

\section{Araneus diadematus Clerck, 1757}

INV.NO.350\#: Aranea diadema Clerck., Kraljičin zdenac. INV.NO.432 (1 M): Aranea diadema Clerck., INV.NO.432 (1 F): Aranea diadema Clerck., INV.NO.435 (1 F): Aranea diadema Clerck., Šibenik. INV.NO.436 (2 F, 2 M, 6JUV*): Aranea diadema Clerck. INV. NO.1451 (1 M): Epeira alpica L. Koch, Plitvice, 27.08.1902., Šnap. INV.NO.1454 (3 JUV*): Aranea diadema Clerck., Osijek, 12.1898. INV.NO.1455 (1 F): Aranea diadema Clerck., Delnice , 14.08.1902., Vorenjak. INV.NO.1459 (1 JUV*): Aranea diadema Clerck., Bakar (kanal), 23.07.1902. INV.NO.1461 (1 M): Aranea diadema Clerck., Rijeka, 23.09.1897. INV. NO.1466 (1 M, 3 JUV*): Aranea diadema Clerck., Jasikovac, 18.08.1902. INV.NO.1467 (2 
JUV*): Aranea diadema Clerck., Kostrena Sv. Barbara, 02.05.1890. INV.NO.1472 (2 JUV*): Aranea diadema Clerck., Zagreb, 04.07.1902., Peičić. INV.NO.1473 (1 JUV*): Aranea diadema Clerck., Osijek, 1898. INV.NO.1474 (1 JUV*): Aranea diadema Clerck., Crikvenica, 08.08. INV.NO.1490 (3 JUV*): Aranea diadema Clerck., Stari Banj. INV.NO.1491 (1 JUV*): Aranea diadema Clerck., Ogulin. INV.NO.1495 (1 JUV*): Aranea diadema Clerck., Vinodol, 14.04.1897. INV.NO.1500 (1 SUBF*): Aranea diadema Clerck., Fužine, 06.1897. INV. NO.1501 (1 SUBF*, 1 SUBM*): Aranea diadema Clerck., Zagreb. INV.NO.1505 (1 F): Aranea diadema Clerck., Sljeme, 15.09.1898. INV.NO.1510 (1 F): Aranea diadema Clerck., Plitvice, 27.08.1902. INV.NO.1514 (1 F): Aranea diadema Clerck., Bakar, 03.10.1903. INV.NO.1517\#: Aranea diadema Clerck., Bregi. INV.NO.1519 (1SSP*): Aranea diadema Clerck., Križevci. INV.NO.1606 (1 F): Aranea diadema Clerck., Senj, 1896., Dr. Babić. INV.NO.1610 (1 F): Epeira pallida?, Klek, 24.8.1902. INV.NO.1621 (1 F): Aranea diadema Clerck., 03.11.1900. INV.NO.1630 (2 F, 1 M): Epeira pallida?, Jadovno, 08.1905., Dr. Babić. INV.NO.1647 (1 F, $\left.1 \mathrm{JUV}^{*}\right)$ : Aranea diadema Clerck., Plitvice, 18.08.1898. INV.NO.1667 (1 M): Aranea diadema Clerck., Zagreb, 19.09.1910. INV.NO.1803 (1 F): Epeira quadrata Clerck., Osijek, 25.09.1917. INV.NO.1831 (1 F): Aranea diadema Clerck., Klek, 24.08.1902. INV.NO.1839 (2 F, 1 M, 2 JUV $^{*}$ ): Aranea diadema Clerck., Topusko, 08.1891. INV.NO.1856 (2 SUBM ${ }^{*}, 1$ $\mathrm{JUV}^{*}$ ): Aranea diadema Clerck., Grabarje, Alan, 31.05.1914., Langhoffer. INV.NO.2070 $\left(1 \mathrm{JUV}^{*}\right)$ : Aranea diadema Clerck., Zagreb, 05.08.1905. INV.NO.2086\#: Aranea diadema Clerck. INV.NO.2113 (2 F): Aranea diadema Clerck., Novalja, 04.10.1951. INV.NO.2132 (1 F): Aranea diadema Clerck., Unije, 10.09.1963. INV.NO.2137 (1 F): Aranea diadema Clerck., Unije, 1.-10.09.1963. INV.NO.2151 (4 F): Aranea diadema Clerck., Caska, o. Pag, 24.06.1960. INV.NO.2157 (3 F): Aranea diadema Clerck., Novalja, o. Pag, 04.10.1957., Magerle. INV. NO.2160 (7 F): Aranea diadema Clerck., Novalja, o. Pag, 04.10.1957. INV.NO.2190 (21 F): Aranea diadema Clerck., Novalja, Kolansko blato, 04.10.1957. INV.NO.2210 (1 F): Aranea diadema Clerck., Zagreb, 06.10.1931. INV.NO.2240 (1 F): Aranea diadema Clerck., Lokve, 07.1930. INV.NO.2449 (1 F): Aranea diadema Clerck., Zagreb, 10.1916., J. Jalić.

Araneus grossus (C. L. Koch, 1844)

INV.NO.243 (2 F): Epeira grossa C.L. Koch, Bakar, 10.07.1889. i 15.06.1893., Damin. INV.NO.244 (1 F): Epeira quadrata Clerck., Pazarište, 20.06.1931., Š. Erbić. INV.NO.1538 (1 JUV*): Epeira grossa C.L. Koch, Rovinj, 23.9.1902. INV.NO.1605 (1 SUBF*): Epeira grossa C.L. Koch, Ušće Raše, 31.5.1896. INV.NO.1612 (1 F): Epeira grossa C.L. Koch, Senj, 1895. INV.NO.1615 (1 F): Epeira grossa C.L. Koch, Novi Vinodol, J. Muzler.

\section{Araneus marmoreus Clerck, 1757}

INV.NO.323 (1 F): Epeira marmorea for. pyramidalis Clerck., Otočac, 19.09.1915., B. Šarinić. INV.NO.349 (3 F): Epeira marmorea for. pyramidalis Clerck., Osijek, 16.10.1893. INV.NO.431 (4 F): Epeira marmorea Clerck., Susak, Damin. INV.NO.1502 (2 F , 1 JUV*): Epeira marmorea for. pyramidalis Clerck., Delnice, 08.08.1902., Stipetić. INV.NO.1507 (1 $\mathrm{JUV}^{*}$ ): Epeira marmorea Clerck., Topusko, 8.1891. INV.NO.1613 (1 F): Epeira patagiata Clerck., Plešce, 08.1902., Dr. Langhoffer. INV.NO.1674 (8JUV*): Epeira marmorea for. pyramidalis Clerck., Senj. INV.NO.2234 (8 F): Aranea raji.

\section{Araneus quadratus Clerck, 1757}

INV.NO.439 (2 F, 1 M): Epeira quadrata Clerck., Zagreb. INV.NO.1598 (1 F): Epeira Victoria Thor., Vinkovci, 15.9.1903. INV.NO.1607 (1 F): Epeira quadrata Clerck., Garešnica, 25.09.1891. INV.NO.1609 (1 F): Epeira quadrata Clerck., Prečko, 07.09.1902., Vj. Slavnik. INV.NO.2066 (1 F): Epeira sp. Strosina, 10.10.1903., Romić. 
Araneus sp.

INV.NO.1158 (1 JUV): Epeira sp., Zagreb, 1896., Dr. Langhoffer. INV.NO.1509 (2 JUV): Epeira sp., Bakarac, 19.8.1897. INV.NO.1541 (5 JUV): Epeira sp., Borova draga, 3.7. INV. NO.1544 (1 JUV): Epeira sp., Sv. Kuzam, 20.8.1902., Polić. INV.NO.1597 (1 JUV): Misumena vatia Clerck., Plitvice, 25.05.1902., Šnap. INV.NO.2269 (1 JUV): Theridium formosum Clerck., Ogulin, Damin N. INV.NO.2338 (2 JUV): Pisaura mirabilis CI., Kostrena Sv. Barb., 2.5.1900.

Araneus sturmi (Hahn, 1831)

INV.NO.232 (1 JUV*): Epeira Sturmii Hahn., Sv. Filip i Jakov (Dalm.), Damin. INV. NO.234 (1 JUV*): Epeira Sturmii Hahn., Crni lug, Damin. INV.NO.1503 (2 F): Epeira Sturmii Hahn., Fužine, 6.1897. INV.NO.1522 (1 JUV*): Epeira Sturmii Hahn., Osijek, 1897., Dr. Langhoffer. INV.NO.1526 (2 JUV*): Epeira triguttata Fabr. INV.NO.1657 (2 F): Meta Menardi Latr., Lokve-Golubnjak, 06.06.1912., Dr. Langhoffer. INV.NO.1675 (1 F): Epeira Sturmii Hahn., Pod Bitorajem-Vrata, 26.06.1892.

Araniella alpica (L. Koch, 1869)

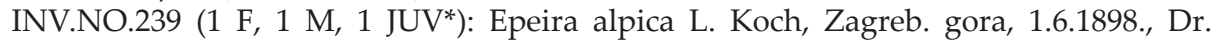
Langhoffer. INV.NO.242 (4 SUBF* $\left.1 \mathrm{SUBM}^{*}, 6 \mathrm{JUV}^{*}\right)$ : Epeira alpica L. Koch, Mrzla vodica, Crni lug. INV.NO.437 (5 F, 1 M, 2 JUV*): Epeira alpica L. Koch, Zagreb. gora., Dr. Langhoffer. INV.NO.438 (1 F): Epeira alpica L. Koch. INV.NO.1482 (1 F, 1 M): Epeira alpica L. Koch, Fužine, 6.6.1897. INV.NO.1682 (1 SUBM* 4 JUV*): Epeira alpica L. Koch, Mrzla vodica.

Araniella cucurbitina (Clerck, 1757)

INV.NO.240 (1 F, 2 M, 4 SUBF*, 3 SUBM*, 3 JUV*): Epeira cucurbitina Clerck., Bakar, Primorje. INV.NO.240 (): Epeira cucurbitina Clerck., Bakar, Primorje. INV.NO.241 (1 JUV*): Epeira cucurbitina Clerck., INV.NO.1438 (2 F, 1 M, 1 SUBF*): Epeira cucurbitina Clerck., Fužine, 11.06.1891., Dr. Langhoffer. INV.NO.1440 (1 F): Epeira cucurbitina Clerck., Kraljičin zdenac, 14.06.1897. INV.NO.1450 (2 JUV*): Epeira cucurbitina Clerck., Novi, 15.04.1899. INV.NO.1457 (1 JUV*): Epeira cucurbitina Clerck., Dol., Grižane, 13.04. INV.NO.1463 (1 F): Epeira cucurbitina Clerck., Plitvice, 17.08.1902. INV.NO.1476 (3 JUV*): Epeira cucurbitina Clerck., Topusko, 08.1891. INV.NO.1481 (1 F): Epeira cucurbitina Clerck., Božjakovina, 28.05.1898. INV.NO.1485 (1 F): Epeira cucurbitina Clerck., Križevci, 04.09.1897. INV. NO.1689 (1 JUV*): Epeira cucurbitina Clerck., Izvor Rječine, 22.05.1890. INV.NO.2027 (1 F): Epeira cucurbitina Clerck. INV.NO.2069 (1 F): Epeira alpica L. Koch, Sv. Gera, 19.06.1917.

Araniella displicata (Hentz, 1847)

INV.NO.1853 (1 F): Epeira alpica L. Koch, Preko Bakar, 28.08.1905.

Araniella inconspicua (Simon, 1874) ****

INV.NO.1735 (1 JUV): Epeira inconspicia E. Sim., Dalmacija.

Araniella opisthographa (Kulczyński, 1905)

INV.NO.1512 (1 M): Singa nitidula C.L. Koch, Ogulin, 4.6.1898. INV.NO.2275 (2 M): Epeira cucurbitina Clerck., Bakar, Primorje.

Araniella sp.

INV.NO.1706 (2 JUV): Epeira sp., Fužine, Damin. 
Argiope bruennichi (Scopoli, 1772)

INV.NO.930 (1 F): Argiope Brünnichii Scop., Zagreb, 1895., Damin. INV.NO.933 (13 M): Argiope Brünnichii Scop., Bakar, Damin. INV.NO.934 (3 F, 1 M, 1 JUV*): Argiope Brünnichii Scop., Bakar, Damin. INV.NO.935 (3 F, 1 JUV*): Argiope Brünnichii Scop. INV. NO.935\#: Argiope Brünnichii Scop., Bakar. INV.NO.937 (3 F): Argiope Brünnichii Scop., Palagruža, 1911. INV.NO.1000 (1 cocoon*): Argiope Brünnichii Scop., Palagruža. INV. NO.1002 (1 cocoon*): Argiope Brünnichii Scop., Vinodol, Damin. INV.NO.1003 (2 F): Argiope sp., Bakar, Damin. INV.NO.1081 (1 SUBM*): Argiope Brünnichii Scop., Zadar, 15.07.1899. INV.NO.1098 (1 JUV*): Argiope Brünnichii Scop., Bakar, 06.08.1902., Polić. INV.NO.1103 (1 SUBF*, 1 SUBM*): Argiope Brünnichii Scop., Bakar (kanal), 23.07.1902. INV.NO.1113 (1 F): Tetragnatha Solandrii Scop., Subotište, 25.7.1902. INV.NO.1614 (1 F): Argiope Brünnichii Scop., Pisarovina, 29.08.1902. INV.NO.1623 (1 F): Argiope Brünnichii Scop., Kostrena Sv. Barb., 28.08.1903., Polić. INV.NO.1627 (1 F): Argiope Brünnichii Scop., Bakar, 03.10.1903. INV.NO.1629 (1 F): Argiope Brünnichii Scop., Bakar, 06.10.1903. INV. NO.1643 (1 F): Argiope Brünnichii Scop., Krasica. INV.NO.1646\#: Argiope Brünnichii Scop., Bakar, 03.10.1903. INV.NO.1829 (2 F): Argiope Brünnichii Scop., Zagreb-Tuškanac, 1887. INV.NO.1834 (2 F): Argiope Brünnichii Scop., Moslavina, 19.08.1915. INV.NO.1837 (3 F): Argiope Brünnichii Scop., Mandrač, 29.08.1905. INV.NO.1847 (1 F): Argiope Brünnichii Scop., ot. Sv. Marko, 16.08.1902. INV.NO.1851 (1 F): Argiope Brünnichii Scop., Pag, 10.1901., Dr. Babić. INV.NO.2073 (5 F): Argiope Brünnichii Scop., o. Susak, 18.06.1962. INV. NO.2074 (6 F): Argiope Brünnichii Scop., Caska, o. Pag, 24.09.1957. INV.NO.2075 (4 F): Argiope Brünnichii Scop., o. Unije, 1-10.09.1963. INV.NO.2139 (1 F): Argiope Brünnichii Scop., Caska, o. Pag, 26.06.1962. INV.NO.2146 (1 M): Argiope Brünnichii Scop., Unije, 1-10.09.1963. INV.NO.2146 (1 F): Argiope Brünnichii Scop., Unije, 1-10.09.1963. INV. NO.2153 (1 F): Argiope Brünnichii Scop., Novalja, o. Pag, 04.10.1957. INV.NO.2156 (1 F): Argiope Brünnichii Scop., Kolansko blato, o. Pag, 04.10.1957. INV.NO.2159 (1 F): Argiope Brünnichii Scop., Novalja, o. Pag, 04.10.1957. INV.NO.2170 (7 F): Argiope Brünnichii Scop., Susak, 16.06.1962. INV.NO.2171 (1 F): Argiope Brünnichii Scop., Caska, o. Pag, 01.10.1957. INV.NO.2206 (1 F): Argiope Brünnichii Scop., Zadar, 08.1926., T. Šoljan. INV.NO.2217 (2 F): Argiope Brünnichii Scop., Rab, 1926., K. Babić. INV.NO.2230 (2 F): Argiope Brünnichii Scop., Draga, 09.08.1908., K. Babić.

\section{Argiope lobata (Pallas, 1772)}

INV.NO.929 (1 F): Argiope lobata Pall., Senj, Damin. INV.NO.931 (1 F, 1 JUV*): Argiope lobata Pall. INV.NO.932 (1 SUBF*): Argiope lobata Pall., Budva (Dalm.), Damin. INV. NO.1001 (3 F): Argiope lobata Pall., Palagruža, 1911. INV.NO.1604 (2 JUV*): Argiope lobata Pall., Pjeski Füllopizallas. INV.NO.1616 (1 F, 1 JUV*): Argiope lobata Pall., Senj, 1896., Dr. Babić. INV.NO.1616 (): Argiope lobata Pall., Senj, 1896., Dr. Babić. INV.NO.1670 (2 F): Argiope lobata Pall., Palanka, 01.09.1902, M. Šnap. INV.NO.1830 (1 F): Argiope lobata Pall., Pag, 10.1911., K. Babić. INV.NO.2081 (1 F): Argiope lobata Pall., Caska, o. Pag, 27.09.1957. INV.NO.2082 (3 F): Argiope lobata Pall., o. Unije, 1-10.09.1963. INV.NO.2155 (1 F): Argiope lobata Pall., Kolansko blato, 04.10.1957. INV.NO.2167 (1 F): Argiope lobata Pall., Caska, o. Pag, 08.06.1956. INV.NO.2187 (1 F): Argiope lobata Pall., Novalja, o. Pag, 01.10.1957. INV. NO.2207 (2 F): Argiope lobata Pall., Corata, otok pred Biogradom, 02.08.1927., T. Šoljan. INV.NO.2213\#: Argiope lobata Pall. INV.NO.2214 (1 F): Argiope lobata Pall. INV.NO.2452 (2 F): Argiope lobata Pall.

Cercidia prominens (Westring, 1851)

INV.NO.161 (1 JUV*): Cercidia prominens Westr., Bakar, Damin. INV.NO.161 (3 F, 3 M, $2 \mathrm{SUBM}^{*}$ ): Cercidia prominens Westr., Bakar, Damin. 
Cyclosa conica (Pallas, 1772)

INV.NO.150 (2 F , 1 M, 1 SUBM*, 1 JUV*): Cyclosa conica Pall. INV.NO.535 (2SUBM*): Cyclosa conica Pall., Osijek, Damin. INV.NO.535 (5 JUV*): Cyclosa conica Pall., Osijek, Damin. INV.NO.536 (1 JUV*): Cyclosa conica Pall., Zagreb, Damin. INV.NO.612 (1 F): Cyclosa conica Pall. INV.NO.1076 (1 M): Cyclosa conica Pall., Fužine, 6.1897. INV.NO.1082 (1 JUV*): Cyclosa conica (sp.) Pall., Zagreb, 1897., Dr. Langhoffer. INV.NO.1088 (2 F): Cyclosa conica Pall. INV.NO.1093 (18 JUV*): Cyclosa conica Pall. INV.NO.1120 (1 M): Cyclosa conica Pall., Osijek, 1897., Dr. Langhoffer. INV.NO.1653 (1 F): Epeira opuntiae, Zagreb, Tuškanac, 14.5.1907.

Gibbaranea bituberculata (Walckenaer, 1802)

INV.NO.25 (1 F): Salticus sp., Bribir-Novi, 15.06.1897. INV.NO.422 (24 F, 7 M, 2 SUBM*, 3 JUV$^{*}$ ): Epeira dromedaria Walck., Bakar, Damin. INV.NO.423 (2 F): Epeira dromedaria Walck., Rijeka (Fiume), 5.6., Damin. INV.NO.430 (1 F, 2 M): Epeira dromedaria Walck., INV.NO.1436 (1 M): Epeira dromedaria Walck., Bribir-Novi, 15.4.1897. INV.NO.1437 (2 $\mathrm{JUV}^{*}$ ): Epeira dromedaria Walck., Rijeka, 23.9.1897. INV.NO.1470 (1 F): Epeira dromedaria Walck., INV.NO.1475 (1 F): Epeira dromedaria Walck., Osijek, 4.1899. INV.NO.1480 (2 F): Epeira dromedaria Walck., Osijek, 1891. INV.NO.1534 (2 F , 1 M): Epeira dromedaria Walck., Kecskemet. INV.NO.1857 (1 JUV*): Epeira dromedaria Walck., Vrata.

Gibbaranea gibbosa (Walckenaer, 1802)

INV.NO.421 (1 JUV*): Epeira gibbosa Walck., Crni lug, Damin. INV.NO.424 (5 JUV*): Epeira gibbosa Walck., Pad Krke. INV.NO.1499 (1 F): Epeira gibbosa Walck., Kecskemet.

Gibbaranea omoeda (Thorell, 1870)

INV.NO.1497 (1 JUV*): Epeira omoeda Thor., Fužine, 07.06.1899., Dr. Langhoffer. INV. NO.1498 (1 M): Epeira omoeda Thor., Fužine, 6.9.1897.

Gibbaranea sp.

INV.NO.25 (1 JUV*): Salticus sp., Bribir-Novi, 15.06.1897. INV.NO.1464 (1 JUV): Epeira angulata Clerck., 12.3.1899. INV.NO.1659 (1 JUV): Epeira angulata Clerck., Sunto, 27.03. INV.NO.2276 (4 JUV): Epeira grossa C.L. Koch, Bakar, 10.07.1889. i 15.06.1893., Damin. INV.NO.2404 (1 JUV): Attus daminii Chyzer., Žakalj, 17.09.

Glyptogona sextuberculata (Keyserling, 1863)

INV.NO.260 (1 F): Glyptogona 6-tuberculata Keys., Budva, 4.8.1894., Damin. INV. NO.1699 (2 JUV*): Glyptogona 6-tuberculata Keys., Polača, o. Mljet.

Hypsosinga albovittata (Westring, 1851)

INV.NO.2042 (1 JUV*): Singa albovittata Westr., INV.NO.1508 (2 F): Singa Herii Hahn. INV.NO.666 (4 SUBM*, 9JUV*): Singa Herii Hahn., Krk, Damin.

Hypsosinga pygmaea (Sundevall, 1831)

INV.NO.1506 (1 F): Singa pygmaea Sund., Osijek, 7.1901. INV.NO.1542 (1 F): Singa pygmaea Sund., Stara Pazova, 10.8.1898.

Hypsosinga sanguinea (C. L. Koch, 1844)

INV.NO.663 (1 F): Singa sanguinea C.L. Koch, Bakar, Damin. INV.NO.1504 (1 F): Singa sanguinea C.L. Koch, 1.8.1894. INV.NO.1527 (1 F): Singa sanguinea C.L. Koch, Zadar, 25.7.1899. INV.NO.1532 (1 F): Singa sanguinea C.L. Koch, Bribir-Novi, 15.4.1897. INV. NO.1640 (1 M): Singa sanguinea C.L. Koch, Bakar. 
Larinioides cornutus (Clerck, 1757)

INV.NO.225 (1 JUV*): Epeira cornuta Clerck. INV.NO.226 (1 F, 1 JUV*): Epeira cornuta Clerck., Osijek. INV.NO.227 (1SSP*): Epeira cornuta Clerck., Vrana. INV.NO.445 (5 F, $1 \mathrm{M}, 1 \mathrm{JUV}^{*}$ ): Epeira cornuta Clerck., Zagreb. INV.NO.1596 (2 F, 1 M): Epeira Sturmii Hahn., Plitvice, 25.05.1902., Šnap. INV.NO.1644 (2 F): Epeira sclopetaria Clerck., Bakar. INV.NO.1661 (2SSP*): Epeira cornuta Clerck., Delnice. INV.NO.1677 (1 M, 4 SUBM $\left.{ }^{*}\right)$ : Epeira cornuta Clerck., Zemun. INV.NO.2150 (1 F): Aranea foliata, Kolansko blato, o. Pag, 4.10.1957. INV.NO.2392 (1 F): Tegenaria luxurians Kulcz., Osijek, 1897., Langhoffer.

Larinioides ixobolus (Thorell, , 1873)

INV.NO.321 (1 M): Epeira ixobola Thor., Križevci, Damin. INV.NO.321 (3 F): Epeira ixobola Thor., Križevci, Damin. INV.NO.446 (1 SUBM*): Epeira ixobola Thor., Zemun, Damin. INV.NO.446 (2 M): Epeira ixobola Thor., Zemun, Damin. INV.NO.446 (3 F): Epeira ixobola Thor., Zemun, Damin. INV.NO.446 (4 JUV*): Epeira ixobola Thor., Zemun, Damin. INV.NO.1513 (1 F): Epeira ixobola Thor., Križevci, 4.9.1897. INV.NO.1633 (1 JUV*): Epeira ixobola Thor., Osijek. INV.NO.1633 (1 F): Epeira ixobola Thor., Osijek. INV.NO.321 (1 $\mathrm{JUV}^{*}$ ): Epeira ixobola Thor., Križevci, Damin.

Larinioides patagiatus (Clerck, 1757)

INV.NO.444 (5 F, 1 M): Epeira patagiata Clerck., Damin. INV.NO.1361 (1 M): Tegenaria luxurians Kulcz., Osijek, 1897., Langhoffer. INV.NO.1361 (2 F): Tegenaria luxurians Kulcz., Osijek, 1897., Langhoffer. INV.NO.1511 (1 JUV*): Epeira patagiata Clerck., Zagreb, 1895. INV.NO.1515 (1 JUV*): Epeira patagiata Clerck., Osijek, 1907., Dr. Langhoffer. INV.NO.1518 (6 F, 2 SUBM$\left.^{*}, 1 \mathrm{JUV}^{*}\right)$ : Epeira patagiata Clerck. INV.NO.1524 (1 F): Epeira patagiata Clerck., Plitvice, 18.8.1898. INV.NO.1528 (1 F): Epeira patagiata Clerck., Plitvice, 27.8.1902. INV.NO.1533 (1 M): Epeira patagiata Clerck., Fužine, 06.06.1897., Dr. Langhoffer. INV. NO.1537 (1 JUV*): Epeira patagiata Clerck., Topusko, 08.1901. INV.NO.1537 (1 SUBM*): Epeira patagiata Clerck., Topusko, 08.1901. INV.NO.1656 (1 F, 1 M): Epeira patagiata Clerck., Zagreb.

Larinioides sp.

INV.NO.1663 (1 SUBM): Singa semiatra, Bažinec, 29.4.1911. INV.NO.2085\#: Epeira sp.

Larinioides suspicax (O. Pickard-Cambridge, 1876)

INV.NO.1663 (6 F): Singa semiatra, Bažinec, 29.4.1911. INV.NO.2103 (1 F): Aranea foliata, Kolani, 26.07.1956. INV.NO.2116 (1 F): Meta segmentata Clerck., Caska, o. Pag, 24.06.1960. INV.NO.2138 (1 F): Aranea foliata, Caska, Kolansko blato, 11.06.1958.

Leviellus sp.

INV.NO.1636 (2 JUV): Zilla Stroemii Thor., Ogulin. INV.NO.1645 (2 SSP, 5 JUV): Zilla Stroemii Thor., Zemun. INV.NO.1651 (11 JUV): Zilla Stroemii Thor., Topusko, 8.1890. INV. NO.1666 (1 SUBM): Zilla Stroemii Thor., Zagreb, 8.1912.

Leviellus thorelli (Ausserer, 1871)

INV.NO.118 (4 F): Zilla Stroemii Thor., Zemun, Damin. INV.NO.1645 (4 F): Zilla Stroemii Thor., Zemun.

Mangora acalypha (Walckenaer, 1802)

INV.NO.820 (11 F, 1 SUBF*, 2 JUV*$^{*}$ ): Mangora acalypha Walck., Bakar, Damin. INV. NO.821 (1 M): Mangora acalypha Walck., Budva, Damin. INV.NO.821 (1 F): Mangora acalypha Walck., Budva, Damin. INV.NO.822 (1 F, 1 JUV*): Mangora acalypha Walck., 
INV.NO.1119 (1 SUBM ${ }^{*}, 1$ JUV $^{*}$ ): Mangora acalypha Walck., Bribir-Novi, 15.4.1897. INV.NO.1152 (1 JUV*): Mangora acalypha Walck., Dol-Grižane, 14.4. INV.NO.1153 (2 F): Mangora acalypha Walck., Križevci, 14.4.1897. INV.NO.1157 (1 F, 1 JUV*): Mangora acalypha Walck. INV.NO.1160 (1 F): Mangora acalypha Walck., Ogulin, 4.6.1898. INV. NO.1648 (1 F): Zilla Keyserlingii Auss. Požega, 5.7.1905. INV.NO.1660 (1 F): Epeira patagiata Clerck., Obedska bara, 11.07.1913., Poljugan. INV.NO.2316 (1 F): Tarentula Apuliae Walck., Nad Bukom Krke, Damin.

\section{Neoscona adianta (Walckenaer, 1802)}

INV.NO.453 (11 F, 3 M): Epeira adianta Walck. INV.NO.2080 (4 F): Epeira adianta Walck., Kolansko blato, o. Pag, 27.6.1960. INV.NO.2088 (2 F): Epeira adianta Walck., Kolansko blato, o. Pag, 27.06.1960. INV.NO.2092 (1 F): Epeira adianta Walck., Caska, o. Pag, 12.06.1958. INV.NO.2104 (1 F): Epeira adianta Walck., o. Susak, 16.06.1962. INV.NO.2105 (1 F): Epeira adianta Walck., Kolani, 26.08.1956. INV.NO.2108 (2 F, 1 M): Epeira adianta Walck., Povljane, 01.07.1960. INV.NO.2126*(2 F): Epeira adianta Walck., St. Novalja, o. Pag, 24.06.1960. INV.NO.2127 (1 M): Epeira adianta Walck., Caska, 25.06.1960. INV.NO.2130 (3 F): Aranea adianta, Barbat, o. Pag, 22.06.1960. INV.NO.2134 (7 F): Epeira adianta Walck., Unije, 01.10.1963. INV.NO.2140 (1 F): Epeira adianta Walck., Kolansko blato, 11.06.1958. INV.NO.2158 (2 F): Epeira adianta Walck., Caska, o. Pag, 21.06.1960. INV.NO.2176 (16 F , 1 M, 1 SUBM $^{*}$ ): Epeira adianta Walck., Caska, o. Pag, 09.06.1958. INV.NO.2180 (2 F): Epeira adianta Walck., Caska, o. Pag, 28.06.1960. INV.NO.2184 (1 SUBF*): Epeira adianta Walck., Susak, 21.06.1962. INV.NO.2186 (1 F, 2 M): Epeira adianta Walck., Unije, 1-10.09.1963. INV. NO.2188 (5 F): Epeira adianta Walck., Caska, o. Pag, 26.06.1962. INV.NO.2200 (12 F): Epeira adianta Walck., Unije-Polje, 26.06.1963. INV.NO.2220\#: Aranea adianta. INV.NO.2222 (2 F): Epeira adianta Walck., Novi, 07.1918. INV.NO.2225 (3 F): Epeira adianta WalckKotor, 01.07.1931. INV.NO.2450 (3 F): Epeira adianta Walck., Barbat, o. Pag, 22.06.1960. INV. NO.453 (1 SUBM*, 6JUV*): Epeira adianta Walck. INV.NO.453 (): Epeira adianta Walck.,

\section{Neoscona sp.}

INV.NO.2446 (2 JUV): Epeira angulata Clerck, Kotor, 01.07.1931.

\section{Neoscona fusca (C. L. Koch, 1837)}

INV.NO.433 (1 F, 4SUBF*, 1 JUV*): Epeira dalmatica Dol., Omiš. INV.NO.434 (1 F, $1 \mathrm{M}$, 2 JUV $^{*}$ ): Epeira dalmatica Dol. INV.NO.1456 (1 SUBF*): Epeira dalmatica Dol., Bribir-Novi, 15.04.1897. INV.NO.1462 (1 F): Epeira dalmatica DOI., Zadar, 25.07.1899. INV.NO.1468 (1SSP*): Epeira dalmatica Dol., Novi, 25.09.1901. INV.NO.1634 (2 M): Epeira dalmatica, Jablanac. INV.NO.1641 (3 M): Epeira dalmatica Dol., Jablanac. INV.NO.1642 (1 F): Epeira dalmatica Dol., Kotor, 03.08.1894. INV.NO.1650 (3 F): Epeira juniperi Emert.H.C.Mc.Cook, 3. INV.NO.2093 (1 F): Epeira sp., Caska, o. Pag, 12.06.1938. INV.NO.2094 (1 F): Epeira adianta Walck., Novalja, Kolansko blato, 01.07.1955. INV.NO.2407 (1 F): Epeira Redii Scop., Primorje.

\section{Nuctenea umbratica (Clerck, 1757)}

INV.NO.228 (2 F): Epeira umbratica Clerck., Zemun, Damin. INV.NO.229 (5 F, 14 JUV*): Epeira umbratica Clerck., Bakar, Damin. INV.NO.440 (1 M): Epeira umbratica Clerck., Bakar (strop), 21.07.1899., Damin. INV.NO.1489 (2 JUV*): Epeira umbratica Clerck., Topusko, 8.1891. INV.NO.1520 (2 F): Epeira umbratica Clerck., Ogulin, 19.6.1896. INV. NO.1521 (1 F): Epeira umbratica Clerck., INV.NO.1529 (1 M): Epeira umbratica Clerck., Topusko, 8.1891. INV.NO.1639 (1 F): Epeira umbratica Clerck., Ogulin. INV.NO.1828 $\left(1 \mathrm{JUV}^{*}\right)$ : Epeira umbratica Clerck., Križevci. INV.NO.1828 (1 F, 4M): Epeira umbratica Clerck., Križevci. 
Singa hamata (Clerck, 1757)

INV.NO.659 (3 F, 1 SUBM*, 1 JUV*): Singa hamata Clerck., Božjakovina, Langhoffer. INV.NO.659 (): Singa hamata Clerck., Božjakovina, Langhoffer. INV.NO.660 (2 M): Singa hamata Clerck., Božjakovina, Langhoffer. INV.NO.661 (2 F, 3 M, 6 SUBM*, 7 JUV*): Singa hamata Clerck., Osijek. Osijek. INV.NO.1492 (2 M): Singa nitidula C.L. Koch, Osijek, 1897., Langhoffer.

Singa nitidula C. L. Koch, 1844

INV.NO.662 (1 SUBM*, 3 JUV*$^{*}$ ): Singa nitidula C.L. Koch, Damin. INV.NO.665 (1 F): Singa sanguinea C.L. Koch, Damin. INV.NO.1486 (1 F): Singa nitidula C.L. Koch, Križevci, 7.9.1897. INV.NO.1516 (1 SUBM*): Singa nitidula C.L. Koch, Osijek, 19.4.1899. INV.NO.1700 (1 JUV*): Singa nitidula C.L. Koch, Zemun. INV.NO.1854 (4 F, 2 M, 1 JUV*): Singa Herii Hahn., Trapsa?, 07.08.1903.

Singa sp.

INV.NO.1654 (1 SUBM, 3 JUV): Zilla Thorell, ii Auss. Babina greda, 17.7.1918. INV. NO.2398 (1 JUV*): Epeira Sturmii Hahn., Osijek, 1897., Dr. Langhoffer. INV.NO.2419 (1 JUV): Dolomedes fimbriatus Clerck., Unčica, 29.08.1911.

Stroemiellus stroemi (Thorell, , 1870)

INV.NO.118 (3 JUV*): Zilla Stroemii Thor., Zemun, Damin. INV.NO.119 (2 F, 1 M, 1 $\mathrm{JUV}^{*}$ ): Zilla Stroemii Thor., Osijek, Damin. INV.NO.140 (1 F): Zilla Thorell, ii Auss. Ogulin, 19.06.1896., Damin.

Zilla diodia (Walckenaer, 1802)

INV.NO.447 (1 JUV*): Epeira diodia Walck., Budva, Damin. INV.NO.448 (19 F, 2 M, 3 SUBF$^{*}, 1$ SUBM* ${ }^{*} 1$ JUV$^{*}$ ): Epeira diodia Walck., Bakar, Damin. INV.NO.1439 (1 F): Epeira diodia Walck., Bribir-Novi, 14.4.1897. INV.NO.1449 (1 F): Epeira diodia Walck., Zagreb, 9.7.1902. INV.NO.1483 (2 F): Epeira diodia Walck., Bribir-Novi, 15.4.1897.

Zygiella atrica (C. L. Koch, 1845)*

INV.NO.1496 (1 F): Zilla Keyserlingii Auss. Bakar (Platan), 1.3. INV.NO.2259 (3 F): Zilla Keyserlingii Auss.

Zygiella keyserlingi (Ausserer, 1871)

INV.NO.133 (2 M): Zilla Keyserlingii Auss. INV.NO.1487 (1 M): Zilla Keyserlingii Auss., Rijeka, 23.9.1987. INV.NO.1488 (1 SUBM*): Zilla Keyserlingii Auss., Križišće, 6.8.1893. INV. NO.1493 (2 JUV*): Zilla Keyserlingii Auss., Lukovo, 19.7.1899. INV.NO.2121 (6 F): Zilla litterata Olv., Caska, o. Pag, 24.06.1960. INV.NO.130 (1SSP*): Zilla Keyserlingii Auss., Skradin, Damin. INV.NO.131 (1 JUV*): Zilla Keyserlingii Auss., Starigrad, Damin. INV. NO.132 (2 JUV*): Zilla Keyserlingii Auss., Budva, Damin.

Zygiella sp.

INV.NO.1347 (1 JUV): Zodarium gallicum E. Sim., Trsat, 17.9. INV.NO.1494 (1 SUBM, 4 JUV): Zilla sp. INV.NO.1525 (1SSP*): Zilla sp., Starigrad, 11.7.1899. INV.NO.1530 (3 JUV): Zilla sp., Bag, 14.7.1899. INV.NO.1535 (1 SUBM, 1 JUV): Zilla sp. Vrata, 22.7. INV.NO.1536 (2 SUBM, 2 JUV): Zilla sp., Crikvenica, 08-12.08. INV.NO.1539 (6JUV): Zilla sp., Borova draga, 8.7.1897. INV.NO.1540 (1 JUV): Zilla sp., Skradin, 23.7.1892. INV.NO.1632 (5 JUV): Zilla atrica C.L. Koch, Jablanac, 11.07.1899. INV.NO.2390 (1 JUV): Zodarium elegans E. Sim., Bag, 7.9.1899. INV.NO.2403 (1 JUV): Epeira sp., Sv. Kuzam, 20.8.1902., Polić. INV. NO.2437 (1 JUV): Theridium aulicum C.L. Koch, Cavtat, 12.1898. 
Zygiella $x$-notata (Clerck, 1757)

INV.NO.134 (8 F): Zilla litterata Olv., 03.1890., Damin. INV.NO.135 (1 F): Zilla litterata Olv., Vis, Damin. INV.NO.136 (4 F): Zilla litterata Olv., Lokve, 1899., Damin. INV.NO.138 (2 F): Zilla litterata Olv., Martinšćica, Damin. INV.NO.664 (1 F): Singa sanguinea C.L. Koch. INV.NO.2039 (7 F): Zilla litterata Olv. INV.NO.2096 (2 F): Zilla sp., o. Susak, 16.06.1962. INV.NO.134 (1 SUBM*, 4 JUV*): Zilla litterata Olv., 03.1890., Damin. INV.NO.135 (1 JUV*): Zilla litterata Olv., Vis, Damin. INV.NO.136 (1 SUBM $\left.{ }^{*}\right)$ : Zilla litterata Olv., Lokve, 1899., Damin. INV.NO.137 (2SUBM*, 1 JUV $^{*}$ ): Zilla litterata Olv., Kotor, Damin. INV.NO.139 (1 $\left.\mathrm{SUBM}^{*}, 1 \mathrm{JUV}^{*}\right)$ : Zilla litterata Olv.

\section{Clubionidae Wagner, 1887}

Clubiona alpicola Kulczyński, 1882

INV.NO.1731 (2 F): Clubiona alpicola Kulcz., Tatra.

Clubiona brevipes Blackwall, 1841

INV.NO.355 (1 JUV*): Clubiona brevipes BI., Košljun, Damin. INV.NO.1364 (1 M): Clubiona brevipes BI., Fužine, 6.6.1897. INV.NO.1567 (1 F): Clubiona brevipes BI., Žakalj, 17.9. INV.NO.1862 (2 F, 1 M): Clubiona brevipes, Rijeka.

Clubiona comta C. L. Koch, 1839

INV.NO.354 (1 M, 1 SSP*): Clubiona compta C.L. Koch, Bakar (na bršljenu), 17.04.1891., Damin. INV.NO.357 (6 F, 1 SUBM*): Clubiona compta C.L. Koch, Batulj, 22.5.1890., Damin. INV.NO.1370 (2 JUV*): Clubiona compta C.L. Koch, Topusko, 08.1891. INV.NO.1415 (6JUV*): Clubiona compta C.L. Koch, Bitoraj (podnožje), 26.6.1891. INV.NO.2046 (2 JUV*): Clubiona compta C.L. Koch.

Clubiona corticalis (Walckenaer, 1802)***

INV.NO.358 (2 JUV*): Clubiona corticalis Walck., Crni lug, Damin.

Clubiona decora Blackwall, 1859***

INV.NO.356 (1 JUV*): Clubiona decora BI., Jablanac, 11.07., Damin. INV.NO.1381 (2 $\left.\mathrm{JUV}^{*}\right)$ : Clubiona decora Bl., Bakar, 1.3.

Clubiona frutetorum L. Koch, $1867^{* * *}$

INV.NO.1878 (8SUBF*): Clubiona frutetorum L. Koch, Osijek, 13.01.1898., Langhoffer. INV.NO.1882 (1 SUBF*, 2SUBM*): Clubiona frutetorum L. Koch, Osijek, 13.01.1898., Langhoffer. INV.NO.2059 (1 SUBM*): Clubiona frutetorum L. Koch. INV.NO.2059 (1 $\left.\mathrm{SUBF}^{*}\right)$ : Clubiona frutetorum L. Koch.

Clubiona genevensis L. Koch, 1866

INV.NO.353 (1 F): Clubiona decora BI., Sv.Duh, o.Krk, Damin. INV.NO.1897 (1 F): Clubiona decora BI., Risnjak.

Clubiona germanica Thorell, , 1871

INV.NO.362 (1 M): Clubiona germanica Thorell,. INV.NO.362 (3 F): Clubiona germanica Thorell,. INV.NO.1386 (1 F): Clubiona germanica Thorell,. INV.NO.1388 (1 F): Clubiona germanica Thorell, Bosna, Apfelbeck. 
Clubiona leucaspis Simon, 1932

INV.NO.1376 (1 F, 1 M): Clubiona decora BI.

Clubiona lutescens Westring, 1851

INV.NO.1707 (2 F, 1 M): Clubiona lutescens Westr., Galicija.

Clubiona pallidula (Clerck, 1757)

INV.NO.359 (2 F, 1 SUBF*): Clubiona pallidula CI., Osijek, Damin. INV.NO.1715 (1 M): Clubiona pallidula CI., Osijek. INV.NO.1715 (1 SUBM*): Clubiona pallidula CI., Osijek. INV.NO.1876 (1 SUBF*): Clubiona pallidula Cl., Plitvice, 26.08.1902. INV.NO.1880 (1 M): Clubiona pallidula CI., Ogulin.

Clubiona phragmitis C. L. Koch, 1843

INV.NO.364 (1 F): Clubiona phragmitis C.L. Koch, Buk Krke, Damin.

Clubiona reclusa O. P.-Cambridge, 1863

INV.NO.1872 (1 F): Clubiona reclusa Cambr.

Clubiona similis L. Koch, 1867

INV.NO.360 (1 F, 1 M): Clubiona similis L. Koch, Mrzla vodica, Damin.

Clubiona sp.

INV.NO.1391 (1 SUBF): Clubiona sp., Osijek, 06.0897., Dr. Langhoffer. INV.NO.1393 (2 JUV): Clubiona sp., Zagreb, 1901., Z. Bučan. INV.NO.1405 (15 JUV): Clubiona sp., Topusko, 8.1891. INV.NO.2385 (1 SUBM): Scytodes thoracica Latr., Kostrena Sv. Barbara, 2.5.1890.

Clubiona subsultans Thorell, 1875

INV.NO.1702 (2 F, 1 M): Clubiona subsultanus Thorell,.

Clubiona terrestris Westring, 1851

INV.NO.361 (1 F, 1 M): Clubiona terrestris Westr., Vrata, Damin. INV.NO.363 (1 F): Clubiona subtilis L. Koch, Zec, Damin. INV.NO.1410 (1 M): Clubiona terrestris Westr., Rječina, 22.5. INV.NO.1870 (1 M): Clubiona pallidula CI., Jelenje, 02.07.1893.

Clubiona trivialis C. L. Koch, 1843

INV.NO.365 (2 F, 2 M): Clubiona trivialis C.L. Koch, Rijeka, Damin. INV.NO.1421 (1 M): Clubiona trivialis C.L. Koch, Bitoraj, podnožje, 26.6.1892. INV.NO.1421 (1 F): Clubiona trivialis C.L. Koch, Bitoraj, podnožje, 26.6.1892. INV.NO.1697 (2 F): Clubiona trivialis C.L. Koch, Mrzla vodica.

\section{Corinnidae Karsch, 1880}

Corinnidae gen. sp.

INV.NO.370 (4SSP): Trachelas maculatus Thorell, Jablanac.

\section{Cybaeidae Banks,1892}

Cybaeus sp. INV.NO.1352 (1SSP*): Cybaeus sp.

INV.NO.1353 (2SSP*): Cybaeus sp., Bitoraj-podnožje, 26.06.1892. 
Cybaeus tetricus (C. L. Koch, 1839)

INV.NO.1239 (2 F, 1 M): Dipoena melanogaster C.L. Koch, Zagreb-Sofijin put, 19.06.1898. INV.NO.2014\#: Cybaeus tetricus C.L. Koch, Bakar (škver), 09.05.1890. INV. NO.1357\#: Cybaeus tetricus C.L. Koch, 1902., Broz. INV.NO.1358\#: Cybaeus tetricus C.L. Koch, 12.07.1902., Siničić.

\section{Dictynidae O. Pickard-Cambridge, 1871}

Brigittea civica (Lucas, 1850)

INV.NO.124 (11 F, 4SUBF*, 4 SUBM*, 3 M): Dictyna civica Lucas, Novigrad-Zadar, Damin N. INV.NO.126 (3 SUBM ${ }^{*}$ ): Dictyna civica Lucas, 20.06.1890., Damin N. INV. NO.126 (4 F): Dictyna civica Lucas, 20.06.1890., Damin N. INV.NO.126 (9M): Dictyna civica Lucas, 20.06.1890., Damin N. INV.NO.2244 (1 F): Emblyna sp., Bakar, Damin N.

Brigittea latens (Fabricius, 1775)

INV.NO.10 (2 F): Dictyna latens Fabr., Omišalj, Damin N. INV.NO.171 (2 F): Dictyna latens Fabr., Bakar, 7.8.1889., Damin N. INV.NO.172 (2 F): Dictyna latens Fabr., Solin, Damin N. INV.NO.2005 (2 F): Dictyna latens Fabr., Omišalj.

Dictyna arundinacea (Linnaeus, 1758)

INV.NO.127 (1 SUBM*): Dictyna arundinacea L., Zagreb, Damin N. INV.NO.129 (6 SUBF$^{*}, 2$ SUBM*): Dictyna arundinacea L., Osijek, Damin N. INV.NO.1170 (2 F): Dictyna arundinacea L. Koch, Osijek, 7.1901.

Dictyna uncinata Thorell, , 1856

INV.NO.128 (6 F, 2 SSP*, 6 M): Dictyna uncinata Thor., Ogulin, Damin N. INV.NO.1194 (2 F): Dictyna uncinata Thor., Ogulin, 19.4.1896. INV.NO.1198 (3 JUV*): Dictyna uncinata Thor. INV.NO.2011 (1 F, 1 M): Dictyna uncinata Thor., Zemun, 1899.

Dictynidae gen. sp.

INV.NO.1189 (1 JUV): Lathys humilis Blackw., Mali Grad.

Lathys humilis (Blackwall, 1855)

INV.NO.19 (27 F, 5 SUBM*, 1 JUV*): Lathys humilis Blackw., Bakar, Damin N. INV. NO.20 (1 F): Lathys humilis Blackw., Košljun (Krk), Damin N. INV.NO.1185 (1 F): Lathys humilis Blackw., Bribir-Novi, 15.6.1897. INV.NO.2311 (1 F): Micrommata virescens Clerck. INV.NO.2312 (1 F): Filistata insidiatrix Forsk., Vis, Damin N.

Nigma flavescens (Walckenaer, 1830)

INV.NO.176 (1 F, $1 \mathrm{JUV}^{*}$ ): Dictyna flavescens Walck., Ratulje, 22.5., Damin N. INV. NO.177 (1 SUBM ${ }^{*}$ ): Dictyna flavescens Walck., Vis, 11.04.1889., Damin N. INV.NO.178 (4 F): Dictyna flavescens Walck., Bakar (grm), 23.05.1890., Damin N. INV.NO.2018 (1 F): Theridium sp., Osijek, 25.09.1917.

Nigma walckenaeri (Roewer, 1951)

INV.NO.125 (7 F, 4 M): Dictyna vicina E. Simon, Osijek, Damin N. INV.NO.200 (2 JUV*):

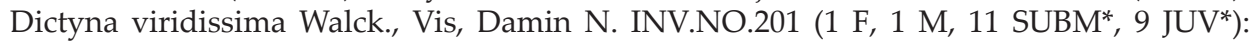
Dictyna viridissima Walck., Bakar, Damin N. INV.NO.202 (1 F): Dictyna viridissima Walck., Pecina (Vrana), 23.7., Damin N. INV.NO.1174 (2 JUV*): Dictyna viridissima Walck., Stari grad, 29.07., Damin N. INV.NO.1179 (3 JUV*): Dictyna viridissima Walck., Bag, 14.07.1898., 
Damin N. INV.NO.1184 (1SSP*): Dictyna viridissima Walck., SI. Pazova, 10.08.1898., Damin N. INV.NO.2264 (2 F, 1 M): Dictyna flavescens Walck., Bakar (grm), 23.05.1890., Damin N.

\section{Dysderidae C. L. Koch, 1837}

Dasumia canestrinii (L. Koch, 1876)

INV.NO.457 (1 SUBM*): Harpactes Canestrinii L. Koch, Bakar, 28.08.1889., Damin N. INV.NO.1416 (1 M): Harpactes Canestrinii L. Koch, Žakalj, 27.9. INV.NO.1423 (1 F): Harpactes Canestrinii L. Koch, Malinska, 27.3. INV.NO.1426 (1 F): Harpactes Canestrinii L. Koch, Bakar.

Dasumia chyzeri (Kulczyński, 1906)

INV.NO.272 (1 F): Dysdera erythrina Latr., Gasperini.

Dysdera adriatica Kulczyński, 1897

INV.NO.1717 (1 M): Dysdera hungarica v. adriatica Kulczinsky, Bosna, Apfelbeck.

Dysdera cf. granulata Kulczyński, 1897

INV.NO.271 (1 F): Dysdera erythrina Latr., Palagruža, 26.05.1911., Dr. K. Babić. INV. NO.2282 (1 F): Dysdera Ninnii Can.-Pav., Gasperini. INV.NO.2285 (2 F, 2 M): Dysdera Ninnii Can.-Pav.Bakar, Damin N.

Dysdera crocata C. L. Koch, 1838

INV.NO.270 (11 F, 7 M): Dysdera crocota C.L. Koch, Gasperini. INV.NO.274 (1 F): Dysdera erythrina Latr., Split, Damin N. INV.NO.275 (4 F, 1 JUV*): Dysdera crocota C.L. Koch, Trogir, Damin N. INV.NO.278 (4 F, 1 M): Dysdera crocota C.L. Koch, Senj, Damin N. INV.NO.1935 (1 M): Dysdera crocota C.L. Koch, Sunto, 17.03. INV.NO.1935 (1 F): Dysdera crocota C.L. Koch, Sunto, 17.03. INV.NO.2279 (1 F, 1 M): Dysdera erythrina Latr., Gasperini.

Dysdera dubrovninnii Deeleman-Reinhold, 1988

INV.NO.276 (1 F, 2 M): Dysdera punctata C. Koch, Gasperini. INV.NO.280 (1 F): Dysdera punctata C. Koch, Ombla (Rijeka), Damin N. INV.NO.1945 (1 F): Dysdera crocota C.L. Koch, Zlobin-Lič, 02.09.1915. INV.NO.2281 (4 F): Dysdera Ninnii Can.-Pav., Gasperini. INV.NO.2284 (1 F, 2 M): Dysdera punctata C. Koch, Bakar, Damin N. INV.NO.2435 (1 F): Dysdera Ninnii Can.-Pav.Bakar.

Dysdera ninnii Canestrini, 1868

INV.NO.8 (1 F, 1 M): Ballas depressus Walck., Gasperini. INV.NO.273 (1 F, $1 \mathrm{M})$ : Dysdera erythrina Latr., Bakar, 20.09., Damin N. INV.NO.277 (1 M): Dysdera Ninnii Can.Pav., Gasperini. INV.NO.279 (2 F, 3 M): Dysdera Ninnii Can.-Pav., Jablanac, Damin N. INV.NO.282 (5 F, 3 M): Dysdera Ninnii Can.-Pav., Bakar, Damin N. INV.NO.534 (1 F): Dysdera Ninnii Can.-Pav., Malinska, 27.3. INV.NO.1163 (1 SUBF*): Pachygnatha Degeerii Sund., Osijek, 4.1899. INV.NO.1401 (1 M): Dysdera Ninnii Can.-Pav., Kostrena Sv. Lucija. INV.NO.1412 (1 F): Dysdera Ninnii Can.-Pav., Bosna, Apfelbeck. INV.NO.1432 (1 JUV*): Dysdera Ninnii Can.-Pav., Žakalj, 17.9. INV.NO.1576 (1 M): Drassodes minusculus (=Dalmat) L. Koch, Senj. INV.NO.1940 (1 JUV*): Dysdera Ninnii Can.-Pav., Jablanac, 11.07.1899. INV.NO.1941 (1 F): Dysdera bocota C.L. Koch, Nad Ponikvama. INV.NO.1942 (1 F): Dysdera Ninnii Can.-Pav., Bakar. INV.NO.1943 (3 F): Dysdera Ninnii Can.-Pav., Risnjak, 19.08. INV.NO.2280 (1 M): Dysdera punctata C. Koch, Gasperini. INV.NO.2283 (2 F): Dysdera punctata C. Koch, Bakar, Damin N. INV.NO.2434 (1 M): Dysdera crocota C.L. Koch, Sunto, 17.03. 
Dysdera punctata C. L. Koch, $1838^{* * *}$

INV.NO.281 (1 JUV*): Dysdera punctata C. Koch, Bakar, Damin N.

Dysdera sp.

INV.NO.1400 (2 JUV): Dysdera sp., Risnjak, 4.7. INV.NO.1406 (1 JUV): Dysdera sp., Bag, 14.7.1899. INV.NO.1408 (3 JUV): Dysdera sp. INV.NO.2223\#: Dysdera sp., Kotor, 01.07.1931.

Dysderocrates storkani (Kratochvíl, 1935)

INV.NO.458 (1 F): Harpactes rubicundus C.L. Koch, Nad Senjom, Damin N.

\section{Eresidae C. L. Koch, 1845}

Eresus kollari Rossi, 1846

INV.NO.911 (1 SUBF*): Eresus niger Petagna., Karlobag, 13.07.1899., Damin N. INV. NO.912 (1 SUBF*, 3 JUV*): Eresus niger Petagna., Bakar, Damin N. INV.NO.913 (1 SUBF*, $1 \mathrm{M}$ ): Eresus niger Petagna., Senj, Damin N. INV.NO.914 (1 M): Eresus niger Petagna., Sv. Filip i Jakov (Dalm.), Damin N. INV.NO.915 (1 M): Eresus niger Petagna., Bakar, 11.9.1900., Damin N. INV.NO.916 (35 JUV*): Eresus niger Petagna., Damin N. INV.NO.2119 (1 F): Eresus niger Petagna., Poljane, 01.07.1960. INV.NO.2148 (1 M): Eresus niger Petagna., Unije, Poljane, 10.09.1963.

\section{Cheiracanthiidae Wagner, 1887}

Cheiracanthium elegans Thorell, $1875^{* * *}$

INV.NO.1425 (1 SUBF*): Chiracanthium Letochae, Kraljičin zdenac, 14.06.1897., Dr. Langhoffer.

Cheiracanthium mildei L. Koch, 1864

INV.NO.399 (1 M, 1 JUV*): Chiracanthium Mildei L. Koch. INV.NO.399 (): Chiracanthium Mildei L. Koch. INV.NO.468 (1 F, 2 M, 1SUBF* 1 JUV*): Chiracanthium Mildei L. Koch, Senj, Damin. INV.NO.474 (1 M): Chiracanthium Mildei L. Koch, 17.05., Gasperini. INV.NO.474 (3 F): Chiracanthium Mildei L. Koch, 17.05., Gasperini. INV.NO.1430 (1 M): Chiracanthium Mildei L. Koch, Bakar, 24.07. INV.NO.1435 (1 F): Chiracanthium Mildei L. Koch, Bakar, 26.06. INV.NO.1690 (1 M): Chiracanthium Mildei L. Koch, Bakar, 06.03. INV.NO.1871 (1 M): Chiracanthium Mildei L. Koch, Martinšćica. INV.NO.1871 (1 F): Chiracanthium Mildei L. Koch, Martinšćica. INV.NO.1879 (1 M): Chiracanthium elegans Thorell, Ušće Raše, 31.05.1896. INV.NO.1888 (1 F): Chiracanthium sp., Borova draga (Sv. Juraj). INV.NO.1889 (2 F, 3 JUV*): Chiracanthium Mildei L. Koch, Stari grad (Dalmacija). INV.NO.2052 (2 F, 1 M): Chiracanthium Mildei L. Koch. INV.NO.2297 (1 F): Tegenaria Derhamii Scop., Senj, Damin. INV.NO.2298 (5 F, 2 M): Chiracanthium pelasgicum C.L. Koch, Zemun, Damin.

Cheiracanthium pelasgicum (C. L. Koch, 1837)

INV.NO.395 (2 M): Chiracanthium pelasgicum C.L. Koch, Hvar, Damin. INV. NO.396 (3 M): Chiracanthium pelasgicum C.L. Koch, Zadar, Damin. INV.NO.473 (2 M): Chiracanthium pelasgicum C.L. Koch, Gasperini. INV.NO.1875 (1 M): Chiracanthium sp., Solin, 26.07. INV.NO.1875 (1 F): Chiracanthium sp., Solin, 26.07. INV.NO.1895 (3 M): Chiracanthium pelasgicum C.L. Koch, Omišalj. INV.NO.1896 (1 F, 1 M): Chiracanthium pelasgicum C.L. Koch, Rijeka. INV.NO.2299 (2 JUV*): Chiracanthium pelasgicum C.L. Koch, Zemun, Damin. 
Cheiracanthium pennyi O. P.-Cambridge, 1873

INV.NO.397 (1 M): Chiracanthium pelasgicum C.L. Koch, Zemun, Damin.

Cheiracanthium punctorium (Villers, 1789)*

INV.NO.173 (1 F): Chiracanthium punctorium Villers., Bakar, 7.8., Damin. INV. NO.1562 (1 F): Chiracanthium punctorium Villers. INV.NO.1873 (4 SUBF*): Chiracanthium punctorium Villers., Šamarica (Dodoši), 02.08.1916. INV.NO.1892 (1 SUBF*): Chiracanthium punctorium Villers., Bakar, 26.07.1905. INV.NO.2044 (1 M, $\left.1 \mathrm{JUV}^{*}\right)$ : Chiracanthium punctorium Villers.

Cheiracanthium seidlitzi L. Koch, $1864^{* * *}$

INV.NO.398 (1 SUBM*): Chiracanthium Seidlitzii L. Koch, Gasperini.

Cheiracanthium sp.

INV.NO.1404 (2 JUV): Chiracanthium sp. INV.NO.1550 (1 JUV): Chiracanthium sp., Baška, 08.06.1897. INV.NO.2315 (1 JUV): Euryops acuminata Luc., Bakar, 4.8., Damin N. INV.NO.2368 (1 JUV): Philodromus lividus Sim., INV.NO.2380 (1 SUBM*): Philodromus aureolus Clerck verus, Prevez, Raša, Istra, Damin. INV.NO.2426 (1 SUBM): Clubiona sp., Bakar.

\section{Filistatidae Ausserer, 1867}

Filistata insidiatrix (Forsskål, 1775)

INV.NO.513 (3 F, 1 JUV*): Filistata insidiatrix Forsk., Dubrovnik, Damin N. INV.NO.514 (1 F): Filistata insidiatrix Forsk., 1893., Damin N. INV.NO.515 (3 F, 1 M, 1 JUV*): Filistata insidiatrix Forsk., Vis, Damin N. INV.NO.516 (3 JUV*): Filistata insidiatrix Forsk., Split, Damin N. INV.NO.1452 (1 JUV*): Filistata insidiatrix Forsk., Skradin, 23.7.1894. INV. NO.1460 (3 JUV*): Filistata insidiatrix Forsk., Stari grad. INV.NO.1484 (1 F): Filistata insidiatrix Forsk., Lukovo, 9.7.1899. INV.NO.1719 (2 JUV*): Filistata insidiatrix Forsk., Voz, 07.07.1891. INV.NO.1719 (2 F): Filistata insidiatrix Forsk., Voz, 07.07.1891. INV.NO.2250 (1 F): Enoplognatha mandibularis Luc., Gasperini. INV.NO.166 (19JUV*): Filistata testacea.

Filistata sp.

INV.NO.2141 (1 SUBF): Filistata sp., Unije, 25.06.1963. INV.NO.2250 (2 JUV): Enoplognatha mandibularis Luc., Gasperini.

Pritha nana (Simon, 1868)

INV.NO.30 (2 F, 1 M, 1 JUV*): Filistata nana E. Simon, Senj, 04.07., Damin N. INV.NO.31 (3 F, 1 M, 5 JUV $^{*}$ ): Filistata nana E. Simon, Bakar, 04.05.1890., Damin N. INV.NO.1441 (1 F): Filistata nana E. Sim., Lukovo, 9.7.1899. INV.NO.1465 (5 JUV*): Filistata nana E. Sim., INV. NO.1471 (1 F): Filistata nana E. Sim., Kraljevica, 31.07.1899. INV.NO.1478 (2 JUV*): Filistata nana E. Sim., Sv. Juraj, 17.7.

\section{Gnaphosidae Pocock, 1898}

Aphantaulax cincta (L. Koch, 1866)

INV.NO.145 (2 M): Aphantaulax cinctus L. Koch. Bakar, Damin. INV.NO.842 (1 M): Linyphia frutetorum C.L. Koch, Gasperini. INV.NO.1241 (1 F): Prosthesima subterranea C.L. Koch, Gasperini. 
Aphantaulax trifasciata (O. Pickard-Cambridge, 1872)\#

INV.NO.144\#: Aphantaulax Albinii (Sav.), Kotor, 03.08., Damin. INV.NO.257\#: Aphantaulax Albinii (Sav.), Solin. INV.NO.462\#: Aphantaulax Albinii (Sav.), Bakar, Košljun. INV.NO.463\#: Aphantaulax Albinii (Sav.), Košljun (Krk).

Berinda sp. nov.*

INV.NO.2378 (1 M): Liocranum tenuissimum L. Koch, Bakar, Damin.

Callilepis schuszteri (Herman, 1879)

INV.NO.108 (1 M): Callilepis nocturna, Damin. INV.NO.109 (1 F, 2 M): Callilepis Schuszteri O.Herm., Bakar, Crikvenica, Damin. INV.NO.1390 (1 F): Callilepis Schuszteri O.Herm.

Civizelotes caucasius (L. Koch, 1866)

INV.NO.1243 (1 F): Prosthesima caucasia L. Koch, Omišalj, Damin N.

Drassodes cupreus (Blackwall, 1834)

INV.NO.492 (1 F): Scotophaeus quadripunctatus L. Koch, Bakar. INV.NO.503 (1 F): Tarentula sp., Snježnik, Risnjak, Lazac - 1069m, 28.6., Varićak. INV.NO.2422 (1 F): Clubiona sp., Bakar.

Drassodes lapidosus (Walckenaer, 1802)

INV.NO.163 (2SUBM*): Drassodes lapidicola Wk., Gasperini. INV.NO.471 (1 F): Drassodes lapidicola Wk., Brgut (Krk), Damin N. INV.NO.472 (1 F): Drassodes lapidicola Wk.Buk Krke, Damin N. INV.NO.476 (1 M, 2 JUV*): Drassodes lapidicola Wk.Pod Srebračem, 13.05.1890., Damin N. INV.NO.502 (1 F, 2 M): Drassodes lapidosus Wk. INV. NO.1367 (1 SUBF*, 2 JUV*): Drassodes lapidicola Wk. INV.NO.1372 (1 F): Drassodes lapidicola Wk., Kraljevica, 8.7. INV.NO.1378 (1 F): Drassodes lapidicola Wk., Lukovo, 9.7.1899. INV.NO.1383 (1 F): Drassodes lapidicola Wk., 1902., Grgić. INV.NO.1394 (2 M): Drassodes lapidicola Wk. INV.NO.1397 (1 JUV*): Drassodes lapidicola Wk. INV.NO.1554 (1 F): Theridium lineatum Clerck., INV.NO.1890 (2 JUV*): Drassodes lapidicola Wk., Bakar, 18.09., Damin. INV.NO.2197 (1 F): Drassodes lapidosus Wk., Caska, o. Pag, 10.07.1958. INV.NO.2423 (2 F, 1 M): Clubiona sp., Bakar. INV.NO.2447 (3 F): Drassodes lapidicola Wk., Pod Srebračem.

Drassodes lutescens (C. L. Koch, 1839)

INV.NO.346 (1 JUV*): Drassodes lutescens C.L. Koch. Kotor, Damin. INV.NO.470 (3 F, 1 $\left.\mathrm{M}^{*}\right)$ : Drassodes lutescens C.L. Koch, Gasperini. INV.NO.779 (1 JUV*): Drassodes lutescens C.L. Koch, Pelagosa, 05.1911., Dr. K. Babić. INV.NO.780 (2SUBM*): Drassodes lutescens C.L. Koch, Pelagosa, 26.05.1911., Dr. K. Babić. INV.NO.2309 (1 F): Drassodes lapidosus Wk. INV.NO.2310 (5 JUV*): Drassodes lapidosus Wk.

Drassodes sp.

INV.NO.1365 (11 JUV): Drassodes sp. INV.NO.1371 (2 JUV): Drassodes lapidicola Wk.

INV.NO.1392 (1 JUV): Drassodes sp., Bakar (škver). INV.NO.1867 (1 SUBF): Drassodes sp., Gasperini. INV.NO.1898 (13 JUV): Clubiona sp., Bakar.

Drassyllus praeficus (L. Koch, 1866)

INV.NO.777 (1 F): Prosthesima pusilla C.L. Koch. 
Gnaphosa alacris Simon, 1878

INV.NO.122\#: Gnaphosa Kulczynski Damin, Pad Krke, Damin N.

Gnaphosa bicolor (Hahn, 1833)

INV.NO.1868\#: Gnaphosa bicolor Hahn., Klanjec, 1904., Broz. INV.NO.1886\#: Gnaphosa bicolor Hahn., Kralji, 28.06.1912.

Gnaphosa dolosa Herman, 1879

INV.NO.1874 (2 F): Scotophaeus loricatus L. Koch, Bakar, 03.05.1901. INV.NO.2303 (2 F): Drassodes lapidicola Wk., Pod Srebračem, 13.05.1890., Damin N. INV.NO.1380\#: Gnaphosa adriatica Kulcz., Žakalj, 17.09.

Gnaphosa sp.

INV.NO.1385\#: Gnaphosa sp. INV.NO.1594\#: Gnaphosa sp. INV.NO.1874 (2 JUV*): Scotophaeus loricatus L. Koch, Bakar, 03.05.1901. INV.NO.2303 (3 JUV, 1 SUBM): Drassodes lapidicola Wk., Pod Srebračem, 13.05.1890., Damin N.

Gnaphosidae gen. sp.

INV.NO.2271 (1 SUBM): Epiblemum mutabile LucasBakar, Kraljevica, Damin. INV. NO.2294 (1 JUV*): Clubiona subtilis L. Koch, Zec, Damin.

Haplodrassus dalmatensis (L. Koch, 1866)

INV.NO.180 (1 F): Drassodes minusculus (=Dalmat) L. Koch., Gasperini.

Haplodrassus signifer (C. L. Koch, 1839)

INV.NO.252 (2 M): Drassodes troglodytes C.L. Koch, Split, Damin N. INV.NO.459 (1 F, 1 M, 1 SUBF$^{*}, 1$ SUBM ${ }^{*}$ ): Drassodes troglodytes C.L. Koch, Punat, o. Krk, Damin N. INV.NO.475 (1SSP*, 8 F, 5M, 1 SUBM*): Drassodes troglodytes C.L. Koch, Gasperini. INV. NO.1377 (3 F): Drassodes troglodytes C.L. Koch.

Haplodrassus sp.

INV.NO.2414 (3 JUV): Callilepis exornata C.L. Koch.

Kishidaia conspicua (L. Koch, 1866)

INV.NO.1729 (1 M): Poecilochroa conspicua L. Koch, Kraljičin zdenac, 14.06.1897., Langhoffer.

Leptodrassus femineus (Simon, 1873)

INV.NO.899 (1 F): Leptodrassus femineus E. Sim., Lukovo, 9.7., Damin N.

Micaria albovittata (Lucas, 1846)

INV.NO.2313 (1 SUBM*): Micaria Rogenhoferi O. Herm., Bakar, 30.4.1890., Damin.

Micaria coarctata (Lucas, 1846)

INV.NO.545 (1 JUV*): Micaria chalibea, Bag, 14.7. INV.NO.1684 (1 F): Micaria chalibea, Karlobag. INV.NO.2022 (1 F): Micaria chalybea, Bag, 14.07.1899.

Micaria dives (Lucas, 1846)

INV.NO.548 (1 M): Micaria formicaria Sund., Bakar, Damin. INV.NO.1434 (1 M): Micariolepis dives Luc., Omišalj, 7.6. 
Micaria formicaria (Sundevall, 1831)

INV.NO.548 (1 SSP*): Micaria formicaria Sund., Bakar, Damin.

Micaria pallipes (Lucas, 1846)

INV.NO.547 (2 F): Micaria Rogenhoferi O. Herm., Bakar, 30.4.1890., Damin.

Micaria pulicaria (Sundevall, 1831)***

INV.NO.546 (3 JUV*, 2 SUBM* $^{*}$ : Micaria pulicaria Sund.

Micaria sp.

INV.NO.1429 (1 JUV): Micaria sp., Žakalj, 17.9.

Nomisia aussereri (L. Koch, 1872)

INV.NO.213 (1 F): Callilepis Aussereri L. INV.NO.449 (1 F): Callilepis Aussereri L. Koch, Karlobag, Damin N. INV.NO.450 (1 F): Callilepis Aussereri L. Koch, Kraljevica, Damin N.

Nomisia exornata (C. L. Koch, 1839)

INV.NO.179 (4 F, 2 M): Callilepis exornata C.L. Koch, Gasperini. INV.NO.310 (2 F, 2SUBM*, 22 JUV$^{*}$ ): Callilepis exornata C.L. Koch, Vis, Damin N. INV.NO.320 (6 F, 1 M, 2 SUBM $^{*}$ ): Callilepis exornata C.L. Koch, Split, Solin, Damin N. INV.NO.1559 (1 JUV*): Callilepis exornata C.L. Koch, Žakalj. INV.NO.1559 (2 M): Callilepis exornata C.L. Koch, Žakalj. INV.NO.1566 (1 M): Callilepis exornata C.L. Koch, Omišalj. INV.NO.1571 (2 $\mathrm{JUV}^{*}$ ): Callilepis exornata C.L. Koch. INV.NO.1579 (1 F): Ergane arcuata Clerck., INV. NO.1583 (1 F): Callilepis exornata C.L. Koch, Lukovo, 09.07.1899. INV.NO.1722 (1 M*, 4 JUV*): Callilepis exornata C.L. Koch. INV.NO.1893 (1 F): Callilepis exornata C.L. Koch, Senj, 06.07.1894. INV.NO.1894 (6JUV*): Callilepis exornata C.L. Koch, Bakar. INV.NO.2266 $\left(16 \mathrm{JUV}^{*}\right)$ : Callilepis exornata C.L. Koch, Gasperini.

Nomisia recepta (Pavesi, 1880)

INV.NO.343 (2 M): Callilepis exornata C.L. Koch, Damin N. INV.NO.343 (2 F): Callilepis exornata C.L. Koch, Damin N. INV.NO.2265 (11 M): Callilepis exornata C.L. Koch, Gasperini. INV.NO.2265 (26 F): Callilepis exornata C.L. Koch, Gasperini.

Nomisia sp.

INV.NO.1395 (2 JUV): Callilepis sp., Grižane, 14.04.1905. INV.NO.1399 (3 JUV): Callilepis sp. INV.NO.1547 (1 JUV): Callilepis sp., Bag, 07.09.1899. INV.NO.2334 (1 JUV): Lycosa annulata Thor., Rijeka, Bakar, Crikvenica, Damin. INV.NO.2353 (1 JUV): Anyphaena accentuata Walck., Bakar, 12.04.1892., Damin.

Scotophaeus scutulatus (L. Koch, 1866)

INV.NO.198 (1 F): Drassodes scutulatus L. Koch. INV.NO.491 (1 F): Scotophaeus scutulatus L. Koch, Bakar. INV.NO.1387 (1 F): Scotophaeus scutulatus C.L. Koch, Topusko, 08.1901. INV.NO.1885 (1 M): Phoeocoedus braccatus L. Koch, Selce, 20.08.1911.

Scotophaeus sp.

INV.NO.1382 (1 JUV*): Scotophaeus sp., Lukovo, 09.07.1899.

Setaphis carmeli (O. Pickard-Cambridge, 1872)***

INV.NO.256 (1 SUBM*, 2 JUV*): Prosthesima Carmeli Cambr., Gasperini. INV.NO.1242 (2 SUBM ${ }^{*}$ ): Prosthesima Carmeli Cambr., Split, Damin N. 
Trachyzelotes adriaticus (Caporiacco, 1951)

INV.NO.1244 (1 M): Prosthesima secreta Thor., Omišalj, Damin N.

Trachyzelotes barbatus (L. Koch, 1866)

INV.NO.533 (1 F): Prosthesima barbata L. Koch, Gasperini. INV.NO.538 (1 F): Prosthesima barbata L. Koch, Omišalj, Damin N. INV.NO.1366 (1 SUBM*, 1 JUV*): Prosthesima barbata L. Koch, Malinska, 27.3.

Trachyzelotes lyonneti (Audouin, 1826)

INV.NO.537 (1 F): Prosthesima barbata L. Koch, Crikvenica, Damin N.

Trachyzelotes pedestris (C. L. Koch, 1837)

INV.NO.566 (1 SUBF*): Prosthesima pedestris C.L. Koch, Sv. Duh (Krk), Damin N. INV.NO.1384 (2 M): Prosthesima pedestris C.L. Koch. INV.NO.2028 (1 SUBM*, 1 JUV*): Prosthesima pedestris C.L. Koch, 19.04.

Trachyzelotes sp.

INV.NO.1389 (1 JUV): Prosthesima sp., Žakalj. INV.NO.1396 (1 JUV): Prosthesima sp. INV.NO.1398 (1 JUV): Prosthesima sp., Bakar, 19.4.1891. INV.NO.2306 (2SUBM*): Zoropsis ocreata, Damin N. INV.NO.2364 (1 SUBM): Tarentula pulverulenta v. aculeata CI., Risnjak, $1000-150 \mathrm{~m}$.

Urozelotes rusticus (L. Koch, 1872)

INV.NO.318 (1 F): Prosthesima rustica L. Koch, Bakar, 23.7., Damin N. INV.NO.1368 (1 F): Prosthesima rustica L. Koch. INV.NO.1884 (1 M): Drassodes pubescens Thor., Vis.

Zelotes femellus (L. Koch, 1866)

INV.NO.689 (1 F): Prosthesima femella L. Koch, Gasperini.

Zelotes hermani (Chyzer, 1897)

INV.NO.486 (1 F, 1 SUBM*, 4 JUV*): Prosthesima Hermani Chyzer., Bakar, Damin N. INV.NO.1379 (2 F): Prosthesima Hermani Chyzer.

Zelotes oblongus (C. L. Koch, 1833)

INV.NO.298 (1 F): Prosthesima subterranea C.L. Koch, Zadar, Damin N. INV.NO.634 (1 F): Prosthesima oblonga L.Koch, Bakar, Damin N. INV.NO.1565 (1 F): Prosthesima oblonga L. Koch, Grižane, 14.9.1891.

Zelotes sp.

INV.NO.2267 (1 JUV): Callilepis exornata C.L. Koch, Gasperini.

Zelotes tenuis (L. Koch, 1866)

INV.NO.409 (1 F): Prosthesima circumspecta Sim., Bag, 14.7.1899., Damin N. INV. NO.1373 (1 F): Prosthesima circumspecta Sim., Bakar, 22.07.

\section{Hahniidae Bertkau, 1878}

Cryphoeca silvicola (C. L. Koch, 1834)

INV.NO.1363 (1 F): Cryphoeca sylvicola C.L. Koch, Bitoraj-podnožje, 26.6.1892. 
Hahnia nava (Blackwall, 1841)

INV.NO.154 (1 F): Hahnia nava Bl., Trsat, 16.3.1886., Damin.

\section{Linyphiidae Blackwall, 1859}

Agyneta rurestris (C. L. Koch, 1836)

INV.NO.1294\#: Micryphantes rurestis L., Koch, Križišće, 06.08.1893. INV.NO.1300*(2 F): Micryphantes rurestis L. Koch. INV.NO.1310 (1 F): Micryphantes rurestis L. Koch, Kecskemet, 1890. INV.NO.2247 (1 F): Trichoncus scrofa E. Sim., Crikvenica, 25.02., Damin.

Canariphantes nanus (Kulczyński, 1898)

INV.NO.1295 (1 F): Lephthyphantes nanus? Kecskemet, 1890.

Cresmatoneta mutinensis (Canestrini, 1868)

INV.NO.577 (9 F, 3 M, 3 SUBM*): Formicina mutinensis Canestr., Bakar, 19.09.1890., Damin. INV.NO.578 (1 M): Formicina mutinensis Canestr., Kotor, Damin. INV.NO.578 (1 F): Formicina mutinensis Canestr., Kotor, Damin. INV.NO.579 (1 F, 1 M): Formicina mutinensis Canestr., Bakar, 12.4., Damin. INV.NO.580 (1 F): Formicina mutinensis Canestr., Glavotok, Damin. INV.NO.581 (2 F): Formicina mutinensis var. pallida Canestr., Bakar, Damin. INV.NO.1201 (1 F, 1 M): Formicina mutinensis Canestr., 22.3. INV.NO.1248 (1 F): Formicina mutinensis Canestr., Omišalj, 7.6. INV.NO.2360 (2 F): Mangora acalypha Walck.,

Diplocephalus alpinus (O. P.-Cambridge, 1872)

INV.NO.1314 (1 F): Diplocephalus connectens Kulcz., INV.NO.1324 (1 F): Diplocephalus connectens Kulcz., Bitoraj (podnožje), 26.6.1892.

Diplocephalus cristatus (Blackwall, 1833)

INV.NO.2397 (1 M): Epeira umbratica Clerck., Topusko, 8.1891.

Diplostyla concolor (Wider, 1834)

INV.NO.1326 (1 F): Bathyphantes concolor Wid., Kecskemet, 1890.

Erigone dentipalpis (Wider, 1834)

INV.NO.809 (2 F, 4 M): Erigone dentipalpis Wid., Bakar (zid). INV.NO.1315 (1 F): Erigone dentipalpis Wid., Starigrad. INV.NO.1317 (1 M): Lephthyphantes flavipes Blackw., INV. NO.2245 (2 M): Uloborus Walckenaeri Latr., Bakar (Srebrač), 07.09., Damin. INV.NO.2300 (1 F): Attus Daminii Chyzer., Baška, Krk, 08.06.1987., Damin.

Floronia bucculenta (Clerck, 1757)

INV.NO.2457 (1 F): Theridium bimaculatum L. Koch, Topusko.

Frontinellina frutetorum (C. L. Koch, 1834)

INV.NO.843 (1 JUV*): Linyphia frutetorum C.L. Koch, Bakar, Damin. INV.NO.843 (15 F, 19M): Linyphia frutetorum C.L. Koch, Bakar, Damin. INV.NO.844 (1 F): Linyphia frutetorum C.L. Koch, Polače, Damin. INV.NO.845 (1 F): Linyphia frutetorum var. punctiventris Chyz. et Kulcz., Buk Krke, Damin. INV.NO.1705 (2 M): Linyphia frutetorum C.L. Koch, Buk Krke. INV.NO.1720 (5 JUV*): Linyphia frutetorum C.L. Koch, Bakar. INV. NO.1725 (1 SUBM*): Linyphia frutetorum C.L. Koch, Bribir-Novi, 15.04.1897. INV.NO.1737 (2 F, 4SUBF* $\left.{ }^{*} 6 \mathrm{SUBM}^{*}, 3 \mathrm{JUV}^{*}\right)$ : Linyphia frutetorum var. punctiventris Chyz. et Kulcz., Buk Krke. INV.NO.2336 (1 F): Lycosa annulata Thor., Polje, Krk, 3.8.1899., Damin. INV. 
NO.2411 (1 F): Linyphia pusilla Sund., Bakar. INV.NO.1298 (1 JUV*): Linyphia frutetorum var. punctiventris Chyz. et Kulcz., Lokrum. INV.NO.1303 (1 JUV*): Linyphia frutetorum var. punctiventris Chyz. et Kulcz., Bribir-Novi, 15.4.1897.

Gonatium hilare (Thorell, 1875)

INV.NO.18 (1 F, 1 M): Gonatium hilare Thor., Omisalj, Damin. INV.NO.1292 (1 M): Gonatium hilare Thor., Split, 27.7.

Gongylidium rufipes (Linnaeus, 1758)

INV.NO.1325 (1 F): Gongylidium rufipes Linn., Osijek, 1898.

Hylyphantes graminicola (Sundevall, 1830)

INV.NO.2246 (1 F): Aelurillus gilvus E. Sim.

Lepthyphantes leprosus (Ohlert, 1865)

INV.NO.265 (2 F): Lephthyphantes leprosus Ohlert., Mrzla vodica, Damin. INV. NO.489 (2 F): Pirata piraticus Clerck., Topusko, 8.1891. INV.NO.1305 (1 F, 2 M, 1 SUBM*): Lephthyphantes leprosus Ohlert., Lokve, 17.8. INV.NO.1327 (1 F): Lephthyphantes leprosus Ohlert., Topusko.

Lepthyphantes sp.

INV.NO.2421 (1 JUV): Pirata piraticus Clerck., 28.07.1915.

Linyphia hortensis Sundevall, 1830

INV.NO.840 (2 F): Linyphia peltata Wid., Zagreb. gora, Damin. INV.NO.1313 (1 F, 1 SUBM$^{*}$ ): Linyphia hortensis Sund., Zagreb, 1897. INV.NO.1318 (1 M): Linyphia hortensis Sund., Sljeme, 6.9. INV.NO.1318 (1 F): Linyphia hortensis Sund., Sljeme, 6.9.

Linyphia sp.

INV.NO.1308 (2SUBM, 2 JUV): Linyphia sp., Skradin, 23.7.1894.

Linyphia triangularis (Clerck, 1757)

INV.NO.847 (13 F, 4M, $\left.4 \mathrm{SUBF}^{*}, 1 \mathrm{SUBM}^{*}, 5 \mathrm{JUV}^{*}\right)$ : Linyphia triangularis Clerck., Bakars. Draga, Damin. INV.NO.848 (2 F, 2 M): Linyphia triangularis Clerck., Osijek, Damin. INV.NO.849 (2 F): Linyphia triangularis Clerck., Fužine, 27.8.1889., Damin. INV. NO.1264 (9 F): Linyphia triangularis Clerck. INV.NO.1265 (5 F, 11 M, 4 SUBF*): Linyphia triangularis Clerck., Jasikovac, 18.7.1902. INV.NO.1266 (2 F): Linyphia triangularis Clerck., Osijek, 1907., Dr. Langhoffer. INV.NO.1267 (3 F): Linyphia triangularis Clerck., Osijek, 12.1898. INV.NO.1272 (1 SUBF*): Linyphia triangularis Clerck. INV.NO.1273 (24 F, 1 M, 2 JUV*$^{*}$ ): Linyphia triangularis Clerck., Topusko, 8.1891. INV.NO.1277 (1 JUV*): Linyphia triangularis Clerck., Zagreb, 1897., Dr. Langhoffer. INV.NO.1278 (1 M): Linyphia triangularis Clerck., Plitvice, 18.8.1898. INV.NO.1282 (2 F): Linyphia triangularis Clerck., Zagreb, 1897. INV.NO.1302 (8JUV*): Linyphia triangularis Clerck., Topusko, 8.1891.

Linyphiidae gen. sp.

INV.NO.2301 (1 JUV): Attus Daminii Chyzer., Baška, Krk, 08.06.1987., Damin. INV. NO.2372 (1 F): Thanatus vulgaris E. Sim., ?Bakar.

Megalepthyphantes nebulosus (Sundevall, 1830)***

INV.NO.264 (1 SUBM*): Lephthyphantes nebulosus Sund., Topusko, Damin. 
Micrargus herbigradus (Blackwall, 1854)*

INV.NO.1207 (1 M): Lophomma herbigradum Black., Lokve (špilja Golubnjak), 18.7.1900.

Neriene emphana (Walckenaer, 1841)

INV.NO.846 (2 F): Linyphia emphana Walck., Crni lug, 4.8.1889., Damin. INV.NO.1287 (2 F): Linyphia emphana Walck., 1902., Broz. INV.NO.1992 (1 F): Linyphia emphana Walck., Zemun.

Neriene furtiva (O. P.-Cambridge, 1871)

INV.NO.1709 (2 F): Linyphia pusilla Sund., Bakar. INV.NO.2049 (1 F): Linyphia pusilla Sund.

Neriene montana (Clerck, 1757)

INV.NO.1296 (1 M): Linyphia montana Clerck. INV.NO.1307 (1 SUBM*): Linyphia montana Clerck. INV.NO.1312 (3 JUV*): Linyphia montana Clerck., Topusko, 8.1891. INV. NO.1319 (6SUBM* 7 JUV*): Linyphia montana Clerck., Osijek. INV.NO.1987 (1 F): Nesticus cellulanus Clerck., Plitvice, 6.8.1902.

Neriene peltata (Wider, 1834)*

INV.NO.839 (3 F): Linyphia peltata Wid., Crni lug, 21.8.1889., Damin.

Neriene radiata (Walckenaer, 1841)

INV.NO.1297 (1 F): Linyphia marginata C.L. Koch, Delnice, 5.7.1891. INV.NO.1323 (1 F): Linyphia marginata C.L. Koch. INV.NO.1328 (1SSP*): Linyphia marginata C.L. Koch, Topusko, 8.1891.

Neriene sp.

INV.NO.841 (2 JUV): Linyphia pusilla Sund., Bakar, 22.3., Damin. INV.NO.2258 (1 JUV): Tetragnatha extensa Linn., Osijek, Damin.

Pelecopsis elongata (Wider, 1834)

INV.NO.1316 (30 F, 10M): Brachycentrum elongatum Wid. INV.NO.1332 (2 F): Brachycentrum elongatum Wid. INV.NO.2374 (1 F): Meta Merianae Scop., Bakars. Draga.

Prinerigone vagans (Audouin, 1826)

INV.NO.1306 (1 F): Erigone speciosa Thor., Kecskemet, 1890. INV.NO.808\#: Erigone vagans Wid., Bakar, Damin. INV.NO.1320 (1SSP*): Erigone vagans Wid., Osijek, 1898.

Silometopus reussi (Thorell, 1871)*

INV.NO.1322 (1 F): Cnephaloctes interjectus O.P. Cambr., Bakar.

Stemonyphantes lineatus (Linnaeus, 1758)****

INV.NO.1259 (1SSP*): Stemonyphantes bucculentus Clerck., Topusko, 08.1891.

Tenuiphantes alacris (Blackwall, 1853)

INV.NO.1321 (1 F): Lephthyphantes terricola?.

Tenuiphantes flavipes (Blackwall, 1854)

INV.NO.2321 (1 F): Pistius truncatus Pallas., Plitvice. 
Tenuiphantes tenebricola (Wider, 1834)

INV.NO.1301 (3 F): Lephthyphantes tenebricola, Vrata. INV.NO.1311 (1 F): Lephthyphantes tenebricola, Bitoraj (podnožje), 6.6.1892.

Trichoncus affinis Kulczyński, 1894

INV.NO.62 (1 F): Trichoncus affinis Kulcz., Bakar, 24.10., Damin. INV.NO.63 (2 F, 1 M): Trichoncus affinis Kulcz., Martinšćica, 05.05.,Damin.

Trichoncus sordidus Simon, 1884

INV.NO.61 (2 F , 2 M): Trichoncus scrofa E. Sim., Crikvenica, 25.02., Damin. INV. NO.1299 (4 F): Trichoncus affinis Kulcz., Bakar (vrtić), 27.04.1890. INV.NO.1304 (1 F, 2 M): Trichoncus sordidus E. Sim., Bakar (pred kupal.), 10.10., Damin. INV.NO.1309 (1 F): Trichoncus sordidus E. Sim., INV.NO.1905 (2 F): Trichoncus sordidus E. Sim., Bakar. INV.NO.2248 (2 F): Trichoncus affinis Kulcz., Bakar, 24.10., Damin. INV.NO.2249 (1 F): Trichoncus affinis Kulcz., Martinšćica, 05.05., Damin. INV.NO.2355 (3 F): Dipoena croatica Chyz., Omišalj, Damin N.

Walckenaeria antica (Wider, 1834)*,**

INV.NO.2438 (1SSP*): Walckenaera antica Wid., Lunte?.

\section{Liocranidae Simon, 1897}

Liocranum perarmatum Kulczyński, 1897***

INV.NO.1409 (1 JUV*): Liocranum perarmatum Kulcz., Kraljevica, 31.7.1899.

Liocranum rupicola (Walckenaer, 1830)

INV.NO.1414 (1 F): Liocranum rupicola Walck., Bakar (kanal), 20.3.

Mesiotelus tenuissimus (L. Koch, 1866)

INV.NO.920 (22 F, 10M, 2SSP.*): Liocranum tenuissimum L. Koch, Gasperini. INV. NO.921 (1 M): Liocranum tenuissimum L. Koch, Split, Damin. INV.NO.921 (3 F): Liocranum tenuissimum L. Koch, Split, Damin. INV.NO.922 (1 M): Liocranum tenuissimum L. Koch, Bakar, Damin. INV.NO.922 (8 F): Liocranum tenuissimum L. Koch, Bakar, Damin. INV. NO.1479 (1 M): Liocranum tenuissimum L. Koch, Bakar, 1.3. INV.NO.1546 (1 M): Liocranum tenuissimum L. Koch, Bakar, 16.9.1890. INV.NO.2048 (1 F): Liocranum tenuissimum L. Koch, Bakar. INV.NO.2393 (1 F): Liocranum rupicola Walck., Bakar (kanal), 20.3. INV. NO.2425 (1 F): Clubiona sp., Bakar.

\section{Lycosidae Sundevall, 1833}

Alopecosa accentuata (Latreille, 1817)

INV.NO.461 (1 F): Tarentula sp. INV.NO.1012 (2 F, 2 M, 1SSP*): Tarentula accentuata Thorell, Osijek. INV.NO.1848 (2 F): Tarentula accentuata Thorell, Osijek. INV.NO.2031 (2 F, $1 \mathrm{M})$ : Tarentula accentuata Thorell. INV.NO.2227 (1 M): Tarentula barbipes, Grabarje, 1912.

Alopecosa albofasciata (Brullé, 1832)

INV.NO.94 (3 F, 5 M, 3 SUBF*, 24 JUV*$^{*}$ ): Tarentula albofasciata Brülle. INV.NO.95 (2 F, $1 \mathrm{M}$ ): Tarentula albofasciata Brulle, Skradin, Damin. INV.NO.1013 (4 F, 11 M, 4 SUBF* 5 SUBM$^{*}, 2$ JUV*): Tarentula albofasciata Brülle. INV.NO.1014 (1 F): Tarentula albofasciata Brülle, Sv. Juraj, 17.07.1899., Damin. INV.NO.1015 (2 F): Tarentula albofasciata Brülle, 
Kotor, Damin. INV.NO.1755 (1 M): Tarentula albofasciata Brülle, Bakar. INV.NO.2228 (1 M): Tarentula cursor Hahn., Jablanac, 10.1932.

\section{Alopecosa cuneata (Clerck, 1757)}

INV.NO.2361 (1 F): Tarentula pulverulenta v. aculeata CI., Risnjak, 1000-150 m. INV. NO.2362 (1 JUV*): Tarentula pulverulenta v. aculeata CI., Risnjak, 1000-150 m. INV. NO.2363 (1 SUBF*, $1 \mathrm{SUBM}^{*}$ ): Tarentula pulverulenta v. aculeata CI., Risnjak, 1000-150 m.

\section{Alopecosa cursor (Hahn, 1831)}

INV.NO.529 (1 JUV*): Tarentula cursor Hahn. INV.NO.572 (1 F, 1 M): Tarentula cursor Hahn. INV.NO.2168 (1 F): Tarentula sp., Caska, 14.06.1958. INV.NO.2255 (1 F): Tarentula albofasciata Brülle.

Alopecosa inquilina (Clerck, 1757)

INV.NO.540 (1 F): Tarentula inquiliana Clerck., Nad Senjom (Velebit). INV.NO.573 (1 M): Tarentula inquiliana Clerck. INV.NO.574 (2 F, 1 M): Tarentula inquiliana Clerck., Split, Damin. INV.NO.2034 (1 M): Tarentula inquilina Clerck., INV.NO.2317 (1 F): Tarentula cursor Hahn.

Alopecosa mariae (Dahl, 1908)

INV.NO.680 (1 F, 1 M): Tarentula striatipes Dolesch, Osijek.

Alopecosa pulverulenta (Clerck, 1757)

INV.NO.510 (1 F): Tarentula pulverulenta v. aculeata, Bosna, Apfelback. INV.NO.683 (1 F, 3 M): Tarentula pulverulenta, Osijek. INV.NO.1753 (1 M): Tarentula pulverulenta, Podsused, 25.09.1896.

Alopecosa schmidti (Hahn, 1835)

INV.NO.1698 (1 F, 2 M): Tarentula Eichwaldii Thor., Krakow.

Alopecosa solitaria (Herman, 1879)

INV.NO.826 (1 F, 1 M, 1 JUV*): Tarentula solitaria O. Herm. INV.NO.827 (4 SUBF*): Tarentula solitaria O. Herm., Ogulin, Damin. INV.NO.828 (1 M, 1 SUBF*): Tarentula solitaria O. Herm., Bakar (Turčina), Damin. INV.NO.1763 (1 F): Tarentula solitaria O. Herm., Jablanac. INV.NO.2123 (2 M): Tarentula sp., Kolansko blato, 04.10.1957. INV. NO.2420 (1SSP*): Tarentula solitaria O. Herm., Jablanac.

\section{Alopecosa sp.}

INV.NO.352 (1 SUBM): Lycosa morosa L. Koch, Kostrena, Sv. Barb., 2.5.1890. INV. NO.461 (1 SUBF): Tarentula sp. INV.NO.518 (2 JUV): Tarentula sp. INV.NO.543 (1 SUBM): Tarentula leopardus, Osijek, 1897., Langhoffer. INV.NO.575 (1 JUV*): Tarentula lacustris Sim., INV.NO.576 (1 JUV): Tarentula lacustris Sim., Split. INV.NO.1758 (1 SUBF): Pirata piraticus Clerck., Plitvice, 26.08.1902. INV.NO.2101 (1 F): Tarentula sp., Gaska, o. Pag, 12.06.1958. INV.NO.2125 (1 F): Tarentula sp., St. Novalja, o. Pag, 24.06.1960. INV.NO.2331 (1 SUBF, $1 \mathrm{JUV}^{*}$ ): Lycosa morosa L. Koch, Glavotok (Krk), Damin.

Alopecosa sulzeri (Pavesi, 1873)***

INV.NO.564 (1 SUBF*): Trochosa Sulzeri Pav. INV.NO.1786 (2 F, 1 SUBF*, 1SSP*): Trochosa Sulzeri Pav., Bakar. 
Alopecosa taeniata (C. L. Koch, 1835)*

INV.NO.499 (1 M): Tarentula pulverulenta v. aculeata CI., Snj., Risnjak, Lazac - 1069 m, 18.06., Varićak. INV.NO.824 (2 F): Tarentula pulverulenta v. aculeata CI., Risnjak, 1000-150 m. INV.NO.825 (1 F): Tarentula pulverulenta v. Gastainensis.

Alopecosa trabalis (Clerck, 1757)

INV.NO.681 (1 F, 1 M): Tarentula trabalis Clerck., Ogulin. INV.NO.681 (): Tarentula trabalis Clerck., Ogulin. INV.NO.682 (1 F, 1 M, 1 SUBM*): Tarentula trabalis Clerck. INV. NO.2057 (1 SUBM*): Tarentula trabalis Clerck.

Arctosa cf. maculata (Hahn, 1822)

INV.NO.2242 (1 F): Arctosa sp., Lokve, 07.1930.

Arctosa cinerea (Fabricius, 1777)

INV.NO.223 (1 SUBM*): Trochosa cinerea Fabr. INV.NO.224 (2 F, 2 M): Trochosa cinerea Fabr., Dolina. INV.NO.2365 (1 F): Tarentula pulverulenta v. Gastainensis.

Arctosa leopardus (Sundevall, 1833)

INV.NO.258 (1 M $\left.{ }^{*}\right)$ : Trochosa leopardus Sund. INV.NO.575 (3 F, 1 M): Tarentula lacustris Sim. INV.NO.576 (1 F): Tarentula lacustris Sim., Split. INV.NO.568 (1 JUV*): Trochosa leopardus Sund., Osijek, 1899. INV.NO.258 (2SUBM*): Trochosa leopardus Sund.

Arctosa maculata (Hahn, 1822)

INV.NO.722 (2 F, 2 M): Trochosa amylacea C.L. Koch, Risnjak, Damin. INV.NO.723 (4 F, $1 \mathrm{M})$ : Trochosa amylacea C.L. Koch, Mrzla vodica, Damin. INV.NO.2238 (1 F): Arctosa sp., Lokve (Bukovac), 07.1929.

Arctosa perita (Latreille, 1799)

INV.NO.78 (1 M, 1 SUBM*): Trochosa perita Latr., Split, Damin.

Arctosa sp.

INV.NO.293 (2 SUBM, 1 JUV): Drassodes minusculus (= Dalmat L. Koch, Gasperini. INV.NO.512 (1 JUV): Tarentula sp. INV.NO.731 (4 SUBF, 1 SUBM, 1 JUV): Lycosa morosa L. Koch, Glavotok.

Arctosa variana C. L. Koch, 1847

INV.NO.220 (2 F, 2 M): Trochosa variana C.L. Koch. INV.NO.221 (1 F, 1 M): Trochosa variana C.L. Koch, Šibenik, Damin. INV.NO.222 (1 F, 3 M): Trochosa variana C.L. Koch, Bakar, Damin. INV.NO.1773 (1 M): Trochosa variana C.L. Koch, Bakar, 05.05. INV.NO.222 $\left(3 \mathrm{SUBF}^{*}\right)$ : Trochosa variana C.L. Koch, Bakar, Damin.

Aulonia albimana (Walckenaer, 1805)

INV.NO.1 (7 F, 1 M, 12 SUBM*, 6 JUV*): Aulonia albimana, Bakarac, Damin. INV.NO.248 (1 SUBM*): Aulonia albimana, Grižane, 14.4.1900. INV.NO.266 (1 F): Aulonia albimana, Žakalj.

Geolycosa vultuosa (C. L. Koch, 1838)

INV.NO.410 (1 F): Trochosa infernalis Motsch., Split, Damin. INV.NO.411 (2 F, 2 JUV*): Trochosa infernalis Motsch., Zemun, 1899. INV.NO.412 (4 F, 1 SUBF*): Trochosa infernalis Motsch., Zemun, 1899. INV.NO.413 (2 F): Trochosa infernalis Motsch. INV.NO.517\#: Trochosa infernalis Motsch., Split. INV.NO.1766 (1 F): Trochosa infernalis Motsch., Zemun, 
1899., Canjuga. INV.NO.1766 (3 JUV*): Trochosa infernalis Motsch., Zemun, 1899., Canjuga. INV.NO.1770 (1 SUBF*): Trochosa infernalis Motsch., Osijek. INV.NO.1770 (1 F): Trochosa infernalis Motsch., Osijek.

Hogna radiata (Latreille, 1817)

INV.NO.347 (4 SUBF*, 2 JUV*): Tarentula radiata Latr. INV.NO.542 (1 JUV*): Tarentula radiata Latr., Bribir-Novi, 15.4.1897. INV.NO.565 (1 JUV*): Tarentula radiata Latr., Podsused, 25.5.1896. INV.NO.829 (20JUV*): Tarentula radiata Latr., Bakar, 30.5., Damin. INV.NO.830 (1 M, 2 JUV*): Tarentula radiata Latr., Šibenik, Damin. INV.NO.831 (1 SUBF*, $1 \mathrm{SUBM}^{*}$ ): Tarentula radiata Latr., Jablanac, 11.7., Damin. INV.NO.1016 (3 M, 1 JUV*): Tarentula radiata Latr., Povile, Damin. INV.NO.1017 (2 F, 1 M, 1 SUBF* 1 SUBM ${ }^{*}, 143$ JUV*): Tarentula radiata Latr., Bakar, 7.10.1891., Damin. INV.NO.1019 (4 SUBF*, $1 \mathrm{M}$ ): Tarentula radiata Latr., Senj, 6.7.1899., Damin. INV.NO.1020 (1 F, 1 SUBF*, 1 SUBM*, 24 $\left.\mathrm{JUV}^{*}\right)$ : Tarentula radiata Latr. INV.NO.1774 (1 JUV*): Tarentula radiata Latr., Buk Krke. INV.NO.1788 (1 F): Tarentula sp., otok Sv. Marko, 16.08.1902. INV.NO.1794 (1 F): Tarentula radiata Latr., Draga, 16.07.1908., K. Babić. INV.NO.1840 (1 F): Pirata knorrii Scop., Zagreb, Sofijin put, 08.07.1905. INV.NO.1843 (1 F): Tarentula radiata Latr., Valpovo (zvjerinjak), 05.08.1905. INV.NO.1845 (1 F): Tarentula radiata Latr., Draga, 09.08.1908., K. Babić. INV. NO.2118 (3 F): Tarentula sp., Kolansko blato, 04.10.1957. INV.NO.2154 (1 F): Tarentula sp., Kolansko blato, 04.10.1957. INV.NO.2161 (1 F): Tarentula sp., St. Novalja, o. Pag, 04.10.1957. INV.NO.2162 (1 F): Tarentula sp., Kolansko blato, o. Pag, 04.10.1957. INV.NO.2172 (1 F): Trochosa sp., o. Susak, 01.10.1957. INV.NO.2203 (2 F, 2 M): Tarentula sp. INV.NO.2226 (1 F): Tarentula sp., Vermač (Kotor), 19.07.1931.

\section{Hogna sp.}

INV.NO.2091 (1 SUBF): Trochosa sp., o. Pag, Kolansko blato, 27.06.1964. INV.NO.2107 (5SUBF): Trochosa sp., Caska, 14.06.1958. INV.NO.2115 (1 SUBM): Tarentula sp., Caska, 24.06.1960. INV.NO.2117 (1 SUBF): Trochosa sp. Caska, 24.06.1960. INV.NO.2142 (4 SUBF, 1 JUV): Tarentula sp., o. Pag, Velo blato, 04.07.1956. INV.NO.2177 (1 JUV): Trochosa sp., Caska, 09.06.1958. INV.NO.2189 (4 JUV): Trochosa sp., Kolansko blato, 11.06.1958. INV. NO.2192 (4SUBF): Tarentula sp., Velo blato, o. Pag, 04.07.1956. INV.NO.2195 (1 SUBF): Trochosa sp., Stara novalja, o. Pag, 13.06.1958. INV.NO.2196 (2 JUV): Trochosa sp., Caska, o. Pag, 10.07.1958. INV.NO.2352 (1 JUV): Anyphaena accentuata Walck., Bakar, 12.04.1892., Damin. INV.NO.2356 (1 JUV): Teutana triangulosa Walck., Bakar, Primorje, Damin N. INV. NO.2424 (1 JUV): Clubiona sp., Bakar.

Lycosa singoriensis (Laxmann, 1770)

INV.NO.2087 (1 F): Hogna singorensis (Laxm.), Novi Vrbas, 25.03.1933. INV.NO.2231 (15 F): Hogna singorensis (Laxm.), Stari Vrbas, 18.04.1933.

\section{Lycosa tarantula (Linnaeus, 1758)}

INV.NO.77 (1 M): Trochosa ruricola Deg. INV.NO.569 (1 F): Tarentula Apuliae Walck., Vrbica (Dalm.), 22.07.1894., Damin. INV.NO.1781 (1 SUBF*, $\left.1 \mathrm{JUV}^{*}\right)$ : Tarentula Apuliae Walck. INV.NO.1789 (1 F): Tarentula Apuliae Walck., Vrbica (Vrana), 22.07.1894., Damin. INV.NO.1844 (2 JUV*): Tarentula Apuliae Walck., Muć. INV.NO.1844 (6SUBF*): Tarentula Apuliae Walck., Muć. INV.NO.2076 (1 M): Hogna tarentula, o. Unije, 27.06.1963. INV. NO.2079 (1 SUBF*): Hogna tarentula, Povljana, o. Pag, 01.07.1960. INV.NO.2169 (1 M): Hogna tarentula, Kolansko blato, o. Pag, 01.07.1955. INV.NO.2173 (1 F): Hogna tarentula, Kolani, o. Pag/ Novalja-Kolansko blato, o. Pag, 26.7.1956./ 1.7.1955. INV.NO.539\#: Tarentula Apuliae Walck., Vrbica (Vrana). INV.NO.541 (1SSP*): Tarentula Apuliae Walck., Vrbica 
(Vrana). INV.NO.570 (1 SUBF*): Tarentula Apuliae Walck., Nad Bukom Krke, Damin. INV. NO.571 (1 SUBF*, 1 SUBM*): Tarentula Apuliae Walck., Damin. INV.NO.710 (2 JUV*, 1 $\left.\mathrm{SUBF}^{*}\right)$ : Lycosa tarentula.

Pardosa agrestis (Westring, 1861)

INV.NO.170 (1 M): Lycosa monticola Clerck., Osijek, 1897., Dr. Langhoffer. INV.NO.183 (1 F): Lycosa agrestis Westr., 1902. INV.NO.197 (1 SUBF*): Lycosa agrestis Westr., Osijek, 7.1901., Rössler. INV.NO.705 (3 F): Lycosa agrestis Westr., Bakar, 8.8., Damin. INV.NO.706 (3 F, 2 M): Lycosa agrestis Westr., Vrana (Dalm.), Damin.

Pardosa agricola (Thorell, 1856)

INV.NO.719 (2 F): Lycosa agricola Thorell, Ogulin, Damin.

Pardosa alacris (C. L. Koch, 1833)

INV.NO.2307 (1 M): Tarentula pulverulenta v. aculeata CI.Snj., Risnjak, Lazac - 1069 m, 18.06., Varićak. INV.NO.2308 (2 F): Tarentula pulverulenta v. aculeata CI., Snj., Risnjak, Lazac - 1069 m, 18.06., Varićak. INV.NO.2328 (3 M): Lycosa lugubris Walck., Ogulin. INV. NO.2329 (1 M): Lycosa lugubris Walck., Ogulin.

Pardosa amentata (Clerck, 1757)

INV.NO.162 (1 F): Lycosa amentata CI., Zagreb, 9.7.1902., E. Peičić. INV.NO.267 (1 F): Lycosa amentata CI., Fužine, 6.6.1897. INV.NO.303 (1 M): Lycosa amentata CL. Koch, Ogulin, 4.6.1898. INV.NO.326 (1 F, 1 M): Lycosa amentata Cl., Križevci, 11.9.1897. INV. NO.715 (5M): Lycosa amentata CI., Bakar, Damin. INV.NO.715 (5 F): Lycosa amentata CI., Bakar, Damin. INV.NO.1751 (1 F): Lycosa monticola Clerck., Babina greda, 17.07.1902. INV.NO.1756 (1 F): Lycosa monticola Clerck., Babina greda, 17.07.1902. INV.NO.1769 (1 F): Lycosa monticola Clerck., Mitrovica, 25.04.1911. INV.NO.2335 (1 F): Lycosa annulata Thor., Rijeka, Bakar, Crikvenica, Damin.

Pardosa cf. albatula (Roewer, 1951)

INV.NO.2029 (1 F): Lycosa albata L. Koch.

Pardosa cf. blanda (C. L. Koch, 1833)*

INV.NO.709 (2 F): Lycosa cursoria C.L. Koch, Vratnik, Damin.

Pardosa hortensis (Thorell, 1872)

INV.NO.164 (1 F): Lycosa annulata Thor., Plitvice, 27.8.1902. INV.NO.167 (2 JUV*): Lycosa annulata Thor., Topusko, 8.1891. INV.NO.184 (1 F): Lycosa annulata Thor. INV. NO.207 (2 F, 3 M): Lycosa annulata Thor., Bribir-Novi, 15.09.1892. INV.NO.251 (1 F, 2 M): Lycosa annulata Thor., Grižane, 14.4. INV.NO.253 (2 F): Lycosa annulata Thor., Podsused, 5.5.1896. INV.NO.268 (4 F, 3 M): Lycosa annulata Thor. INV.NO.327 (1 M): Lycosa annulata Thor., lzvor Rječine, 23.5. INV.NO.331 (1 M): Lycosa annulata Thor., Bakar. INV.NO.717 (15 F, 4M): Lycosa annulata Thor., Rijeka, Bakar, Crikvenica, Damin. INV.NO.720 (1 F): Lycosa annulata Thor., Polje, Krk, 3.8.1899., Damin. INV.NO.721 (1 M, 1 JUV*): Lycosa annulata Thor., Split, Damin. INV.NO.1018 (1 F, 1 SUBM* $\left.{ }^{*} 1 \mathrm{JUV}^{*}\right)$ : Tarentula hortensis. INV.NO.1691 (2 F, 2 M): Lycosa annulata Thor., Grižane, 14.4.1897. INV.NO.1761 (1 F): Lycosa annulata Thor., Osijek, 04.1899. INV.NO.1767 (1 F): Lycosa monticola Clerck., Fruška gora, Vijenac, 28.05.1910. INV.NO.2333 (4 SUBF*, 10SUBM $\left.{ }^{*}, 8 \mathrm{JUV}^{*}\right)$ : Lycosa annulata Thor., Rijeka, Bakar, Crikvenica, Damin. 
Pardosa lugubris (Walckenaer, 1802)

INV.NO.254 (4 JUV*): Lycosa lugubris Walck., Topusko, 8.1891. INV.NO.304 (2 F, 1 JUV*): Lycosa lugubris Walck., Bag, 1902., Broz. INV.NO.707 (3 F): Lycosa lugubris Walck., Ogulin. INV.NO.708 (10 F, 2 M, 1 JUV*): Lycosa lugubris Walck., Ogulin. INV.NO.1752 (1 F): Lycosa palustris Linn., Zagreb-Cmrok, 04.1906. INV.NO.1759 (1 F): Lycosa monticola Clerck., Sošice, 18.06.1917.

Pardosa monticola (Clerck, 1757)

INV.NO.187 (1 F): Lycosa monticola Clerck., Bitoraj-podnožje, 26.6. INV.NO.245 (1 F): Lycosa monticola Clerck., Delnice, Kernic. INV.NO.1750 (1 JUV*): Lycosa monticola Clerck., Zagreb, 08.1904. INV.NO.1756 (3 JUV*): Lycosa monticola Clerck., Babina greda, 17.07.1902.

Pardosa morosa (L. Koch, 1870)

INV.NO.712 (1 JUV*): Lycosa morosa L. Koch, Sv. Juraj, Damin. INV.NO.713 (1 F): Lycosa morosa L. Koch, Glavotok (Krk), Damin. INV.NO.726 (6M): Lycosa morosa L. Koch, Bakar, Damin. INV.NO.1213 (1 F, 1 M): Amaurobius sp., Kostrena Sv. Barbara, 02.05.1890.

Pardosa nebulosa (Thorell, , 1872)

INV.NO.561 (4SUBF*, 7SUBM*): Lycosa nebulosa Thor., Kecskemet, 03.1890., Salzteich. INV.NO.730 (3 F): Lycosa nebulosa Thor.,

Pardosa nigra (C. L. Koch, 1834)

INV.NO.2061 (1 M): Lycosa nigra C.L. Koch.

Pardosa paludicola (Clerck, 1757)

INV.NO.716 (4 F): Lycosa paludicola Clerck., Osijek.

Pardosa palustris (Linnaeus, 1758)

INV.NO.2062 (3 F, 1 M): Lycosa palustris Linn.

Pardosa prativaga (L. Koch, 1870)

INV.NO.464 (1 F): Lycosa prativaga L. Koch, 1901., Trgovčević. INV.NO.1784 (1 M): Lycosa amentata CI., Boljevci (zidine), 21.05.1912.

Pardosa proxima (C. L. Koch, 1847)

INV.NO.501 (1 F): Lycosa proxima C.L. Koch, Grižane, 14.4. INV.NO.511 (1 F): Lycosa proxima C.L. Koch, Bribir-Novi, 15.4.1897. INV.NO.530 (1 M): Lycosa proxima C.L. Koch, 22.7. INV.NO.544 (1 M): Lycosa proxima C.L. Koch, Osijek, 4.1899. INV.NO.549 (1 SUBF*): Lycosa proxima C.L. Koch, Kecskemet, 03.1890., Salzteich. INV.NO.718 (2 F): Lycosa proxima C.L. Koch. INV.NO.727 (3 SUBM*, $4 \mathrm{JUV}^{*}$ ): Lycosa proxima C.L. Koch, Zemun, Damin. INV.NO.728 (1 F, 1 SUBM*): Lycosa proxima C.L. Koch, Bakar, Damin. INV.NO.729 (1 F): Lycosa proxima C.L. Koch, Vrana, Damin. INV.NO.1678 (1 F): Lycosa proxima C.L. Koch, Vrbica.INV.NO.2332 (1 F): Lycosa annulata Thor., Rijeka, Bakar, Crikvenica, Damin.

\section{Pardosa sp.}

INV.NO.562 (1 SUBM, 1 JUV): Lycosa sp., na pijesku kod Đurdevca. INV.NO.726 (2 JUV): Lycosa morosa L. Koch, Bakar, Damin. INV.NO.2395 (1 JUV): Chiracanthium Letochae, Kraljičin zdenac, 14.06.1897., Dr. Langhoffer. 
Pardosa vittata (Keyserling, 1863)

INV.NO.711 (1 F): Lycosa vittata Keys. Crikvenica, Damin. INV.NO.2124 (1 F): Lycosa vittata Keys., Caska, 09.06.1958. INV.NO.2128 (2 M): Lycosa sp., Caska, 25.06.1960.

Pardosa wagleri (Hahn, 1822)

INV.NO.714 (1 F, 1 M): Lycosa Wagleri Hahn., Šibenik, Damin.

Pirata piraticus (Clerck, 1757)

INV.NO.504 (1 SUBM*): Pirata piraticus Clerck., Osijek, 7.6.1901. INV.NO.1122 (2 F): Pirata piraticus Clerck., Gasperini. INV.NO.1123 (1SSP*, 1 F, 2 M, 1 SUBM* ${ }^{*} 1$ JUV $^{*}$ ): Pirata piraticus Clerck., Damin. INV.NO.1124 (1 SUBM*, 1 M): Pirata piraticus Clerck., Zemun, Damin. INV.NO.1125 (1 SUBM ${ }^{*}$ ): Pirata piraticus Clerck., Bakar, Damin. INV.NO.1764 (1 F): Dolomedes fimbriatus Clerck., Obeda, 27.05.1912. INV.NO.1787 (1 JUV*): Pirata piraticus Clerck., 17.03.

Pirata piscatorius (Clerck, 1757)

INV.NO.520 (1SSP*): Pirata piscatorius Clerck., Zemun. INV.NO.2043 (3 F, 1 M): Pirata piscatorius Clerck.

Pirata sp.

INV.NO.1768 (1 SUBF): Dolomedes Ilimbatus Hahn., Obeda, 24.05.1912.

Piratula hygrophila (Thorell, 1872)

INV.NO.1760 (2 F): Pirata hygrophilus Thor., Fužine.

Piratula knorri (Scopoli, 1763)

INV.NO.1121 (4 F, 1 M, 2SUBM*, 3 JUV*): Pirata knorrii Scop., Mrzla vodica.

Piratula latitans (Blackwall, 1841)

INV.NO.1126 (2 F, 1 SUBM*, 2 JUV*): Pirata latitans Blackw., Martinšćica, Damin. INV. NO.2183 (1 F): Pirata latitans Blackw., Caska, o. Pag, 14.06.1958.

Trabea paradoxa Simon, 1876

INV.NO.965 (1 F): Philodromus aureolus Clerck verus, Gasperini.

Trochosa hispanica Simon, 1870

INV.NO.75 (1 F): Trochosa ruricola Deg., Senj, Damin. INV.NO.76 (1 F): Trochosa ruricola Deg., Zemun, Damin. INV.NO.345 (2 F): Trochosa ruricola var. Rustica Thor., Izvor Rječine, 22.5. INV.NO.414 (2 F): Trochosa terricola Thor., Zemun. INV.NO.1754 (1 F): Tarentula sp., Gasperini. INV.NO.1762 (1 F): Pirata piraticus Clerck., Bakar. INV. NO.1765 (1 F): Pirata hygrophilus Thor., Valpovo, 01.05.1906. INV.NO.2131 (1 F): Trochosa sp., Caska, 09.06.1958. INV.NO.2330 (2 M): Lycosa tarentula. INV.NO.2337 (1 F): Trochosa ruricola Deg., Szombor.

Trochosa robusta (Simon, 1876)

INV.NO.74 (5 F, 1 M): Trochosa robusta E. Simon. INV.NO.351 (1 M): Trochosa robusta E. Sim., Kecskemet, 3.1890., Salzteich. INV.NO.1861 (1 F): Trochosa sp., Šestine, 17.07.1905.

Trochosa ruricola (De Geer, 1778)

INV.NO.76 (1 JUV*): Trochosa ruricola Deg., Zemun, Damin. INV.NO.415 (2 M): Trochosa terricola Thor. INV.NO.451 (1 F, 1 M): Trochosa ruricola Deg., Kecskemet, 
3.3.1890., Salzteich. INV.NO.469 (1 SUBF*): Trochosa ruricola Deg., Topusko, 8.1891. INV. NO.724 (7 F, 2 M): Trochosa ruricola Deg., Szombor. INV.NO.724 (): Trochosa ruricola Deg., Szombor. INV.NO.1780 (1 F): Pirata piraticus Clerck., Boljevci, 20.05.1912., Langhoffer. INV.NO.2239 (1 F): Trochosa terricola Thor., Lokve (Bukovec), 07.1929. INV.NO.2292 (1 F): Trochosa robusta E. Sim., Kecskemet, 3.1890., Salzteich.

Trochosa sp.

INV.NO.531 (6JUV): Tarentula sp., Zemun. INV.NO.1782 (1 SUBM): Pirata piraticus Clerck., 28.07.1915. INV.NO.2097 (4 SUBF): Trochosa sp., Caska, o. Pag, 12.06.1958.

Trochosa terricola Thorell, $1856^{* * *}$

INV.NO.366 (1 F): Trochosa terricola Thor., Levi draga, 29.07.1902. INV.NO.378 (1 F): Trochosa terricola Thor., Bosna, Apfelbeck. INV.NO.416 (4 F): Trochosa terricola Thor. INV.NO.467 (1 M): Trochosa terricola Thor., Delnice, Kernic. INV.NO.2239 (2 F): Trochosa terricola Thor., Lokve (Bukovec), 07.1929.

Xerolycosa miniata (C. L. Koch, 1834)

INV.NO.677 (1 F): Tarentula miniata C.L. Koch, Krk, Rudine, 6.8.1891., Damin. INV. NO.2060\#: Tarentula miniata, 15.8.1897.

Xerolycosa nemoralis (Westring, 1861)

INV.NO.348 (1 F, 1 M): Tarentula nemoralis Westr., Snježnik; Risnjak; Lazac; 1069 m, 18.06., Varićak. INV.NO.460 (1 F): Tarentula nemoralis Westr., Topusko. INV.NO.679 (3 F, 1 SUBM $^{*}, 1$ JUV$^{*}$ ): Tarentula nemoralis Westr., Mrzla vodica, Damin. INV.NO.1695 (2 F): Tarentula nemoralis Westr., Mrzla vodica. INV.NO.1695 (3 M): Tarentula nemoralis Westr., Mrzla vodica. INV.NO.678 (1 SUBM* 1 JUV*): Tarentula nemoralis Westr., Fužine, 22.8.1889., Damin.

\section{Mimetidae Simon, 1881}

Ero aphana (Walckenaer, 1802)

INV.NO.939 (1 F, 2 M, 1 JUV*): Ero aphana Walck., Rječina, 3.7., Damin. INV.NO.940 (1 F, 1 M, 3 SUBM $^{*}$ ): Ero aphana Walck., Glavotok, Damin. INV.NO.2346 (1 F): Mimetus laevigatus Keys., Bakar, 04.07., Damin.

Erosp.

INV.NO.2346 (2 JUV): Mimetus laevigatus Keys., Bakar, 04.07., Damin.

Ero tuberculata (De Geer, 1778)**

INV.NO.938 (1SSP*): Ero tuberculata Deg., Bakar.

Mimetus laevigatus (Keyserling, 1863)

INV.NO.756 (1 M): Mimetus laevigatus Keys., Gasperini. INV.NO.757 (1 JUV*): Mimetus laevigatus Keys. Porto Palazzo. INV.NO.758 (3 M, 2SUBM ${ }^{*}$, 8JUV*): Mimetus laevigatus Keys. Bakar, 04.07., Damin. INV.NO.759 (1 F, 3 M, 1 SUBM*, 2 JUV*): Mimetus laevigatus Keys. Zemun, 1899. INV.NO.1983 (3 JUV*): Mimetus laevigatus Keys., Bakar. INV.NO.1984 (1 M): Mimetus laevigatus Keys., Bakar, 03.06. INV.NO.1988 (1 F): Mimetus laevigatus Keys., Omišalj. 


\section{Nemesiidae Simon, 1889}

Brachythele media Kulczyński, 1897

INV.NO.28 (1 F, 2 M): Nemesia radiata Kulcz., Bakar, 23.10.1889., Damin. INV.NO.2418 (1 F): Nemesia radiata Kulcz., Bakar.

Brachythele sp.

INV.NO.28 (1 JUV*): Nemesia radiata Kulcz., Bakar, 23.10.1889., Damin. INV.NO.2418 $\left(2 \mathrm{JUV}^{*}\right)$ : Nemesia radiata Kulcz., Bakar.

Nemesia caementaria (Latreille, 1799)\#

INV.NO.29\#: Nemesia caementaria.

Nemesia pannonica Herman, 1879

INV.NO.27 (1 F, 1 M): Nemesia Sauvagesii Dorth., Gasperini. INV.NO.1742 (1 M): Nemesia radiata Kulcz., Bakar. INV.NO.2218 (1SSP*): Nemesia radiata Kulcz., Bombay, di Weinberger.

Gen. sp. indet.

INV.NO.1346 (1SSP*): Coelotes laniger, ?Bakar (kanal), 17.04.

\section{Oecobiidae Blackwall, 1862}

Oecobius sp.

INV.NO.2347 (1 JUV): Runcinia lateralis C.L. Koch, Bakar, Damin.

Uroctea durandi (Latreille, 1809)

INV.NO.102 (9M): Uroctea Durandii Latr., Bakar, Damin. INV.NO.103 (1 JUV*): Uroctea Durandii Latr., Bakar, 28.08.1889., Damin. INV.NO.103 (2 F, 2 M): Uroctea Durandii Latr., Bakar, 28.08.1889., Damin. INV.NO.104 (1 M, 3 JUV*): Uroctea Durandii Latr., Gasperini. INV.NO.1167 (11 JUV*): Uroctea Durandii Latr. INV.NO.1168 (1 JUV*): Uroctea Durandii Latr., Sv. Kuzma, 20.8.1902., Polić. INV.NO.1169 (1 JUV*): Uroctea Durandii Latr., Kraljevica, 21.7.1899. INV.NO.1188 (1 F): Uroctea Durandii Latr., Stari grad, 29.7. INV.NO.1193 (2 $J^{*}{ }^{*}$ ): Uroctea Durandii Latr., Lukovo, 9.7.1899. INV.NO.1197 (2 JUV*): Uroctea Durandii Latr., Malinska, 27.3. INV.NO.1939 (1 JUV*): Uroctea Durandii Latr., Novi, 05.07.1894. INV. NO.1946 (1 M): Uroctea Durandii Latr., Dolac, 06.09.1905. INV.NO.1947 (1 JUV*): Uroctea Durandii Latr., Nad Ponikvama. INV.NO.2112 (1 JUV*): Uroctea Durandii Latr., Novalja, 04.10.1957. INV.NO.2145 (1 M): Uroctea Durandii Latr., Unije, 1-10.09.1963., Magerle. INV. NO.2164 (1 F): Uroctea Durandii Latr., St. Novalja, 04.10.1957.

\section{Oxyopidae Thorell, 1870}

Oxyopes heterophthalmus (Latreille, 1804)

INV.NO.2135 (4 F, 1 M): Oxiopes sp., Stara novalja, o. Pag, 13.06.1958. INV.NO.528 (1 $\left.\mathrm{JUV}^{*}\right)$ : Oxiopes heterophthalamus Latr.

Oxyopes lineatus Latreille, 1806

INV.NO.301 (1 SUBM*): Oxiopes lineatus Latr., Omišalj. INV.NO.523 (2 F): Oxiopes lineatus Latr., Kotor, Muo, Damin. INV.NO.524 (2 F): Oxiopes lineatus Latr., Jablanac, 12.7.1899., Damin. INV.NO.526 (2 F, 2 M): Oxiopes lineatus Latr., Košljun-Krk, Damin. INV. 
NO.1951 (1 F): Oxiopes lineatus Latr., Bakar, 17.07.1903. INV.NO.523 (4 SUBF*): Oxiopes lineatus Latr., Kotor, Muo, Damin. INV.NO.523 (8JUV*): Oxiopes lineatus Latr., Kotor, Muo, Damin. INV.NO.525 (1 JUV*): Oxiopes lineatus Latr., Novi, Damin. INV.NO.525 (1 $\left.\mathrm{SUBM}^{*}\right)$ : Oxiopes lineatus Latr., Novi, Damin.

Oxyopes nigripalpis Kulczyński, 1891

INV.NO.2386 (5 F): Phlegra lineata C. Koch, Gasperini.

Oxyopes ramosus (Martini \& Goeze, 1778)

INV.NO.521 (1 F): Oxiopes ramosus Panz., Povile, 9.8.1899., Damin. INV.NO.522 (1 JUV*): Oxiopes ramosus Panz., Zagreb, Damin. INV.NO.522 (2 F): Oxiopes ramosus Panz., Zagreb, Damin.

Oxyopes sp.

INV.NO.2386 (1 JUV): Phlegra lineata C. Koch, Gasperini.

\section{Philodromidae Thorell, 1870}

Philodromus aureolus (Clerck, 1757)

INV.NO.968 (8JUV*): Philodromus aureolus Clerck verus, Cavtat, Damin. INV.NO.1044 $\left(1 \mathrm{JUV}^{*}\right)$ : Philodromus aureolus Clerck verus, Osijek, 1897., Langhoffer. INV.NO.1050 $\left(6 \mathrm{JUV}^{*}\right)$ : Philodromus aureolus Clerck verus. INV.NO.1089 (1 F): Philodromus aureolus Clerck verus.

Philodromus cespitum (Walckenaer, 1802)

INV.NO.964 (4M): Philodromus aureolus Clerck verus, Gasperini. INV.NO.967 (2 M): Philodromus aureolus Clerck verus, Prevez, Raša, Istra, Damin. INV.NO.969 (2 F): Philodromus aureolus Clerck verus, Bakar, Damin. INV.NO.970 (1 F, 2 M): Philodromus aureolus caespiticola Clerck verus, Ogulin, Damin. INV.NO.974 (2 F, 1 M): Philodromus aureolus pallens Clerck verus, Zemun, 1899., Damin. INV.NO.1084 (1 F): Philodromus aureolus rufolimbatus Clerck verus, Lukovo, 09.07.1899. INV.NO.1105 (1 F): Philodromus aureolus caespiticola Clerck verus, Križevci, 04.09.1897. INV.NO.1952 (1 F): Philodromus margaritatus Clerck., Babina greda, 17.07.1912.

Philodromus collinus C. L. Koch, 1835

INV.NO.863 (2 M, 2 JUV*): Philodromus collinus C.L. Koch, Bitoraj. INV.NO.971 (1 F): Philodromus aureolus marmorata Clerck verus, Bribir, Damin.

\section{Philodromus dispar Walckenaer, 1826}

INV.NO.854 (4M, 1 SUBM*): Philodromus dispar Walck., Bakar (Kopriva), 21.05.1890., Damin. INV.NO.855 (1 F): Philodromus dispar Walck., Kotor, Damin. INV.NO.855 (9JUV*): Philodromus dispar Walck., Kotor, Damin. INV.NO.856 (1 M, $\left.1 \mathrm{JUV}^{*}\right)$ : Philodromus dispar Walck., Rijeka, Damin. INV.NO.1095 (1 JUV*): Philodromus dispar Walck., Žakalj, 17.09. INV.NO.1100 (4 F, 3 JUV*): Philodromus dispar Walck., Bakar. INV.NO.1104 (3 SUBM*): Philodromus dispar Walck. INV.NO.1106 (1 F, 1 M): Philodromus dispar Walck., Izvor Rječine, 25.05.1890. INV.NO.1107 (1 SUBM*, 1 JUV $^{*}$ ): Philodromus dispar Walck., Rijeka, 03.09.1897. INV.NO.2055 (1 F): Philodromus dispar Walck., Bakar, Damin. INV.NO.2055 (2 JUV*): Philodromus dispar Walck., Bakar, Damin. INV.NO.2381 (1 F): Philodromus aureolus rufolimbatus Clerck verus, Lukovo, Sv. Juraj, Damin. 
Philodromus emarginatus (Schrank, 1803)

INV.NO.859 (1 F, 1 SUBM*): Philodromus emarginatus Schrk., Lokrum, Damin.

Philodromus fuscolimbatus Lucas, 1846

INV.NO.1050 (1 F): Philodromus aureolus Clerck verus. INV.NO.973 (2 JUV*): Philodromus aureolus rufolimbatus Clerck verus, Križišće, 12.08.1893., Damin. INV. NO.1084 (1 JUV*): Philodromus aureolus rufolimbatus Clerck verus, Lukovo, 09.07.1899. INV.NO.1094 (2 JUV*): Philodromus aureolus rufolimbatus Clerck verus. INV.NO.1703 $\left(1 \mathrm{JUV}^{*}\right)$ : Philodromus aureolus rufolimbatus Clerck verus, Bribir-Novi, 15.04.1897. INV. NO.2382 (3 SUBM* ${ }^{*}$ 6JUV*): Philodromus aureolus rufolimbatus Clerck verus, Lukovo, Sv. Juraj, Damin.

Philodromus lividus Simon, 1875

INV.NO.862 (1 F): Philodromus lividus Sim.

Philodromus longipalpis Simon, 1870

INV.NO.966 (1 F): Philodromus aureolus Clerck verus, Lukovo, 28.08., Damin. INV. NO.974 (2 JUV*): Philodromus aureolus pallens Clerck verus, Zemun, 1899., Damin. INV. NO.975 (1 F): Philodromus aureolus pallens Clerck verus, Povile, 09.08.1899., Damin. INV. NO.1078 (1 F, 1 M): Philodromus aureolus pallens Clerck verus, Bakar, 1891. INV.NO.1099 (2 F): Philodromus aureolus Clerck verus, 17.08.1892.

Philodromus lunatus Muster \& Thaler, 2004

INV.NO.972 (2 F): Philodromus aureolus rufolimbatus Clerck verus, Lukovo, Sv. Juraj, Damin.

Philodromus margaritatus (Clerck, 1757)***

INV.NO.961 (1 JUV*): Philodromus margaritatus Clerck., Pod Bitorajem, 26.6.1892., Damin. INV.NO.963 (3SUBF*, 3 JUV*): Philodromus margaritatus Clerck., Osijek. INV. NO.963 (4 SUBM ${ }^{*}$ ): Philodromus margaritatus Clerck., Osijek. INV.NO.1090 (1 SUBF*, 1 SUBM $^{*}$ ): Philodromus margaritatus Clerck., Plitvice, 27.8.1902. INV.NO.2036 (1 SUBF*): Philodromus margaritatus Clerck.

Philodromus marmoratus Kulczyński, 1891***

INV.NO.971 (1 SUBM*): Philodromus aureolus marmorata Clerck verus, Bribir, Damin.

Philodromus poecilus (Thorell, 1872)

INV.NO.857 (1 M): Philodromus poecillus Thor., Osijek, Damin. INV.NO.857 (1 F): Philodromus poecillus Thor., Osijek, Damin. INV.NO.858 (3 JUV*): Philodromus poecillus Thor., Hvar, Damin. INV.NO.962 (1 M): Philodromus margaritatus Clerck., Božjakovina, Dr. Langhoffer. INV.NO.963 (1 F): Philodromus margaritatus Clerck., Osijek. INV.NO.1073 (1 SUBM* $2 \mathrm{JUV}^{*}$ ): Philodromus poecillus Thor., Plitvice, 27.8.1902. INV.NO.1101 (1 F, $1 \mathrm{JUV}^{*}$ ): Philodromus poecillus Thor., Kecskemet. INV.NO.1957 (5 F, 2 M): Philodromus margaritatus Clerck., Zemun, 1894.

Philodromus rufus Walckenaer, 1826

INV.NO.1083 (1 JUV*): Philodromus rufus Walck., Topusko, 8.1891. INV.NO.1096 (1 F): Philodromus rufus Walck. INV.NO.1108 (1 F): Philodromus rufus Walck. INV.NO.1109 (1 F): Philodromus rufus Walck., Bakar, 10.1891. 
Philodromus sp.

INV.NO.1074 (1 SUBF*): Philodromus sp., Fužine, 6.1897. INV.NO.1077 (2 JUV): Philodromus sp., Topusko, 8.1891. INV.NO.1079 (1 JUV): Philodromus sp., 1901., Z. Bučar. INV.NO.1085 (2 JUV): Philodromus sp. INV.NO.1086 (4 JUV): Philodromus sp., INV.NO.1091 (5 JUV): Philodromus sp., Bribir-Novi, 12.9.1897. INV.NO.1110 (1 JUV): Philodromus sp., Osijek, 1897., Dr. Langhoffer. INV.NO.1111 (1 JUV): Philodromus sp., Bribir-Novi, 15.4.1897. INV.NO.1293 (1 SUBM): Linyphia marginata C.L. Koch, Topusko, 8.1901. INV.NO.2345 (1 JUV): Mimetus laevigatus Keys., Porto Palazzo. INV.NO.2409 (1 JUV): Theridium lineatum Clerck., Arbanasi.

Thanatus arenarius L. Koch, 1872

INV.NO.891 (1 F): Thanatus arenarius Thor., Šibenik, 8.1891., Damin N. INV.NO.892 (2 $\mathrm{JUV}^{*}$ ): Thanatus arenarius Thor., Osijek.

Thanatus atratus Simon, 1875

INV.NO.894 (1 F, 1 M): Thanatus vulgaris E. Sim., ? Senj.

Thanatus coloradensis Keyserling, 1880***

INV.NO.1097 (1 F): Thanatus sp., Senj, 06.07.1899.

Thanatus formicinus (Clerck, 1757)

INV.NO.890 (1 F): Thanatus formicinus Clerck.

Thanatus sp.

INV.NO.898 (1 JUV): Heriaeus Savignyi E. Sim., 8.8.1894. INV.NO.1102 (1 JUV): Thanatus sp., Omišalj.

Thanatus vulgaris Simon, 1870

INV.NO.893 (2 F, 19 JUV*): Thanatus vulgaris E. Sim., ? Bakar.

Tibellus macellus Simon, 1875

INV.NO.810 (4 F, 2 M, 2 SUBM*, 3 JUV*): Tibellus vittatus Thor., Bakar, 5.6.1897., Damin. INV.NO.811 (1 M): Tibellus vittatus Thor., Rijeka, 8.6.1897., Langhoffer. INV.NO.811 (1 F): Tibellus vittatus Thor., Rijeka, 8.6.1897., Langhoffer. INV.NO.1801 (12 JUV*): Tibellus vittatus Thor., Bakar, Langhoffer.

Tibellus oblongus (Walckenaer, 1802)

INV.NO.812 (1 M): Tibellus parallelus C.L. Koch, Božjakovina, Langhoffer. INV. NO.1092 (1 F): Tibellus parallelus C.L. Koch, Zemun, 1899., Canjuga. INV.NO.1816 (1 F, 1 JUV): Tibellus macellus Simon, Viljevo, 18.08.1915. INV.NO.2100 (1 F): Tibellus oblongus Walck., Kolan, 26.07.1956. INV.NO.2110 (1 F): Tibellus sp., Caska, 09.06.1958.

Tibellus sp.

INV.NO.1075 (1 JUV): Tibellus sp., Križevci, 4.9.1897. INV.NO.1080 (1 JUV): Tibellus sp., Rijeka, 2.9.1897. INV.NO.1274 (1 JUV): Steatoda bipunctata L. Koch, Osijek, 1907.

\section{Pholcidae C. L. Koch, 1850}

Holocnemus pluchei (Scopoli, 1763)

INV.NO.367 (4 F, 2 M): Holocnemus rivulatus Forsk. INV.NO.368 (2 M, 2 JUV*): Holocnemus rivulatus Forsk. INV.NO.1445 (1 M): Pholcus phalangioides Fuessly, Damin 
N. INV.NO.2008 (3 F): Pholcus opilionides Schr., Buk Krke. INV.NO.2287 (1 F): Holocnemus Forskalii Thor., Zemun, 1899., Damin N. INV.NO.2396 (1 F, 1 M): Pholcus phalangioides Fuessly, Damin N.

Holocnemus sp.

INV.NO.1445 (1 JUV): Pholcus phalangioides Fuessly, Damin N. INV.NO.2008 (5 JUV, 1 SUBM): Pholcus opilionides Schr., Buk Krke.

Hoplopholcus forskali (Thorell, 1871)

INV.NO.290 (24 F, 17 M, 28JUV*): Holocnemus Forskalii Thor., Zemun, 1899., Damin N. INV.NO.1805 (1 M): Holocnemus Forskalii Thor., Solin. INV.NO.2007 (1 JUV*): Holocnemus Forskalii Thor., Osijek. INV.NO.2382 (1 M): Steatoda bipunctata L., Zemun, 1899., Damin N.

Pholcus opilionoides (Schrank, 1781)

INV.NO.1177 (2 F): Pholcus opilionides Schr., Topusko, 08.1891. INV.NO.1443 (1 F): Pholcus opilionides Schr., Jelenje, Damin N. INV.NO.2004 (1 F): Pholcus phalangioides Fuessly, Izvor Rječine, 27.05.

Pholcus phalangioides (Fuesslin, 1775)

INV.NO.1444 (1 F, 1 M, 1 JUV*): Pholcus phalangioides Fuessly, Rijeka (Fiume), Damin N. INV.NO.1446 (1 M, 3 JUV*): Pholcus phalangioides Fuessly, Damin N. N. INV.NO.1447 (3 F): Pholcus phalangioides Fuessly, Zemun, Damin N. INV.NO.1448 (1 F, 3 M, 1 JUV*): Pholcus phalangioides Fuessly, Pećine (Vrana), Damin. INV.NO.2012 (1 F): Pholcus opilionides Schr., Kalnik, 4.6.1917. INV.NO.2017 (2 F): Pholcus opilionides Schr., Zagreb, 08.12 INV.NO.2295 (1 M): Holocnemus rivulatus Forsk. INV.NO.2296 (2 F): Holocnemus rivulatus Forsk. INV.NO.2408 (1 M): Epeira Redii Scop., Bag.

Pholcus sp.

INV.NO.2286 (1 JUV*): Holocnemus Forskalii Thor., Zemun, 1899., Damin N.

Psilochorus sp.

INV.NO.1182 (1 JUV): Holocnemus Forskalii Thor., Osijek, 12.1898.

Spermophora senoculata (Dugès, 1836)

INV.NO.269 (3 F, 1 JUV*): Spermophora senoculata Dug., Primorje cijelo.

\section{Phrurolithidae Banks, 1892}

Phrurolithus festivus (C. L. Koch, 1835)

INV.NO.2068 (1 F, 2 M, 2SUBM*): Phrurolithus festivus C.L. Koch.

Phrurolithus sp.

INV.NO.1422 (1 JUV): Phrurolithus sp. ? Grižane, 14.04.1900.

\section{Pisauridae Simon, 1890}

Dolomedes fimbriatus (Clerck, 1757)

INV.NO.1792 (1 F): Dolomedes fimbriatus Clerck., Dubrava kod Sesveta, 15.05.1915., Škorić. INV.NO.685 (1 JUV*): Dolomedes fimbriatus Clerck., Osijek, Damin. INV.NO.1748 
(2 JUV*): Dolomedes fimbriatus Clerck., Unčica, 29.08.1911. INV.NO.1749 (1 JUV*): Dolomedes fimbriatus Clerck., Zagreb.- vrt, 02.06.1903., Polić. INV.NO.1757 (1 JUV*): Dolomedes fimbriatus Clerck., Mošćenice, 01.09.1911. INV.NO.1771 (3 JUV*): Dolomedes fimbriatus Clerck., Mošćenice, 01.09.1911. INV.NO.1775 (1 JUV*): Dolomedes fimbriatus Clerck., Babina greda, 17.07.1912. INV.NO.1776 (1 JUV*): Dolomedes fimbriatus Clerck., Božjakovina, 30.09.1910. INV.NO.1793 (1 JUV*): Dolomedes fimbriatus Clerck., Batum, 07.09.1903. INV.NO.1846 (2 JUV*): Dolomedes limbatus Hahn., Viljevo, 18.08.1915.

Dolomedes plantarius (Clerck, 1757)

INV.NO.686 (1 JUV*): Dolomedes plantarius CI., Božjakovina, Langhoffer.

Dolomedes sp.

INV.NO.344 (1 JUV): Dolomedes sp., Ozalj pred spiljom, 16.7.1902. INV.NO.635 (1 JUV): Dolomedes sp., Križevci, 4.7.1897. INV.NO.651 (1 JUV): Dolomedes sp., Zagreb, 1898. INV. NO.703 (1 JUV): Dolomedes sp., Bregi, 13.7.1902. INV.NO.734 (2 JUV): Dolomedes sp., Topusko, 8.1891.

\section{Pisaura mirabilis (Clerck, 1757)}

INV.NO.379 (1 M): Pisaura mirabilis CI., Rijeka, 09.0mj., Damin. INV.NO.380 (1 M, 2 JUV$\left.^{*}\right)$ : Pisaura mirabilis CI. INV.NO.381 (4 F, $\left.1 \mathrm{M}, 9 \mathrm{JUV}^{*}\right)$ : Pisaura mirabilis CI. INV. NO.519 (4 JUV ${ }^{*}$ : Pisaura mirabilis CI., Osijek, 12.1898. INV.NO.563 (1 JUV*): Pisaura mirabilis CI., Malinska, 27.3. INV.NO.1558 (1 M): Pisaura mirabilis CI., Osijek, 1897., Dr. Langhoffer. INV.NO.1600 (1 F): Pisaura mirabilis CI., Vrata, 22.7. INV.NO.1772 (1 M): Pisaura mirabilis CI., Fruška gora, 30.05.1918. INV.NO.1783 (1 M): Pisaura mirabilis CI., Bakar, 29.03.1893. INV.NO.1785 (2 F, 1 M): Pisaura mirabilis CI., Zemun, 1899. INV. NO.1791 (1 F): Pisaura mirabilis CI., Zagreb, 20.05.1903. INV.NO.1795 (1 F): Pisaura mirabilis CI., Brešce, 03.06.1903., Polić. INV.NO.1841 (1 F): Pisaura mirabilis CI., Tuškanac, 19.05.1905. INV.NO.1858 (1 M): Dolomedes fimbriatus Clerck., Maksimir, 20.04.1916. INV. NO.2099 (1 F): Pisaura listeri Scop., o. Pag, Kolansko blato, 27.06.1960. INV.NO.2165 (1 F): Pisaura listeri Scop., Caska, o. Pag, 25.06.1960. INV.NO.2198 (1 F): Pisaura listeri Scop., Unije, 29.06.1963. INV.NO.613 (3 JUV*): Pisaura mirabilis CI., Križevci, 4.9.1897. INV. NO.614 (2 JUV*): Pisaura mirabilis CI., 1901., Trgovčević. INV.NO.618 (1 JUV*): Pisaura mirabilis CI., Starigrad, 29.7. INV.NO.676 (1 JUV*): Pisaura mirabilis CI., Zagreb, Kraljičin zdenac, 12.4.1902. INV.NO.733 (1 JUV*): Pisaura mirabilis CI., Kostrena Sv. Barb., 2.5.1900. INV.NO.775 (8JUV*): Pisaura mirabilis CI., Osijek, 1907., Dr. Langhoffer. INV.NO.776 (5 $\left.\mathrm{JUV}^{*}\right)$ : Pisaura mirabilis CI. INV.NO.1024 (1 JUV*): Pisaura mirabilis CI. INV.NO.1563 (2 $J_{U V}^{*}$ ): Pisaura mirabilis CI., Zagreb, 1897., Dr. Langhoffer. INV.NO.1586 (2 JUV*): Pisaura mirabilis CI., Kraljičin zdenac, 18.9.1901. INV.NO.1723 (1 JUV*): Pisaura mirabilis CI., Kraljičin zdenac, 17.07.1897., Langhoffer. INV.NO.1728 (1 JUV*): Pisaura mirabilis CI., Vrbica. INV.NO.1746 (1 JUV*): Pisaura mirabilis CI., Plitvice, 26.08.1902. INV.NO.1747 (1 JUV*): Pisaura mirabilis CI., Unčica, 28.08.1911. INV.NO.1777 (1 JUV*): Pisaura mirabilis CI., Sljeme, 10.09.1905., Poljugan. INV.NO.1778 (1 JUV*): Pisaura mirabilis CI., Zdenčina, 20.08.1912. INV.NO.1779 (1 JUV*): Pisaura mirabilis CI., Zdenčina, 20.08.1912. INV. NO.1783 (1 JUV*): Pisaura mirabilis CI., Bakar, 29.03.1893. INV.NO.1790 (1 JUV*): Pisaura mirabilis CI., Vrgovac?, 02.01.1915. INV.NO.1791 (1 SUBM*): Pisaura mirabilis CI., Zagreb, 20.05.1903.

\section{Pisaura sp.}

INV.NO.1858 (1 SUBM): Dolomedes fimbriatus Clerck., Maksimir, 20.04.1916. INV. NO.2351 (1 JUV): Anyphaena accentuata Walck., Bakar, 12.04.1892., Damin. INV.NO.2401 (1 JUV): Epeira Redii Scop., Novi, 08.04.1898. 


\section{Salticidae Blackwall, 1841}

Aelurillus v-insignitus (Clerck, 1757)

INV.NO.57 (1 F): Aelurillus v-insignitus Clerck., Skradin, Damin. INV.NO.58 (2 M): Aelurillus v-insignitus Clerck., Senj, Damin. INV.NO.1928 (2 F): Philaeus bicolor Walck., Senj.

Asianellus festivus (C. L. Koch, 1834)

INV.NO.60 (1 M): Aelurillus gilvus E. Sim.

Ballus chalybeius (Walckenaer, 1802)

INV.NO.6 (3 F, 3 M): Ballus depressus Walck., Izvor Rječine, 22.05.1890., Damin. INV. NO.6 (): Ballus depressus Walck., Izvor Rječine, 22.05.1890., Damin. INV.NO.7 (3 F, 2 M, $1 \mathrm{JUV}^{*}$ ): Ballus depressus Walck., Lovran, Damin. INV.NO.9 (9 F, 5 M, 2 SUBM*, 1 JUV $^{*}$ ): Ballus depressus Walck., Kotor, Damin. INV.NO.309 (1 JUV*): Ballus depressus Walck., Bribir-Novi, 15.4.1897. INV.NO.319 (1 JUV*): Ballus depressus Walck., Topusko, 8.1891. INV.NO.456 (1 M): Drassodes minusculus (=Dalmat) L. Koch. INV.NO.1917 (1 M): Attus sp., Zagreb (vrt), 25.04.1903.

Bryantella cf. smaragdus (Crane, 1945)

INV.NO.2433 (2 M): Heliophanus cupreus Walck., Juž. Amerika, 1905.

Carrhotus xanthogramma (Latreille, 1819)

INV.NO.1062 (1 JUV*): Philaeus bicolor Walck., Rijeka, Damin. INV.NO.1064 (2 JUV*): Philaeus bicolor Walck., Jablanac, 11.7., Damin. INV.NO.1065 (4 JUV*): Philaeus bicolor Walck., Skradin, Damin. INV.NO.1066 (4 JUV*): Philaeus bicolor Walck., Zadar, Damin. INV.NO.1067 (4 JUV*): Philaeus bicolor Walck., Porto Palazzo. INV.NO.1548 (1 SUBF*): Philaeus bicolor Walck., Osijek, 1897., Langhoffer. INV.NO.1560 (5 JUV*): Philaeus bicolor Walck., Topusko, 8.1891. INV.NO.1564 (4 JUV*): Philaeus bicolor Walck., Grižane, 14.4. INV.NO.1593 (2 JUV*): Philaeus bicolor Walck., Rijeka, 1.10.1901. INV.NO.1601 (1 M): Philaeus bicolor Walck., Kecskemet, 3.1890. INV.NO.1733 (2 F, 3 M): Philaeus bicolor Walck., Đakovo. INV.NO.1909 (3 JUV*): Philaeus bicolor Walck., Solin. INV.NO.1910 (2 JUV*): Philaeus bicolor Walck., Pad Krke. INV.NO.1924 (5 JUV*): Philaeus bicolor Walck., Buk Krke. INV.NO.1927 (4 SUBF* 4 JUV*): Philaeus bicolor Walck., Bakar. INV.NO.1928 $\left(2 \mathrm{JUV}^{*}\right)$ : Philaeus bicolor Walck., Senj. INV.NO.2025 (9JUV*): Philaeus bicolor Walck., Sv. Kuzam-Bakar, 14.08. INV.NO.2038 (1 JUV*, 5M): Philaeus bicolor Walck.

Chalcoscirtus infimus (Simon, 1868)

INV.NO.324 (1 M): Chalcoscirtus infimus E. Sim., Bakar, Damin. INV.NO.325 (1 F): Chalcoscirtus infimus E. Sim., Bag, Damin. INV.NO.2035 (2 F): Chalcoscirtus infimus E. Sim.,

Cyrba algerina (Lucas, 1846)***

INV.NO.168 (2 JUV*): Cyrba algerina ILuc., Sv. Duh, o. Krk.

Dendryphantes rudis (Sundevall, 1833)*

INV.NO.52 (2 F, 4 JUV*): Dendryphantes rudis Sund., Fužine, 22.08.1889., Damin.

Euophrys frontalis (Walckenaer, 1802)

INV.NO.194 (3 F, 1 SUBF*, 4 JUV*): Euophrys frontalis Walck., Zadar, Damin. INV. NO.194 (): Euophrys frontalis Walck., Zadar, Damin. INV.NO.194 (): Euophrys frontalis Walck., Zadar, Damin. INV.NO.1686 (1 M): Euophrys rufibarbis E. Sim., Bakar. 


\section{Euophrys rufibarbis (Simon, 1868)}

INV.NO.158 (1 JUV*): Euophrys rufibarbis E. Simon, Kraljevica, 31.07.1899., Damin. INV.NO.307 (3 JUV*): Euophrys rufibarbis E. Sim., Stari Grad, 11.7.1899. INV.NO.334 (1 SUBM $^{*}$ ): Euophrys rufibarbis E. Sim., Jablanac, 11.07., Damin. INV.NO.335 (1 F): Euophrys rufibarbis E. Sim., Dalmacija, Damin. INV.NO.337 (10 F, $\left.1 \mathrm{JUV}^{*}\right)$ : Euophrys rufibarbis E. Sim., Kraljevica, 8.7., Damin. INV.NO.339 (12 F, 4M, 6JUV*): Euophrys rufibarbis, BakarKrasina, 10.9.1890., Damin. INV.NO.1686 (1 SUBM*, 4 JUV*): Euophrys rufibarbis E. Sim., Bakar. INV.NO.2290 (2 M): Euophrys imitata E. Sim., Kostrena, 22.4., Damin.

Euophrys sulphurea (L. Koch, 1867)

INV.NO.195 (1 M): Euophrys sulfurea L. Koch.

\section{Evarcha arcuata (Clerck, 1757)}

INV.NO.877 (7 M): Ergane arcuata Clerck. INV.NO.1556 (1 F, 3 M, 1 JUV*): Ergane arcuata Clerck., Zagreb, 1897. INV.NO.1578 (4M): Callilepis exornata C.L. Koch, Bakar, 10.08.1900. INV.NO.1580 (2SSP*): Ergane arcuata Clerck., Jasikovac, 18.08.1902. INV. NO.1582 (1 M): Ergane arcuata Clerck., Križevci, 04.09.1898. INV.NO.1599 (1 SUBM*): Ergane arcuata Clerck., Zagreb, 1898.

Evarcha falcata (Clerck, 1757)

INV.NO.876 (6 F, 5 M, 1 SUBF*, 3 SUBM*): Ergane falcata Clerck., Bitoraj, 26.06.1892., Damin. INV.NO.879 (1 M): Ergane falcata Clerck., Zagreb. gora, Langhoffer. INV.NO.879 (1 F): Ergane falcata Clerck., Zagreb. gora, Langhoffer. INV.NO.880 (1 SUBF*): Ergane falcata Clerck., Gasperini. INV.NO.881 (1 M): Ergane falcata Clerck., Mrzla vodica, 18.08., Damin. INV.NO.1553 (1 JUV*): Ergane falcata Clerck., Praprad, 27.07.1900. INV.NO.1555 (1 M): Ergane falcata Clerck., Kralj. Zdenac (Zgrb), 14.06.1897., Dr. Langhoffer. INV.NO.1588 (1 F): Ergane falcata Clerck. INV.NO.1589 (1 F): Ergane falcata Clerck., Sljeme, 01.06.1898. INV.NO.1595 (2 F): Ergane falcata Clerck., Zagreb, 1897., Dr. Langhoffer. INV.NO.1918 (2 M): Ergane falcata Clerck., Bitoraj.

Evarcha jucunda (Lucas, 1846)

INV.NO.870 (1 M): Ergane iucunda Luc.Kotor, 03.08.1894., Damin. INV.NO.871 (3 F, 3 M5 JUV*): Ergane iucunda Luc., Gasperini. INV.NO.871 (): Ergane iucunda Luc., Gasperini. INV.NO.871 (): Ergane iucunda Luc., Gasperini. INV.NO.872 (1 M): Ergane iucunda Luc., Lokrum, 07.08.1894., Damin. INV.NO.872 (3 JUV*): Ergane iucunda Luc., Lokrum, 07.08.1894., Damin. INV.NO.873 (3 F, 1 M): Ergane iucunda Luc., Jablanac, 11.07.1899.,

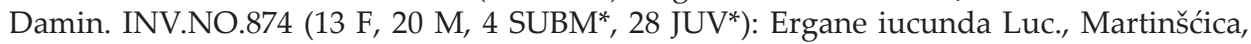
14.08.1890., Damin. INV.NO.875 (1 M): Ergane iucunda Luc., Vis. INV.NO.1561 (1 F): Ergane iucunda Luc., Starigrad, 29.08. INV.NO.1573 (1 M): Ergane iucunda Luc., Skradin. INV. NO.1574 (1 JUV*): Ergane iucunda Luc., Bribir-Novi, 15.04.1897. INV.NO.1584 (2 JUV*): Ergane iucunda Luc., Borova draga, 08.07.1897. INV.NO.1592*(1 F): Ergane iucunda Luc., Buk Krke. INV.NO.1724 (2 JUV*): Ergane iucunda, Bribir-Novi, 15.04.1897. INV.NO.1741 (2 F, 3 M, 7 JUV*): Ergane iucunda Luc., Bakar. INV.NO.2442 (1 M): Epeira adianta Walck., Caska, o. Pag, 28.06.1960.

Evarcha laetabunda (C. L. Koch, 1846)

INV.NO.878 (1 F, 1 M): Ergane laetabunda C.L. Koch.

Evarchasp.

INV.NO.1578 (2SUBM): Callilepis exornata C.L. Koch, Bakar, 10.08.1900. 
Frigga cf. crocuta (Taczanowski, 1879)

INV.NO.1929 (2 M): Heliophanus cupreus Walck., Juž. Amerika, 1905.

Heliophanus auratus C. L. Koch, 1835

INV.NO.3 (1 F): Heliophanus auratus C.L. Koch, Podsused, 25.05.1906. INV.NO.120 (1 F): Heliophanus exsultans E. Sim., Bitoraj-podnožje, 26.06.1892. INV.NO.121 (1 F): Heliophanus auratus C.L. Koch, Bregi, 13.07.1902. INV.NO.593 (5 F, 2 M): Heliophanus auratus C.L. Koch. Bakarac, Damin. INV.NO.603 (1 M): Heliophanus exsultans E. Sim., Split, Damin. INV.NO.1925 (3 F): Heliophanus cupreus Walck., Podnožje Bitoraja. INV.NO.2320 (1 F): Heliophanus lineiventris E. Sim., Gasperini. INV.NO.2432 (6 F, 2 M): Heliophanus cupreus Walck., lzvor Rječine, 22.05.1897. INV.NO.120 (4 JUV*): Heliophanus exsultans E. Sim., Bitoraj-podnožje, 26.06.1892. INV.NO.605 (1SSP*): Heliophanus exsultans E. Sim., Damin.

Heliophanus cupreus (Walckenaer, 1802)

INV.NO.4 (1 SUBF*): Heliophanus cupreus Walck., Jasikovac, 18.08.1902. INV.NO.33 (1 F): Heliophanus sp. Rijeka, 23.09.1897. INV.NO.47 (1 F): Heliophanus cupreus Walck. INV.NO.55 (1 F): Heliophanus cupreus Walck., Križevci, 14.09.1897. INV.NO.69 (1 F): Heliophanus cupreus Walck., Bribir-Novi, 15.04.1897. INV.NO.96 (1 F): Heliophanus cupreus Walck., Fužine, 06.06.1897. INV.NO.586 (10 M, 15 F): Heliophanus cupreus Walck., Bakar, Damin. INV.NO.815 (1 M, 2 F): Leptorchestes berolinensis C.L. Koch, Zagrebač. gora, Langhoffer. INV.NO.1906 (1 F): Heliophanus cupreus Walck., Bakar. INV.NO.1912 (1 F): Attus sp., Beočin, 29.05.1910. INV.NO.1919 (3 M, 3 F): Heliophanus cupreus Walck., lzvor Rječine, 22.05.1897. INV.NO.1925 (1 SUBF*): Heliophanus cupreus Walck., Podnožje Bitoraja. INV.NO.1931 (1 F): Attus sp., Vrdnik, 28.07.1917. INV.NO.2429 (10 JUV*): Heliophanus cupreus Walck., Bakar. INV.NO.2430 (3 SUBM ${ }^{*}$ ): Heliophanus cupreus Walck., Bakar. INV.NO.585 (1 SUBF*, 2 JUV*): Heliophanus cupreus Walck., Križišće, 16.8.1893., Damin. INV.NO.586 (1 JUV*): Heliophanus cupreus Walck., Bakar, Damin. INV. NO.2319 (1 JUV*): Heliophanus cupreus Walck., Križišće, 16.8.1893., Damin.

Heliophanus equester L. Koch, 1867

INV.NO.1683 (1 F): Heliophanus sp., Zadar. INV.NO.1922 (1 F): Heliophanus equester L. Koch, Pad Krke.

Heliophanus flavipes (Hahn, 1832)

INV.NO.11 (1 F): Heliophanus flavipes Hahn., Fužine, 06.1897. INV.NO.594 (1 M, 3 F ): Heliophanus flavipes Hahn., Gasperini. INV.NO.595 (1 SUBF*): Heliophanus flavipes Hahn., Gasperini.

\section{Heliophanus kochii Simon, 1868}

INV.NO.5 (1 SUBM*): Heliophanus Koch, ii E. Simon, Stari grad, 11.07.1899. INV. NO.12 (1 SUBM*): Heliophanus Koch, ii E. Simon, Stari grad, 17.09. INV.NO.32 (2 F): Heliophanus Koch, ii E. Simon, Novi, 19.07. INV.NO.49 (1 F , 1 SUBM ${ }^{*}$ ): Heliophanus Koch, ii E. Simon, Grižane, 6.8.1891. INV.NO.59 (1 M): Heliophanus Koch, ii E. Simon, Bribir-Novi, 15.04.1897. INV.NO.90 (1 M): Heliophanus Koch, ii E. Simon, Novi, 25.09.1901. INV.NO.97 (1 F): Heliophanus Koch, ii E. Simon, Zadar, 15.07.1899. INV.NO.123 (3 F , 1 $J_{U V}^{*}$ ): Heliophanus Koch, ii E. Simon, Skradin, 23.07.1894. INV.NO.146 (1 F): Heliophanus Koch, ii E. Simon, Bakar. INV.NO.587 (1 F): Heliophanus Koch, ii E. Sim., Senj, 6.7., Damin. INV.NO.588 (9 F, 4 SUBM*, 4 M, 2 JUV*, 4 SUBM*): Heliophanus Koch, ii E. Sim., Bakar, Damin. INV.NO.589 (1 M, $\left.1 \mathrm{JUV}^{*}\right)$ : Heliophanus Koch, ii E. Sim., Cavtat, Damin. INV. 
NO.590 (1 JUV*, 1 SUBM*): Heliophanus Koch, ii E. Sim., Bag, 14.7., Damin. INV.NO.608 (4 F): Heliophanus cupreus Walck. INV.NO.813 (2 F): Leptorchestes berolinensis C.L. Koch, Mljet (Polača). INV.NO.1900 (1 F, 3 JUV*): Heliophanus Koch, ii E. Sim., Buk Krke. INV. NO.1901 (3 F, 1 SUBM*, 1 M, 5 JUV*): Heliophanus Koch, ii E. Sim., Bakar. INV.NO.1903 (1 F): Heliophanus cupreus Walck., Ušće Raše, 31.05.1896. INV.NO.1914 (2 M, 2 SUBM*, 3 JUV*): Heliophanus Koch, ii E. Sim., Senj. INV.NO.2320 (1 M): Heliophanus Cambridgei E. Sim., Gasperini. INV.NO.2387 (1 F): Crustulina scabripes E. Sim., Novi, 19.7. INV.NO.2431 (1 F): Heliophanus cupreus Walck., Bakar.

\section{Heliophanus melinus L. Koch, 1867}

INV.NO.26 (2 F): Heliophanus melinus L. Koch. Borova draga, 06.07. INV.NO.591 (2 M): Heliophanus lineiventris E. Sim., Kotor, Damin. INV.NO.592 (1 M): Heliophanus lineiventris E. Sim., Gasperini. INV.NO.597 (4M, 1 SUBF, 1 SUBM, 1SSP): Heliophanus melinus L. Koch, Bakar, Damin. INV.NO.598 (1 F): Heliophanus melinus L. Koch, Tajer, Damin. INV.NO.599 (2 F, 1 SUBF*): Heliophanus melinus L. Koch, Crikvenica-Voz. B., Damin. INV.NO.600 (1 SUBM*): Heliophanus melinus L. Koch, Gasperini. INV.NO.601 (1 M): Heliophanus viriatus. INV.NO.606 (1 F): Heliophanus Cambridgei E. Sim., Gasperini. INV.NO.1552 (1 F): Heliophanus melinus L. Koch, Lukovo, 9.7.

Heliophanus patagiatus Thorell, 1875

INV.NO.1732 (1 M): Heliophanus patagiatus Thor., Bosna, Apfelbeck.

Heliophanus simplex Simon, $1868^{* * *}$

INV.NO.584 (2 SUBM*, 1 JUV*, 1SSP*): Heliophanus simplex E. Sim., Kraljevica, Damin.

Heliophanus sp.

INV.NO.2359 (1 JUV): Leptorchestes berolinensis C.L. Koch, Mljet (Polača).

Heliophanus tribulosus Simon, 1868

INV.NO.595(3F): Heliophanus flavipes Hahn., Gasperini. INV.NO.596(2F):Heliophanus flavipes Hahn., Kotor, Muo, Damin. INV.NO.602 (1 F): Heliophanus cambridgei E. Sim., Cavtat, Damin. INV.NO.1062 (1 M): Philaeus bicolor Walck., Rijeka, Damin. INV.NO.2064 (1 M): Heliophanus Cambridgei E. Sim., INV.NO.2428 (1 M): Heliophanus cupreus Walck., Bakar. INV.NO.604 (1 SUBF*): Heliophanus cambridgei E. Sim., Bakar, 14.7., Damin. INV. NO.607 (1 SUBM* 2 JUV*): Heliophanus cambridgei E. Sim., Porto Palazzo, Damin.

Icius hamatus (C. L. Koch, 1846)

INV.NO.100 (1 F): Icius notabilis C.L. Koch, Split, Vranica, Damin. INV.NO.101 (2 F): Icius notabilis C.L. Koch, Gasperini. INV.NO.99 (1 JUV*): Icius striatus Walck., Košljun, Damin.

Icius sp.

INV.NO.2251 (1 JUV): Enoplognatha mandibularis Luc., Split, Damin.

Leptorchestes berolinensis (C. L. Koch, 1846)

INV.NO.814 (1 F): Leptorchestes berolinensis C.L. Koch, Prevez (Raša), Damin. INV. NO.816 (1 F, 1 M, 1 SUBM*): Leptorchestes berolinensis C.L. Koch, Rijeka, Damin.

Macaroeris nidicolens (Walckenaer, 1802)

INV.NO.21 (1 F, 1 JUV*): Dendryphantes nidicolens Walck., 01.1901., Z. Bučar. INV. NO.22 (1 M): Dendryphantes nidicolens Walck., Bakar, Damin. INV.NO.22 (1 F, 5 JUV*): 
Dendryphantes nidicolens Walck., Bakar, Damin. INV.NO.23 (1 F): Dendryphantes nidicolens Walck., Vrbica, Damin. INV.NO.24 (1 F, 1 M, $\left.24 \mathrm{JUV}^{*}\right)$ : Dendryphantes nidicolens Walck., Bakar, Damin. INV.NO.44 (1 JUV*, $1 \mathrm{M})$ : Dendryphantes nidicolens Walck., Fužine, 06.06.1897. INV.NO.46 (1 SSP*, 1 F, 1 JUV*): Dendryphantes nidicolens Walck., Gasperini. INV.NO.51 (1 F, $\left.1 \mathrm{JUV}^{*}\right)$ : Dendryphantes nidicolens Walck., Borova draga, 08.07.1897. INV.NO.98 (2 JUV*): Dendryphantes nidicolens Walck., Grad Mali. INV. NO.105 (2 JUV*): Dendryphantes nidicolens Walck., Bribir-Novi, 15.04.1897. INV.NO.1739 (2 F , $1 \mathrm{JUV}^{*}$ ): Dendryphantes nidicolens Walck., Crikvenica, 12.08.

\section{Marpissa muscosa (Clerck, 1757)}

INV.NO.91 (1 F): Marpissa muscosa Clerck., Osijek, 07.1901., Rössler. INV.NO.151 (1 F): Marptusa muscosa Clerck., Zagreb, Sofijin put, 30.10.1898. INV.NO.152 (1 JUV*): Marptusa muscosa Clerck., Osijek, 12.1898. INV.NO.886 (1 F): Marptusa muscosa Clerck., Fužine, Damin. INV.NO.887 (4 F, 1 M): Marptusa muscosa Clerck., Osijek, Damin. INV.NO.1915 (1 F): Marptusa muscosa Clerck., Podsused. INV.NO.1930 (2 F): Marptusa muscosa Clerck., Osijek. INV.NO.1934 (1 F): Marptusa muscosa Clerck., Zagreb, 28.05.1903.

Marpissa pomatia (Walckenaer, 1802)

INV.NO.888 (1 JUV*): Marptusa pomatia Walck., Košljun, Krk, 30.3.1894., Damin. INV. NO.889 (1 F, 2 M): Marptusa pomatia Walck., Damin.

Marpissa radiata (Grube, 1859)

INV.NO.885 (2 F): Marptusa radiata Grube., Vrana (Dalm.), Damin.

Mendoza canestrinii (Ninni, 1868)*,***

INV.NO.50 (1 SUBF*): Hyctia canestrinii Can. et. Pav., Plitvice, 27.08.1902. INV.NO.64 (1 SUBF*): Hyctia canestrinii Can. et. Pav., Osijek, 1897., Langhoffer. INV.NO.1911 (1SSP*): Hyctia canestrinii Can. et. Pav., Starigrad, o. Hvar.

Menemerus semilimbatus (Hahn, 1829)

INV.NO.944 (8JUV*, 9SUBM*, 10M, 12 F): Menemerus semilimbatus Hahn., Gasperini. INV.NO.945 (1 F): Menemerus semilimbatus Hahn., Pećina (Vrana), 23.8., Damin. INV. NO.946 (4 F , 4M, 3 JUV*): Menemerus semilimbatus Hahn., Lovran, 7. mj., Damin. INV. NO.947 (2 F, 2 JUV*): Menemerus semilimbatus Hahn., Voz (Krk), 7.6.1891., Damin. INV. NO.948 (1 F, 1 M, $1 \mathrm{JUV}^{*}$ ): Menemerus semilimbatus Hahn., Stari grad, Damin. INV. NO.1738 (1 SUBM*): Menemerus semilimbatus Hahn., Bag, 14.07. INV.NO.1913 (1 JUV*): Menemerus semilimbatus Hahn., Lukovo, 17.07. INV.NO.1920 (1 F): Attus poubescens F., Split, 06.06.1909. INV.NO.1926 (1 F, 2 M): Menemerus semilimbatus Hahn., Bakar.

\section{Menemerus sp.}

INV.NO.67 (1 JUV*): Menemerus sp.

Myrmarachne formicaria (De Geer, 1778)

INV.NO.981 (1 M): Salticus formicarius Deg., Osijek, Damin. INV.NO.982 (2 F, 1 M, 3 $\left.J_{U V}^{*}\right)$ : Salticus formicarius Deg., Bakar, Damin. INV.NO.1591 (3 JUV*): Salticus formicarius Deg., Bakar, 1890.

Neaetha membrosa (Simon, 1868)

INV.NO.998 (1 F): Attus penicillatus E. Sim., Hundsberg. 
Neon pictus Kulczyński, 1891

INV.NO.328 (1 F): Neon pictus Kulcz., INV.NO.160\#: Neon pictus Kulcz., Malinska.

Neon reticulatus (Blackwall, 1853)

INV.NO.330 (1 JUV*): Neon reticulatus Blackw., Damin.

Pellenes brevis (Simon, 1868)

INV.NO.654 (1 F): Pellenes campylophorum, Damin.

Pellenes nigrociliatus (Simon, 1875)

INV.NO.653 (1 M, 3 JUV*): Pellenes Bedelii E. Sim., Bakar, Turčina, 1899., Damin. INV. NO.1916 (1 M, 1 JUV*): Pellenes Bedelii E. Sim., Senj.

Pellenes tripunctatus (Walckenaer, 1802)***

INV.NO.652 (2 SUBM*): Pellenes tripunctatus Walck., Vratnik, Damin.

Philaeus chrysops (Poda, 1761)

INV.NO.92 (1 JUV*): Philaeus chrysops Poda, Bakar. INV.NO.106 (2 JUV*): Philaeus chrysops Poda, Borova draga, 08.07. INV.NO.107 (1 F): Philaeus chrysops Poda, Kraljevica, 31.07.1899. INV.NO.153 (2 F): Philaeus chrysops Poda, Bag, 14.7.1899. INV.NO.157 (1 JUV*): Philaeus chrysops Poda, Grižane, 14.4.1900. INV.NO.1057 (2 F, 3 M): Philaeus chrysops Poda, Skradin, Damin. INV.NO.1058 (1 M): Philaeus chrysops Poda, Jelenje, 2.7., Damin. INV.NO.1059 (1 M, 1 F): Philaeus chrysops Poda, Lukovo, Damin. INV.NO.1060 (1 JUV*, $1 \mathrm{SUBM}^{*}$, $2 \mathrm{SUBF}^{*}$, 9 F, 16 M): Philaeus chrysops Poda, Primorje, Damin. INV.NO.1061 (4 SUBF*, 8 SUBM* 9 JUV*, 14 M): Philaeus chrysops Poda, Gasperini. INV.NO.1063 (1 F): Philaeus bicolor Walck., Izvor Rječine, 22.5., Damin. INV.NO.1726 (1 JUV*, 1 SUBM*): Philaeus chrysops var. bilineata Walck., 15.04.1897. INV.NO.1899 (1 M): Philaeus chrysops Poda, Buk Krke. INV.NO.1902 (1 M): Philaeus chrysops Poda, Ušće Raše, 31.05.1896. INV.NO.1921 (1 M): Philaeus chrysops Poda, Kalnik, 23.05.1917. INV.NO.1923 (1 JUV*, 2 M): Philaeus chrysops Poda, Bakar. INV.NO.1932 (1 M): Philaeus chrysops Poda, Senj, 05.06.1914. INV.NO.1933 (1 M): Philaeus chrysops Poda, Kotor, 01.06.1908. INV.NO.2095(1 F): Philaeus chrysops Poda, o. Pag, Kolansko blato, 27.06.1964. INV.NO.2129 (1M): Philaeus chrysops Poda, Barbat, 22.06.1960. INV.NO.2143 (1 M): Philaeus chrysops Poda, Caska, o. Pag, 26.06.1962. INV.NO.2229 (1 M): Philaeus bicolor Walck. INV.NO.2339 (1 F): Misumena tricuspidata Fabr., Skradin, Damin.

Philaeus sp.

INV.NO.305 (4 JUV): Philaeus sp.

Phintella castriesiana (Grube, 1861)

INV.NO.804 (2 JUV*): Maevia castresiana Grube. INV.NO.805 (1 JUV*, 2 SUBM $\left.^{*}\right)$ : Maevia castresiana Grube. INV.NO.1572 (1 F): Maevia castresiana Grube, Zagreb, 9.7.1902. INV.NO.1590 (1 JUV*): Maevia castresiana Grube., Topusko, 8.1891. INV.NO.2358 (1 JUV*): Maevia castresiana Grube.

Phlegra bresnieri (Lucas, 1846)

INV.NO.527 (1 F, 2 M): Oxiopes lineatus Latr., Gasperini. INV.NO.1217 (1 SUBM*): Phlegra Bresnieri Luc., Damin. INV.NO.1218 (1JUV*): Phlegra Bresnieri Luc., Zadar, Damin. INV.NO.1219 (1 M): Phlegra Bresnieri Luc., Gasperini. INV.NO.1223 (1 M): Phlegra lineata C. Koch, Split, Damin. 
Phlegra cinereofasciata (Simon, 1868)

INV.NO.1681 (1 M): Phlegra fuscipes Kulcz., Voz (kraj Kraljevice). INV.NO.1222 (1 $\mathrm{JUV}^{*}$ ): Phlegra fuscipes Kulcz., Kraljevica, 4.5.1891., Damin.

Phlegra fasciata (Hahn, 1826)

INV.NO.1220 (1 JUV*, 1 M): Phlegra fasciata Hahn., Bakar, Damin. INV.NO.1221 (1 JUV*): Phlegra fasciata Hahn., Karlobag, Damin. INV.NO.1712 (2 M): Phlegra sp., 03.10.1891.

Phlegra lineata (C. L. Koch, 1846)

INV.NO.1224 (1 F): Phlegra lineata C. Koch, Gasperini.

Plexippus petersi (Karsch, 1878)

INV.NO.1581 (1 F): Menemerus culicivorus, Senj.

Pseudeuophrys erratica (Walckenaer, 1826)

INV.NO.259 (1 M): Euophrys erratica Walck., Grižane, 4.4.1900. INV.NO.332 (1 M, 1 F): Euophrys erratica Walck., Rijeka, Damin. INV.NO.333 (2 JUV*): Euophrys erratica Walck., Karlobag, Lukovo, Damin. INV.NO.2277 (2 F): Euophrys vafra, Lukovo, 4.7.1899.

Pseudeuophrys obsoleta (Simon, 1868)

INV.NO.296 (2 JUV*, 2 F): Euophrys confusa Kulcz., Kraljevica, 31.7.1899. INV.NO.336 (1 JUV*, 5 F): Euophrys confusa Kulcz., Mandrač, Damin. INV.NO.338 (2 SUBM*, 3 JUV*): Euophrys confusa Kulcz., Bergut, Krk, 27.3., Damin. INV.NO.1740 (2 F): Euophrys confusa Kulcz., Šoići, 27.06.

Pseudeuophrys sp.

INV.NO.15 (1 JUV*): Heliophanus Kochii E. Simon, Borova draga, 07.07.1897. INV. NO.181 (1 JUV*): Euophrys sp., Malinska, 27.6.

Pseudeuophrys vafra (Blackwall, 1867)

INV.NO.192 (1 JUV*): Euophrys frontalis Walck., Bakar, Damin. INV.NO.193 (2 SUBM*, $\left.6 \mathrm{JUV}^{*}, 8 \mathrm{M}, 17 \mathrm{~F}\right)$ : Euophrys finitima E. Simon. INV.NO.196 (1 M, 1 F): Euophrys vafra, Borova draga, 8.7., Gery. INV.NO.247 (1): Euophrys vafra, Bag, 14.7.1899. INV.NO.261 (1 M): Euophrys vafra, Lukovo, 4.7.1899. INV.NO.297 (1 M, 2 F): Euophrys vafra, Sv. Juraj, 7.7.1911. INV.NO.1679 (2 M, 6 F): Euophrys finitima E. Sim., Bakar. INV.NO.1908 (2 F): Euophrys finitima E. Sim., Bag, 14.07.1899. INV.NO.2050 (1 M): Linyphia pusilla Sund. INV.NO.2268 (1 F): Euophrys sp., Malinska, 27.3. INV.NO.2291 (1 F): Euophrys imitata E. Sim., Kostrena, 22.4., Damin.

Pseudicius encarpatus (Walckenaer, 1802)

INV.NO.490 (1 M, 1 SUBM*, 2 JUV*, 2 F): Pseudicis encarpatus Walck., Bakar, Damin.

Saitis imitatus (Simon, 1868)

INV.NO.174 (3 JUV*): Euophrys imitata E. Simon, 4.5. INV.NO.190 (1 F): Euophrys imitata E. Simon, Malinska. INV.NO.191 (1 M): Euophrys imitata E. Simon, Kostrena, Sv. Lucija. INV.NO.246 (2 SUBM ${ }^{*}$ ): Euophrys imitata E. Simon, Kraljevica, 31.7.1897. INV. NO.334 (1 F): Euophrys rufibarbis E. Sim., Jablanac, 11.07., Damin. INV.NO.340 (1 JUV*, 3 SUBM): Euophrys imitata, Bakar, Krasica, Damin. INV.NO.341 (1 SUBM*): Euophrys imitata E. Sim., Voz, Krk, 7.6.1891., Damin. INV.NO.342 (5 F, 13 JUV*): Euophrys imitata E. Sim., Kostrena, 22.4., Damin. 
Salticidae gen. sp.

INV.NO.329 (2 JUV): Neon reticulatus Blackw., Bakar - uz more, 19.6.1893., Damin. INV.NO.1568 (1 JUV*): Aelurillus festivus C.L. Koch, Sn. Ujhely. INV.NO.2181 (1 JUV): Salticidae, Caska, 07.10.1955. INV.NO.2191 (1 F): Salticidae, Velo blato, o. Pag, 04.07.1956. INV.NO.2261 (1 JUV*): Drassodes lapidicola Wk., Gasperini.

Salticus cingulatus (Panzer, 1797)

INV.NO.53 (1 M): Epiblemum cingulatum Panz., Osijek, 1897.

\section{Salticus mutabilis Lucas, 1846}

INV.NO.68 (1 M): Epiblemum mutabile Lucas, Bakar. INV.NO.93 (2 M): Epiblemum mutabile Lucas, Ogulin, 19.04.1896. INV.NO.203 (1 JUV*, 1 SUBF*, 2 M, 10 F): Epiblemum mutabile Lucas, Kotor, Damin. INV.NO.204 (10 JUV*, 10 F, $32 \mathrm{M})$ : Epiblemum mutabile Lucas, Bakar, Kraljevica, Damin. INV.NO.1904 (2 F, 3 M): Epiblemum mutabile Lucas, Bakar. INV.NO.2428 (2 JUV*): Epiblemum mutabile Lucas, Bakar.

Salticus scenicus (Clerck, 1757)

INV.NO.45 (1 JUV*): Epiblemum scenicum Clerck., Topusko, 08.1891. INV.NO.205 (3 M, 5 F): Epiblemum scenicum Clerck., Zagreb, Damin. INV.NO.209 (1 M, 1 F): Epiblemum scenicum Clerck., Zemun, Damin.

Salticus sp.

INV.NO.2 (1 SUBF*): Salticus sp., Osijek, 1987., Langhoffer.

Salticus zebraneus (C. L. Koch, 1837)

INV.NO.54 (1 $\left.\mathrm{M}^{*}\right)$ : Epiblemum zebraneum C.L. Koch, Bakar. INV.NO.206 (1 M, 3 F): Epiblemum zebraneum C.L. Koch, Bakar, Damin. INV.NO.2058 (1 M, 1 F): Epiblemum zebraneum C.L. Koch. INV.NO.2270 (1 F): Epiblemum mutabile Lucas, Bakar, Kraljevica, Damin. INV.NO.2427 (1 F): Epiblemum mutabile Lucas, Bakar.

Sitticus atricapillus (Simon, 1882)

INV.NO.999 (1 M): Attus alpicola Kulcz., Risnjak, Damin.

Sitticus caricis (Westring, 1861)

INV.NO.1549 (1 F): Attus floricola C.L. Koch, Osijek, 1897., Langhoffer.

Sitticus damini (Chyzer, 1891)

INV.NO.300 (2 F): Attus Daminii Chyzer., Sv. Juraj, 17.08.1899., Damin. INV.NO.405 (6 F, 7 JUV*, 13 M): Attus Daminii Chyzer., Bakar, 05.06.1890., Damin. INV.NO.406 (3 JUV*, 3 F, 4 M): Attus Daminii Chyzer., Baška, Krk, 08.06.1987., Damin. INV.NO.407 (1 M, 1 SUBM$^{*}, 2$ JUV*, 4 F): Attus Daminii Chyzer., Kraljevica, Damin. INV.NO.408 (2 M): Attus Daminii Chyzer., Lukovo, 10.07.1899., Damin. INV.NO.1551 (1 F): Attus Daminii Chyzer., Žakalj, 17.09. INV.NO.1587 (1 F): Attus Daminii Chyzer., Borova draga, 08.07.

Sitticus dzieduszyckii (L. Koch, 1870)

INV.NO.996 (3 M, 3 F): Attus Dzieduszyckii L. Koch.

Sitticus floricola (C. L. Koch, 1837)

INV.NO.997 (1 F, 2 M): Attus floricola C.L. Koch. INV.NO.1557 (2 F): Attus floricola C.L. Koch. 
Sitticus pubescens (Fabricius, 1775)*

INV.NO.404 (1 JUV*, 2 F, 3 M): Attus poubescens F. INV.NO.1577 (1 F): Attus pubescens F., Osijek, 04.1899. INV.NO.1907 (2 JUV*): Attus pubescens F., Jablanac, 11.07.1899.

Sitticus rupicola (C. L. Koch, 1837)

INV.NO.2026 (2 F, 3 M): Attus rupicola C.L. Koch.

Sitticus sp.

INV.NO.996 (3 JUV): Attus Dzieduszyckii L. Koch.

Synageles dalmaticus (Keyserling, 1863)

INV.NO.70 (2 F): Synageles dalmaticus Keys., Prevez (Rasa), Damin. INV.NO.71 (1 F): Synageles dalmaticus Keys., Sv. Juraj, 07.07.1899., Damin. INV.NO.72 (1 F): Synageles dalmaticus Keys., Lukovo, 09.07., Damin. INV.NO.73 (2 F): Synageles dalmaticus Keys., Gasperini. INV.NO.1575 (1 F, 3 JUV $\left.^{*}\right)$ : Synageles dalmaticus Keys. INV.NO.2448 (1 SUBF*): Synageles hilarulus C.L. Koch.

\section{Scytodidae Blackwall, 1864}

Scytodes thoracica (Latreille, 1802)

INV.NO.147 (2 JUV*, 3 M, 12 F): Scytodes thoracica Latr., Bakar, Damin N. INV.NO.148 (1 F): Scytodes thoracica Latr., Arbanasi, Damin N. INV.NO.149 (2 F): Scytodes thoracica Latr., Gasperini. INV.NO.215 (2 F): Scytodes thoracica Latr., Zemun, Damin N. INV. NO.216 (1 M): Scytodes thoracica Latr., Novigrad, Damin N. INV.NO.289 (1 SUBF*, 1 F): Labulla thoracica Wid., Vrata (G. Kotar). INV.NO.1173 (2 JUV*): Scytodes thoracica Latr., Starigrad. INV.NO.1178 (1 JUV*): Scytodes thoracica Latr., Kostrena Sv. Barbara, 2.5.1890. INV.NO.1183 (1 JUV*, 1 M, 2 F): Scytodes thoracica Latr., Bakar. INV.NO.1187 (1 M, 1 F): Scytodes thoracica Latr., Bag, 19.7.1899. INV.NO.1192 (2 F): Scytodes thoracica Latr., Lukovo, 9.7.1899. INV.NO.2003 (1 SUBF*): Scytodes thoracica Latr., Jablanac, 11.07.1899. INV.NO.2010 (1 F): Scytodes thoracica Latr., Cavtat.

\section{Segestriidae Simon, 1893}

Segestria bavarica C. L. Koch, 1843

INV.NO.2263 (1 M): Segestria senoculata L. INV.NO.188 (1 JUV*): Segestria bavarica C.L. Koch, Bakar (janjac), Damin N. INV.NO.288 (2 JUV*): Segestria bavarica C.L .Koch, Bakar (janjac), Damin N. INV.NO.1407 (1 JUV*): Segestria bavarica C.L. Koch, Jablanac, 11.8. INV.NO.1420 (1 JUV*): Segestria bavarica C.L. Koch, Ogulin, 19.4.1896.

Segestria croatica Doleschall, $1852^{* * *}$

INV.NO.308 (1 JUV*): Segestria croatica Dolesch., Zadar, Damin N. INV.NO.1710 (2 $\left.\mathrm{JUV}^{*}\right)$ : Segestria croatica Dolesch., Bakar, Martinšćica.

Segestria florentina (Rossi, 1790)

INV.NO.159 (2 F, 27 JUV*): Segestria florentina Rossi. INV.NO.219 (2 JUV*, 2 F): Segestria fiorentina Rossi, Kotor, Damin N. INV.NO.465 (1 JUV*, 2 M, 2 F): Segestria florentina Rossi, Bakar, Damin N. INV.NO.466 (2 JUV*, 4 F): Segestria florentina Rossi. INV.NO.1944 (1 M): Segestria binoculata, Zagreb, Sofijin put, 06.07.1905. INV.NO.1948 (2 JUV*): Segestria florentina Rossi, Sv. Juraj, 07.07.1899. INV.NO.2077 (2 SUBF*): Segestria florentina Rossi, Caska, o. Pag, 22.06.1962. INV.NO.2078 (4 F): Segestria fiorentina Rossi, Caska, o. 
Pag, 25.06.1962. INV.NO.2098 (1 F): Segestria florentina Rossi, o. Pag, Kolansko blato, 27.06.1960. INV.NO.2102 (1 SUBF*): Segestria florentina Rossi, Caska, o. Pag, 12.06.1958. INV.NO.2152 (1 M, 2 F): Segestria florentina Rossi, Caska, o. Pag, 24.06.196o. INV.NO.2179 (1 M): Segestria florentina Rossi, Caska, o. Pag, 08.06.1956. INV.NO.2199 (1 F, 4 SUBF*): Segestria florentina Rossi, Unije, 25.06.1963. INV.NO.2208 (1 F): Segestria florentina Rossi, Biograd n/m, 04.08.1927., T. Šoljan.

Segestria senoculata (Linnaeus, 1758)***

INV.NO.175 (1 JUV*): Segestria senoculata L. INV.NO.978 (1 JUV*): Segestria senoculata L. Koch. INV.NO.1417 (1 JUV*): Segestria senoculata L. Koch, Skradin, 23.7.1897. INV. NO.1427 (1 JUV*): Segestria sp., Bakar. INV.NO.1936 (2 JUV*): Segestria senoculata Linn., Bakar, 02.09. INV.NO.1937 (1 JUV*): Segestria senoculata L. Koch, Bakar, 17.04.1903., Polić. INV.NO.1949 (1 JUV*): Segestria senoculata L. Koch, Bakar, 11.05.1903., Polić. INV. NO.2083 (1 JUV*): Segestria sp., Ćaska, o. Pag, 7.10.1955. INV.NO.2136 (3 SUBF*): Segestria sp., Unije, 29.06.1963., Magerle. INV.NO.2263 (1 JUV*): Segestria senoculata L.

\section{Sicariidae Keyserling, 1880}

Loxosceles rufescens (Dufour, 1820)

INV.NO.185 (2 JUV*): Loxosceles erythrocephalae C.L. Koch, Split, Damin N. INV. NO.186 (2 F): Loxosceles erythrocephalae C.L. Koch, Gasperini.

Loxosceles sp.

INV.NO.2288 (1 JUV*): Ozyptila horticola C.L. Koch, Gasperini.

\section{Sparassidae Bertkau, 1872}

Micrommata ligurina (C. L. Koch, 1845)

INV.NO.505 (1 F): Micrommata ligurina E. Sim., Gasperini. INV.NO.650 (1 SUBM*): Micrommata ligurina E. Sim., 28.3.

Micrommata sp.

INV.NO.2219 (1 SUBF): Chiracanthium sp., Zapeć (Plemenitaš) Gorski kotar, 1.7.1933. INV.NO.2273 (1 JUV): Epeira Sturmii Hahn., Sv. Filip i Jakov (Dalm.), Damin.

\section{Micrommata virescens (Clerck, 1757)}

INV.NO.291 (2 F, 4 JUV*): Micrommata virescens Clerck. INV.NO.292 (3 M): Micrommata virescens var. ornata Walck., Bakar. INV.NO.506 (1 JUV*, $1 \mathrm{M}, 1 \mathrm{~F})$ : Micrommata virescens Clerck., Žakalj (Rijeka), 17.10., Damin. INV.NO.507 (2 M, 3 JUV*, 5 F): Micrommata virescens Clerck. INV.NO.508 (1 SUBM*, 2 SUBF$\left.^{*}, 12 \mathrm{JUV}^{*}\right)$ : Micrommata virescens Clerck., Gasperini. INV.NO.1959 (1 F): Micrommata virescens Clerck., Plitvice, 26.08.1902. INV.NO.1965 (1 F): Micrommata virescens Clerck., Podsused, 08.05.1915. INV.NO.1968 (1 M): Micrommata virescens Clerck., Bakar, 11.05.1903., Polić.

Micrommata virescens ornata (Walckenaer, 1802)

INV.NO.292 (3 SUBM ${ }^{*}, 8$ JUV$^{*}$ ): Micrommata virescens var. ornata Walck., Bakar. INV. NO.509 (11 JUV*): Micrommata virescens var. ornata Walck., Gasperini. INV.NO.1694 (10 $\mathrm{JUV}^{*}$ ): Micrommata virescens var. ornata Walck., Bakar, Damin. INV.NO.1958 (2 JUV*): Micrommata virescens var. ornata Walck., Bakar, 09.01.1895., Damin. 
Micrommata virescens (Clerck, 1757)

INV.NO.692 (1 JUV*): Micrommata virescens Clerck., Petrovaradin-Irig, 12.8.1901. INV. NO.696 (1 JUV*): Micrommata virescens Clerck., Bribir-Novi, 15.4.1897. INV.NO.732 (1 $J_{U V}^{*}$ ): Micrommata virescens Clerck., Rijeka, 1.10.1901. INV.NO.743 (1 JUV*): Micrommata virescens Clerck., Kraljičin zdenac, 18.9.1901. INV.NO.753 (1 JUV*): Micrommata virescens Clerck., Bribir-Novi, 15.4.1897. INV.NO.774 (1 JUV*): Micrommata virescens Clerck. INV.NO.1685 (2 JUV*): Micrommata virescens Clerck., Kraljičin zdenac, 14.6.1897., Dr. Langhoffer. INV.NO.1961 (1 JUV*): Micrommata virescens Clerck., Ivanšćica, 26.07.1915. INV.NO.1962 (1 JUV*): Micrommata virescens Clerck., Mošćenice, 01.09.1911. INV. NO.1964 (1 JUV*): Micrommata virescens Clerck., Bakar, 29.03.1903., Polić. INV.NO.1966 (1 JUV*): Micrommata virescens Clerck., Bakar, 03.10.1903. INV.NO.1967 (1 SUBM ${ }^{*}, 3$ $\left.\mathrm{JUV}^{*}\right)$ : Micrommata virescens Clerck., Gasperini. INV.NO.1971 (1 JUV*): Micrommata virescens Clerck., Mrkvišće, 28.07.1912. INV.NO.1977 (1 JUV*): Micrommata virescens Clerck., Sljeme, 10.09.1905., Siegl.

Olios argelasius (Walckenaer, 1806)

INV.NO.302 (1 M, 2 JUV*$^{*}, 2$ SUBM$\left.^{*}\right)$ : Olios spongitarsis Dufour. INV.NO.617 (1SSP*): Olios spongitarsis Dufour. INV.NO.640 (2SSP*): Olios spongitarsis Dufour., Bakar. INV. NO.691 (1 JUV*): Olios spongitarsis Dufour., Starigrad. INV.NO.954 (2 JUV*): Olios spongitarsis Dufour., Kotor, 03.08.1894., Damin. INV.NO.955 (1): Olios spongitarsis Dufour., Starigrad, Damin.

Olios sp.

INV.NO.860 (2 JUV): Philodromus lividus Sim., Brusje, Damin. INV.NO.2369 (2 JUV): Philodromus lividus Sim.,

\section{Tetragnathidae Menge, 1866}

Meta menardi (Latreille, 1804)

INV.NO.902 (1 M, 1 SUBM* 2 F): Meta Menardi Latr., Pazarište. INV.NO.1144 (2 JUV*): Meta Menardi Latr., 12.08.1902., Siničić. INV.NO.1625 (1 M): Meta segmentata Clerck., Martinka, 20.07.1915. INV.NO.1628 (1 M, 1 F): Meta segmentata Clerck., Ozalj. špilja, 17.06.1917. INV.NO.1826 (2 F): Meta segmentata Clerck., špilja Golubnjača, 05.03.1913., Koch. INV.NO.2067 (1 SUBM*, 2 F, 4 JUV*): Meta Menardi Latr., Zečev varoš-Pećina, 20.07.1913. INV.NO.2201\#: Meta Menardi Latr., špilja Zapeć, Gorski kotar, 12.08.1908., Dr. Babić. INV.NO.2202 (1 M): Meta Menardi Latr., Zapeć (Plemenitaš) Gorski kotar, 07.1933. INV.NO.2215 (1 cocoon*): Meta Menardi Latr., Vrana peć., 21.08.1916. INV.NO.2221\#: Meta menardi. INV.NO.2232 (1 cocoon*): Meta Menardi Latr., Jablanac, 21.07.1916.

\section{Meta sp.}

INV.NO.1608 (1 SUBF): Zilla litterata Olv., 13.9.1916.

Metellina mengei (Blackwall, 1870)

INV.NO.909 (2 M): Meta segmentata var. Mengei Thor., Zagreb. INV.NO.910 (2 F): Meta segmentata var. Mengei Thor., Bakar. INV.NO.1116 (1 M, 3 F): Meta segmentata var. Mengei Thor., Kraljičin zdenac, 14.04.1897. INV.NO.1117 (1 F): Meta segmentata var. Mengei Thor., Zagreb-Sofijin put, 14.06.1898. INV.NO.1130 (1 F): Meta segmentata var. Mengei Thor., Bribir-Novi, 15.04.1892. INV.NO.1159 (2 F): Meta segmentata var. Mengei Thor. INV.NO.1166 (1 M): Meta segmentata var. Mengei Thor., Grižane, 14.04. INV. NO.2375 (2 M): Meta segmentata Clerck., Koloszvar. 
Metellina merianae (Scopoli, 1763)

INV.NO.903 (1 M): Meta Merianae Scop., Pećine (vrelo) Dalm. INV.NO.904 (1 F): Meta Merianae Scop., Pećine (Vrana). INV.NO.905 (2 M, 2 SUBM*, 3 JUV*, 10 F): Meta Merianae Scop., Bakars. Draga. INV.NO.908 (2 F): Meta segmentata Clerck., Mrzla vodica. INV. NO.917 (1 JUV*, 1 M, 6 F): Meta Merianae Scop., Risnjak, Damin. INV.NO.1118 (1 F): Meta Merianae Scop., Bakar (kanal), 23.07.1902. INV.NO.1132 (1 JUV*, 1 F): Meta Merianae Scop., Zagreb, 07.09.1902. INV.NO.1139 (1 M, 6 JUV*): Meta Merianae Scop., BribirNovi, 15.04.1897. INV.NO.1200 (1 SUBM*): Meta Merianae Scop., Bribir-Novi, 15.04.1892. INV.NO.1569 (2 JUV*): Meta Merianae Scop., Bakar, Damin. INV.NO.1618 (2 F): Meta segmentata Clerck., Ozalj. špilja, 17.06.1917.

\section{Metellina segmentata (Clerck, 1757)}

INV.NO.906 (1 SUBM*, 3 F): Meta segmentata Clerck., Kraljičin zdenac. INV.NO.907 (3 M, 9 F): Meta segmentata Clerck., Koloszvar. INV.NO.1131 (1 F): Meta segmentata var. Mengei Thor., Zagreb, 1898. INV.NO.1137 (5 F): Meta segmentata Clerck., Osijek, 1898. INV. NO.1138 (1 SUBM ${ }^{*}$, 2 JUV*): Meta segmentata Clerck., Jasikovac, 18.08.1902. INV.NO.1143 $\left(2 \mathrm{JUV}^{*}\right)$ : Meta segmentata Clerck., Plitvice, 18.08.1898. INV.NO.1150 (1 M, 3 JUV*, 4 F): Meta segmentata Clerck., Zagreb, 1897., Langhoffer. INV.NO.1165 (2 F): Meta segmentata var. Mengei Thor., Rijeka, 23.09.1894. INV.NO.1625 (1 JUV*, 1 SUBM*): Meta segmentata Clerck., Martinka, 20.07.1915. INV.NO.1631 (1 JUV*): Meta segmentata Clerck., Vrana peć., 23.08.1916. INV.NO.1649 (1 F): Zilla litterata Olv., Delnice, 06.06.1912., Langhoffer. INV.NO.1655 (1 F): Zilla sp., Mošćenice, 1.9.1911. INV.NO.1658 (1 M): Meta segmentata Clerck., Zagreb, 29.11.1916. INV.NO.1672 (1 F): Zilla Stroemii Thor., Samobor, 08.09.1911. INV.NO.1852 (2 SUBM ${ }^{*}, 3$ JUV$^{*}$ ): Meta segmentata Clerck., Vrana, Peć, 21.08.1916. INV. NO.1989 (1 M): Linyphia montana Clerck., Zagreb-Tuškanac, 12.10.1901. INV.NO.2376 (3 SUBM$^{*}, 8 \mathrm{JUV}^{*}$ ): Meta segmentata Clerck., Koloszvar. INV.NO.2400 (5 F): Epeira Sturmii Hahn., Osijek, 1897., Dr. Langhoffer. INV.NO.2406 (3 JUV*): Meta segmentata Clerck., Ozalj. špilja, 17.06.1917. INV.NO.2451 (1SSP*): Meta segmentata Clerck., Kolansko blato, 04.10.1957.

Pachygnatha clercki Sundevall, 1823

INV.NO.89 (1 F, 3 M): Pachygnatha Clerckii Sund., Osijek, Damin.

\section{Pachygnatha degeeri Sundevall, 1830}

INV.NO.86 (4 F): Pachygnatha Degeerii Sund., Bakar, Damin. INV.NO.87 (1 SUBM*, 3 M, 8 F): Pachygnatha Degeerii Sund., Osijek, Damin. INV.NO.88 (1 M, 1 F): Pachygnatha Degeerii Sund., Mrzla vodica, Damin. INV.NO.1115 (1 F): Pachygnatha Degeerii Sund., Osijek, 4.1899. INV.NO.1129 (1 F): Pachygnatha Degeerii Sund., 1901., Trgovčević. INV. NO.1136 (1 F, 3 M): Pachygnatha Degeerii Sund., Kecskemet, 03.1890., Salzteich. INV. NO.1151 (1 F): Pachygnatha Degeerii Sund., Osijek, 1907. INV.NO.1164 (1 SUBM*, 1 F): Pachygnatha Degeerii Sund., Stara Pazova, 10.8.1898. INV.NO.2045 (1 M, 1 SUBM*, 4 F): Pachygnatha Degeerii Sund. INV.NO.2254 (1 M): Pachygnatha Clerckii Sund., Osijek, Damin.

Tetragnatha extensa (Linnaeus, 1758)

INV.NO.112 (8 SUBM*, 12 SUBF*, 93 JUV*): Tetragnatha extensa Linn., Osijek, Damin. INV.NO.113 (4 F): Tetragnatha extensa Linn., Draga, Damin. INV.NO.114 (2 M): Tetragnatha extensa Linn., Vrana (Dalm.), Damin. INV.NO.115 (1 F): Tetragnatha extensa Linn., Novigrad (Dalm.), Damin. INV.NO.700 (2 M, 2 F): Tetragnatha Solandrii Scop., Božjakovina, Langhoffer. INV.NO.1114 (1 JUV, 1 F): Tetragnatha extensa Linn., Osijek, 7.1901., Rössler. INV.NO.1135 (1 F): Tetragnatha extensa Linn., Stara Pazova, 10.8.1898. 
INV.NO.1142 (1 M, 1 F): Tetragnatha extensa Linn., Osijek, 1897., Langhoffer. INV.NO.1149 (1 SUBM*): Tetragnatha extensa Linn., Kupinovo, 19.7.1902., Rössler. INV.NO.1156 (1 M, 3 F): Tetragnatha extensa Linn., Križevci, 4.9.1897., Langhoffer. INV.NO.1162 (1 M, 1 F): Tetragnatha extensa Linn., Ogulin. INV.NO.1617 (1 M): Tetragnatha extensa Linn., Božjakovina, 30.9.1910. INV.NO.1652 (1 JUV*): Tetragnatha extensa Linn., Crna mlaka, 30.6.1907., Langhoffer. INV.NO.1662 (1 M): Tetragnatha extensa Linn., Obeda, 24.5.1912. INV.NO.1665 (1 JUV*): Tetragnatha extensa Linn., Boljevci, 21.5.1912. INV.NO.1668 (2 SUBF*, 3 F): Tetragnatha extensa Linn. INV.NO.1669 (3 M, 3 F): Tetragnatha sp., Babina greda, 17.7.1918. INV.NO.1671 (1 SUBM*, 4 F): Tetragnatha extensa Linn., Crna mlaka, 28.8.1912. INV.NO.1827 (1 JUV*): Tetragnatha extensa Linn., Jankovac, 04.06.1902. INV. NO.1836 (1 JUV*, 4 F): Tetragnatha extensa Linn., Oni?, 27.08.1903. INV.NO.1850 (1 M, 4 JUV* $^{*} 4$ SUBM$^{*}$ ): Tetragnatha extensa Linn., Martinšćica, Damin. INV.NO.1859 (2 M, 2 F): Tetragnatha extensa Linn., Klanjec, 1904., Broz. INV.NO.1860 (1 M): Tetragnatha extensa Linn., Vrpolje, 15.07.1912. INV.NO.2416 (2 M): Pisaura mirabilis CI., Vrbica. INV.NO.700 (1 JUV*): Tetragnatha Solandrii Scop., Božjakovina. INV.NO.1140 (1 SUBM*): Tetragnatha Solandrii Scop., Bribir-Novi, 15.4.1897. INV.NO.2327 (2 SUBM*, 3 JUV*): Tetragnatha Solandrii Scop., izvor Rječine, 25.05.1890., Damin.

Tetragnatha montana Simon, $1874^{*}$

INV.NO.699 (1 F): Tetragnatha Solandrii Scop., Vrbica, Damin. INV.NO.701 (4 F): Tetragnatha Solandrii Scop., Ogulin. INV.NO.702 (1 M, 1 F): Tetragnatha Solandrii Scop., izvor Rječine, 25.05.1890., Damin. INV.NO.1127 (1 F): Tetragnatha Solandrii Scop., Osijek, 1898. INV.NO.1146 (1 M): Tetragnatha Solandrii Scop., 1901., Trgovčević. INV.NO.1148 (1 F): Tetragnatha Solandrii Scop., Zagreb, 1898. INV.NO.1624 (1 F): Tetragnatha extensa Linn., Remete, 28.5.1905. INV.NO.1827 (1 F): Tetragnatha extensa Linn., Jankovac, 04.06.1902. INV.NO.1838 (1 F): Tetragnatha extensa Linn., Lapinska rijeka kod Nikolićeva. INV.NO.2256 (1 F): Tetragnatha extensa Linn., Osijek, Damin.

Tetragnatha nigrita Lendl, $1886^{*}$

INV.NO.1128 (1 F): Tetragnatha nigrita Lendl. INV.NO.1133 (1 M, 1 F): Tetragnatha sp., Osijek, 1897. INV.NO.1134 (1 F): Tetragnatha nigrita Lendl., Ogulin, 4.6.1898. INV.NO.1849 (1 F, 2 M): Tetragnatha extensa Linn., Soči, 12.08.1903. INV.NO.116 (1 JUV*): Tetragnatha nigrita Lendl., Bakar, Damin.

Tetragnatha obtusa C.L. Koch, 1837

INV.NO.110 (1 M): Tetragnatha obtusa C.L. Koch, Vrana, Damin. INV.NO.111 (11 JUV*): Tetragnatha obtusa C.L. Koch, Mrzla vodica, Damin. INV.NO.698 (2 F): Tetragnatha pinicola L. Koch, Bitoraj, 26.06.1892., Damin. INV.NO.1112 (1 JUV*, 1 SUBM $^{*}, 1$ SUBF*): Tetragnatha obtusa C.L. Koch, Osijek, 1897., Langhoffer. INV.NO.1141 (2 JUV*): Tetragnatha obtusa C.L. Koch, Osijek, 1897., Langhoffer. INV.NO.1147 (1 F): Tetragnatha obtusa C.L. Koch, Plitvice, 27.8.1902. INV.NO.1154 (6 JUV*): Tetragnatha obtusa C.L. Koch, Krk. INV.NO.1155 (2 JUV*): Tetragnatha obtusa C.L. Koch, Bitoraj-podnožje, 26.4.1898. INV.NO.1638 (1 F): Tetragnatha obtusa C.L. Koch, Fužine, Damin. INV.NO.2063 (3 F): Tetragnatha pinicola L. Koch. INV.NO.2326 (1 F): Tetragnatha Solandrii Scop., izvor Rječine, 25.05.1890., Damin. INV.NO.2384 (1 F): Tetragnatha Solandrii Scop., Osijek, 1898.

Tetragnatha pinicola L. Koch, 1870

INV.NO.698 (3 JUV*): Tetragnatha pinicola L. Koch, Bitoraj, 26.06.1892., Damin. INV. NO.1161 (1 M): Tetragnatha pinicola L. Koch. INV.NO.2063 (1 JUV*): Tetragnatha pinicola L. Koch. 
Tetragnatha sp.

INV.NO.1145 (14 JUV): Tetragnatha sp., Topusko, 8.1891. INV.NO.1669 (3 JUV, 3 SUBM): Tetragnatha sp., Babina greda, 17.7.1918. INV.NO.2233 (2 F): Tetragnatha sp. INV.NO.2439 (1 JUV): Pholcus opilionides Schr., Buk Krke.

Tetragnathidae gen. sp.

INV.NO.1191 (1 JUV): Uloborus Walckenaeri Latr., Skradin, 23.03.1894.

\section{Theridiidae Sundevall, 1833}

Anelosimus pulchellus (Walckenaer, 1802)

INV.NO.210 (1 F): Theridium pulchellum Walck., Crikvenica, 12.8.1893., Damin N. INV. NO.801 (1 JUV*, 1 M, 2 F): Theridium pulchellum Walck., Porto Palazzo, 15.5.1890., Damin N. INV.NO.867 (2 F): Theridium riparium Blackw., Osijek, Damin N.

Anelosimus sp.

INV.NO.1980 (1 SUBM): Walckenaera antica Wid., Lunte?

Argyrodes argyrodes (Walckenaer, 1841)

INV.NO.48 (1 F): Argyrodes epeira Sim., Brusje, o. Hvar, Damin N.

Asagena phalerata (Panzer, 1801)

INV.NO.235 (1 M, 2 F, 3 SUBM*, 8 JUV*): Asagena phalerata Panz., Bakar. INV.NO.236 (2 SUBM ${ }^{*}$ ): Asagena phalerata Panz., Senj. INV.NO.237 (3 JUV*, 7 SUBM*): Asagena phalerata Panz. INV.NO.1993 (1 SUBM*): Asagena phalerata Panz., Fužine, 12.08., Damin.

Crustulina guttata (Wider, 1834)

INV.NO.1260 (1 M): Crustulina guttata Wid., Kraljevica, 31.8.1899.

Crustulina scabripes Simon, 1881

INV.NO.750 (1 SUBM*, 2 F): Crustulina scabripes E. Sim., Buk Krke, Damin N. INV. NO.751 (1 F): Crustulina scabripes E. Sim., Povile, 9.7., Damin N. INV.NO.752 (1 M, 2 F): Crustulina scabripes E. Sim., Lukovo, Damin N. INV.NO.1268 (1 F): Crustulina scabripes E. Sim., Novi, 19.7. INV.NO.2274 (1 F): Asagena phalerata Panz., Bakar.

Crustulina sp.

INV.NO.1279 (1 SUBF): Crustulina sp., Kraljevica, 8.7.

Dipoena croatica (Chyzer, 1894)

INV.NO.784 (1 M, 1 SUBF*): Dipoena croatica Chyz., Omišalj, Damin N. INV.NO.785 (2 $J_{U V}^{*}$ ): Dipoena croatica Chyz., Bakar, Damin N. INV.NO.786 (1 JUV*): Dipoena croatica Chyz., Omišalj, Damin N.

Dipoena melanogaster (C. L. Koch, 1837)

INV.NO.79 (8 F): Dipoena melanogaster C.L. Koch, Bakar, 21.05.1890., Damin. INV. NO.80 (1SSP*): Dipoena melanogaster C.L. Koch, Zagreb, Damin. INV.NO.1238 (1 F): Nesticus cellulanus Clerck., 12.8.1902., Sinčić. INV.NO.1249 (1SSP*): Dipoena melanogaster C.L. Koch, Krk. INV.NO.1250 (7 JUV*): Dipoena melanogaster C.L. Koch, 20.05.1891.

Dipoena sp.

INV.NO.2272 (1 JUV): Scytodes thoracica Latr., Zemun, Damin N. INV.NO.2394 (1SSP): Clubiona trivialis C.L. Koch, Bitoraj, podnožje, 26.6.1892. 
Enoplognatha afrodite Hippa \& Oksala, 1983

INV.NO.493 (1 F): Theridium lineatum Clerck., Senj, Damin N. INV.NO.2304 (2 F): Theridium lineatum Clerck., Punat (Krk), Damin N. INV.NO.2342 (2 M, 19 F): Theridium lineatum Clerck., Bakar, 5.6.1898., Damin N.

Enoplognatha latimana Hippa \& Oksala, 1982

INV.NO.1991 (1 F): Theridium lineatum Clerck., Bešinovo, 27.07.1917.

Enoplognatha mandibularis (Lucas, 1846)

INV.NO.65 (2 F): Enoplognatha mandibularis Luc., Gasperini. INV.NO.66 (3 F): Enoplognatha mandibularis Luc., Split, Damin. INV.NO.2262 (3 F): Filistata testacea.

Enoplognatha ovata (Clerck, 1757)

INV.NO.493 (1 JUV*): Theridium lineatum Clerck., Senj, Damin N. INV.NO.494 (1 F, 2 M): Theridium lineatum Clerck., Punat (Krk), Damin N. INV.NO.690 (2 JUV*): Theridium lineatum Clerck., Glavotok (Krk), 12.8.1893., Damin N. INV.NO.748 (2 F): Theridium lineatum Clerck., Bakar, 5.6.1898., Damin N. INV.NO.1205 (1 SUBM* ${ }^{*} 2$ F): Theridium lineatum Clerck., Križevci, 4.9.1897. INV.NO.1210 (1 M): Theridium lineatum Clerck., Jasikovac, 16.8.1902. INV.NO.1211 (6 JUV*): Theridium lineatum Clerck., Krk. INV.NO.1245 (1 F): Theridium lineatum Clerck., Bregi, 13.7.1902. INV.NO.1246 (1 SUBM*): Theridium lineatum Clerck., Kraljičin zdenac, 14.6.1897., Langhoffer. INV.NO.1252 (1 M): Theridium lineatum Clerck., Sofijin put, 19.6.1898. INV.NO.1693 (1 F): Theridium lineatum Clerck., Arbanasi. INV.NO.2305 (1 SUBM ${ }^{*}$ ): Theridium lineatum Clerck., Punat (Krk), Damin N. INV.NO.2342 (2 M): Theridium lineatum Clerck., Bakar, 5.6.1898., Damin N. INV.NO.2343 (3 SUBM*, 14 JUV $^{*}$ ): Theridium lineatum Clerck., Bakar, 5.6.1898., Damin N.

Episinus cavernicola (Kulczyński, 1897)

INV.NO.550 (1 JUV*, 2 SUBM*): Plocamis cavernicola Kulcz., Bakar (u vrhu), 12.05.1890., Damin N. INV.NO.1543 (1 F): Plocamis cavernicola Kulcz., INV.NO.1881 (1 F): Micaria nivosa L. Koch, Jelenje, 29.08.1913.

Episinus maculipes Cavanna, 1876

INV.NO.834 (1 M, 1 F): Episinus truncatus Latr., Košljun, 18.7., Damin N.

Episinus truncatus Latreille, 1809

INV.NO.802 (1 JUV*, 1 M, 1 SUBM* ${ }^{*} 2$ F): Theridium pulchellum Walck., Porto Palazzo, 5.4.1890., Damin N. INV.NO.832 (1 M): Episinus lugubris E. Sim., 24.6., Damin N. INV. NO.833 (2 JUV*, 2 F, 8 M): Episinus lugubris E. Sim., Bakarac, 24.7., Damin N.

Euryopis episinoides (Walckenaer, 1847)

INV.NO.558 (1 F): Euryops acuminata Luc., Bakar, 4.8., Damin N. INV.NO.675 (1 F): Euryops acuminata Luc., Budva, Damin N. INV.NO.790 (1 M, $\left.2 \mathrm{JUV}^{*}\right)$ : Euryops acuminata Luc., Split, 27.7.1894., Damin N. INV.NO.1227 (1 F): Euryops acuminata Luc., Budva.

Euryopis margaritata (L. Koch, 1867)

INV.NO.559 (1 M*, 2 F): Euryops margaritata L. Koch, Bakar (ispod Kalvarije), 5.6., Damin N. INV.NO.560 (1 JUV*, 1 SUBM*): Euryops margaritata L. Koch, Kostrena, 22.4., Damin N. 
Heterotheridion nigrovariegatum (Simon, 1873)

INV.NO.761 (2 JUV*, 2 F, $3 \mathrm{M}$ ): Theridium nigrovariegatum Sim., Bakar (nad kupalištem), 12.6.1890., Damin N. INV.NO.2344 (2 M): Theridium lineatum Clerck., Bakar, 5.6.1898., Damin N. INV.NO.2460 (2 SUBM*, 3 M, 5 JUV*): Theridium nigrovariegatum Sim., Sv. Kuzma, 03.06.1890., Damin N.

Kochiura aulica (C. L. Koch, 1838)

INV.NO.182 (1 M): Theridium aulicum C.L. Koch, Omišalj, Damin N. INV.NO.211 (1 M, 3 SUBM$^{*}, 5$ F): Theridium aulicum C.L. Koch, Crikvenica, 12.8.1899., Damin N. INV. NO.212 (1 JUV*): Theridium aulicum C.L. Koch, Stari grad, Damin N. INV.NO.803 (2 F): Theridium aulicum C.L. Koch, Cavtat, Damin N. INV.NO.864 (1 F): Theridium aulicum C.L. Koch, Porto Palazzo, Damin N. INV.NO.865 (2 F): Theridium aulicum C.L. Koch, Vis, 7.8., Damin N. INV.NO.1215 (1 M): Theridium aulicum C.L. Koch. INV.NO.1228 (2 F): Theridium aulicum C.L. Koch. INV.NO.1979 (1 SUBM*, 1 F): Theridium aulicum C.L. Koch, Cavtat, 12.1898. INV.NO.2458 (1 F): Theridium aulicum C.L. Koch, Vis, Damin N. INV.NO.2459 (1 M, 1 F): Theridium aulicum C.L. Koch.

Lasaeola convexa (Blackwall, 1870)

INV.NO.799 (1 F, 2 JUV): Dipoena convexa Blackw., Bakar. INV.NO.1202 (1SSP*): Dipoena convexa Blackw., Križišće, 06.08.1893. INV.NO.1208 (1 F): Dipoena convexa Blackw., Bakar. INV.NO.1982 (1 M): Dipoena convexa Blackw., Kotor, 03.08.1899. INV. NO.2373 (1 M): Thanatus vulgaris E. Sim., ?, Bakar.

Lasaeola tristis (Hahn, 1833)

INV.NO.2053 (3 M, 13 F): Epeira umbratica Clerck., Šestine.

Latrodectus tredecimguttatus (Rossi, 1790)

INV.NO.641 (2 JUV*): Lathrodectus tredecim-guttatus Rossi, Krk (Voz), 6.7.1891., Damin N. INV.NO.642 (1 SUBM*): Lathrodectus tredecim-guttatus Rossi, Lukovo, 9.7.1899., Damin N. INV.NO.643 (1 JUV*): Lathrodectus tredecim-guttatus Rossi, Damin N. INV. NO.644 (3 F): Lathrodectus tredecim-guttatus Rossi, Sv. Filip i Jakov (Dalm.), Damin N. INV.NO.645 (2 F): Lathrodectus tredecim-guttatus Rossi, Sv. Filip i Jakov (Dalm.), Damin N. INV.NO.646 (1 F, 229 JUV*): Lathrodectus tredecim-guttatus Rossi, Korenić, 29.3.1893., Damin N. INV.NO.647 (2 F): Lathrodectus tredecim-guttatus Rossi, Gasperini. INV.NO.648 (1 F): Lathrodectus tredecim-guttatus Rossi, Bakar. INV.NO.687\#: Lathrodectus tredecimguttatus Rossi, Bakar. INV.NO.688 (2 F): Lathrodectus tredecim-guttatus Rossi, Sv. Filip i Jakov (Dalm.). INV.NO.1253 (6 JUV*): Lathrodectus tredecim-guttatus Rossi, Voz-Vitezov. Špilja, 7.7.1891. INV.NO.1981 (4 M): Lathrodectus tredecim-guttatus Rossi, Sv. Filip i Jakov (Dalm.), 1897., Damin N. INV.NO.1985 (3 F): Lathrodectus tredecim-guttatus Rossi, Sv. Filip i Jakov (Dalm.). INV.NO.1995 (1 F, 2 M): Lathrodectus tredecim-guttatus Rossi, Bakar, Damin N. INV.NO.2071 (1 F): Lathrodectus tredecim-guttatus Rossi, Kolansko blato, o. Pag, 28.09.1957.

Neottiura herbigrada (Simon, 1873)

INV.NO.806 (1 M): Theridium herbigradum E. Sim., Omišalj, Damin N. INV.NO.807 (1 SUBM $^{*}$ ): Theridium herbigradum E. Sim., Bakar, Damin N.

Parasteatoda lunata (Clerck, 1757)

INV.NO.143 (2 M, 2 F): Theridium formosum Clerck., Bakar, 16.08., Damin N. INV. NO.189 (2 JUV*, 4 M, 35 F): Theridium formosum Clerck., Ogulin, Damin N. INV. 
NO.208 (4 M, 4 F): Theridium formosum Clerck., Fužine, 14.5., Damin N. INV.NO.1229 (1 F): Theridium formosum Clerck. INV.NO.1232 (1 JUV*): Theridium formosum Clerck., 1901., Trgovčević. INV.NO.1235 (1 SUBM*): Theridium formosum Clerck., Topusko. INV. NO.1240 (1 JUV*): Theridium formosum Clerck. INV.NO.1986 (1 F): Theridium umbraticum L. Koch, Tuškanac, 14.05.1907. INV.NO.1994 (1 F): Theridium formosum Clerck., Klanjec, 14.05.1907.

Parasteatoda simulans (Thorell, 1875)*

INV.NO.1225 (1 F): Theridium simulans, Osijek, 12.1898.

Parasteatoda sp.

INV.NO.868 (3 SUBM, 3 SUBF): Theridium aulicum C.L. Koch. INV.NO.1233 (4 JUV): Episinus sp. INV.NO.1234 (2 JUV): Dipoena sp., Krk.

Parasteatoda tepidariorum (C. L. Koch, 1841)

INV.NO.631 (2 M, 3 SUBM$^{*}, 4$ JUV$^{*}, 6$ F): Theridium tepidariorum C.L. Koch, Zemun, 1899., Damin N. INV.NO.868 (2 M, 22 F): Theridium aulicum C.L. Koch. INV.NO.1263 (1 F): Theridium tepidariorum C.L. Koch, Topusko, 8.1891. INV.NO.1271 (1 F): Theridium tepidariorum C.L. Koch, Topusko. INV.NO.1276 (1 M): Theridium tepidariorum C.L. Koch, Topusko, 8.1891.

Phycosoma inornatum (O. P.-Cambridge, 1861)

INV.NO.786 (1 F): Dipoena croatica Chyz., Omišalj, Damin N. INV.NO.798 (1 M, 1 F): Dipoena inornata O.P. Cambr., Bakar.

Phylloneta impressa (L. Koch, 1881)

INV.NO.760 (1 SUBF*, 1 F, 2 JUV*): Theridium impressum L. Koch, Bakar, Senj, Damin N. INV.NO.1216 (1 F): Theridium impressum L. Koch, Plitvice, 27.8.1902. INV.NO.1996 (1 M, 5 F): Theridium impressum L. Koch, Vrata, 26.06.1892.

Phylloneta sisyphia (Clerck, 1757)*

INV.NO.749 (1 M, 1 F, 6 JUV*): Theridium sisyphium Clerck. Fužine, Damin N. INV. NO.1206 (1 F): Theridium sisyphium Clerck., Kraljičin zdenac, 14.6.1897., Langhoffer. INV.NO.1696 (1 F): Theridium sisyphium Clerck., Mrzla vodica. INV.NO.2366 (2 F, 4 M): Linyphia peltata Wid., Zagreb. gora, Damin.

Platnickina sp.

INV.NO.2024 (1 JUV*): Theridium tinctum Walck., Topusko.

Platnickina tincta (Walckenaer, 1802)

INV.NO.532 (1 F, 2 M): Theridium pictum Walck., Božjakovina, Damin N. INV.NO.633 (1 F): Theridium tinctum Walck., Božjakovina, Damin N. INV.NO.1281 (2 F): Theridium tinctum Walck., Vrata, 26.6.1892. INV.NO.630 (1 JUV*): Theridium tinctum Walck., Bakar, 18.4.1891., Damin N. INV.NO.1704 (1 SUBM*): Theridium tinctum Walck., Mrzla vodica.

Rhomphaea nasica (Simon, 1873)

INV.NO.117 (1 M): Ariamnes nasicus Sim., Lokrum, Damin N.

Robertus arundineti (O. P.-Cambridge, 1871)*

INV.NO.1570 (1 F): Pachygnatha Clerckii Sund., Kot Sv. Ivana - Krk. INV.NO.1585 (2 M, 3 F): Pachygnatha Clerckii Sund., Kecskemet, 1890. 
Simitidion simile (C. L. Koch, 1836)

INV.NO.56 (1 JUV*): Theridium simile C.L. Koch, Fužine, 22.08.1884., Damin N. INV. NO.141 (1 M, 12 F): Theridium simile C.L. Koch, Bakar,Primorje, Damin N. INV.NO.142 (1 F, 19 JUV $^{*}, 25$ SUBM$\left.^{*}\right)$ : Theridium simile C.L. Koch, Dalmacija, Damin N. INV.NO.1230 (1 SUBM$^{*}$, 2 JUV*): Theridium simile C.L. Koch, Mali Grad. INV.NO.1231 (3 F): Theridium simile C.L. Koch, Bribir-Novi, 15.4.1893. INV.NO.1236 (1 M): Theridium simile C.L. Koch, Bakar. INV.NO.2322 (1 F): Heriaeus Savignyi E. Sim., Kotor, Damin.

Steatoda bipunctata (Linnaeus, 1758)

INV.NO.987 (1 JUV*): Steatoda bipunctata L. Koch, Mrzla vodica, Damin N. INV. NO.988 (7 JUV*): Steatoda bipunctata L., Zemun, 1899., Damin N. INV.NO.1254 (1 $\mathrm{SUBM}^{*}$ ): Steatoda bipunctata L. Koch, Topusko, 8.1891. INV.NO.1269 (1 SUBM*, 2 JUV*): Steatoda bipunctata L. Koch, Topusko. INV.NO.1289 (1 SUBM ${ }^{*}$ : Steatoda bipunctata L. Koch, 1901. INV.NO.1291 (1 M): Steatoda bipunctata L. Koch, Osijek, 4.1899. INV.NO.1745 (1 M, 1 SUBM* 1 F, 2 JUV*): Steatoda bipunctata L. Koch, Risnjak, Damin N. INV.NO.1990 (4 JUV*): Steatoda bipunctata L. Koch, Topusko, 08.1891. INV.NO.1998 (1 F): Steatoda bipunctata L. Koch, Ogulin, 06.02. INV.NO.2023 (1 F): Steatoda bipunctata L. Koch, Ogulin, 19.04.1892. INV.NO.2357 (2 F): Teutana castanea Clerck., Osijek, Damin N.

Steatoda castanea (Clerck, 1757)

INV.NO.796 (1 SUBF*, 2 M, 3 SUBM*, 7 JUV*, 8 F): Teutana castanea Clerck., Osijek, Damin N. INV.NO.988 (1 M, 11 F): Steatoda bipunctata L., Zemun, 1899., Damin N. INV. NO.1255 (1 JUV* 1 SUBM ${ }^{*}$ ): Teutana castanea Clerck. INV.NO.1261 (1 F): Teutana castanea Clerck., Osijek, 1897., Langhoffer. INV.NO.1284 (1 JUV*, 1 SUBM*, 2 F): Teutana castanea Clerck., Ogulin, 19.04.1896. INV.NO.1290 (2 SUBM*): Teutana castanea Clerck., Topusko. INV.NO.1990 (2 F): Steatoda bipunctata L. Koch, Topusko, 08.1891.

Steatoda grossa (C. L. Koch, 1838)

INV.NO.787 (1 F, 3 JUV*): Teutana grossa C.L. Koch, Zadar, Damin N. INV.NO.788 (3 F): Teutana grossa C.L. Koch, Gasperini. INV.NO.789 (3 F): Teutana grossa C.L. Koch. INV.NO.800 (2 F): Teutana grossa C.L. Koch, Palagruža, 1911. INV.NO.1285 (1 F): Teutana grossa C.L. Koch, Bakar (pivnica), 15.9.1890. INV.NO.1736 (1 M, 1 SUBF*): Teutana grossa C.L. Koch, Bakar (pivnica), 15.09.1890. INV.NO.2389 (1 SUBM ${ }^{*}, 1$ SUBF$^{*}, 10$ JUV$\left.^{*}\right)$ : Teutana grossa C.L. Koch, Bakar (pivnica), 15.9.1890.

Steatoda paykulliana (Walckenaer, 1806)

INV.NO.1288 (1 F): Lithyphantes Paykullianus Walck., 1901. INV.NO.1718 (3 JUV*): Lithyphantes Paykullianus Walck., Budva (Dalm.). INV.NO.34 (1 SUBM*, 1 SUBF): Lithyphantes Paykullianus Walck., Gasperini. INV.NO.35 (3 SUBF*): Lithyphantes Paykullianus Walck., Gasperini. INV.NO.1007 (2 JUV*): Lithyphantes Paykullianus Walck. Fužine, 9.9.1897., Damin. INV.NO.1008 (1 JUV*, 1 SUBM* ${ }^{*} 1$ SUBF*): Lithyphantes Paykullianus Walck., Budva, Damin. INV.NO.1009 (1 JUV*): Lithyphantes Paykullianus Walck., Kotor, Damin.

Steatoda sp.

INV.NO.1087 (1 JUV): Tibellus oblongus Walck., Osijek, 1907. INV.NO.1531 (1 JUV): Epeira Sturmii Hahn., Osijek, 12.3.1898. INV.NO.2370 (1 JUV): Theridium aulicum C.L. Koch.

Steatoda triangulosa (Walckenaer, 1802)

INV.NO.649 (2 F): Teutana triangulosa Walck., Jelenje, Damin N. INV.NO.791 (1 JUV*, 14 F): Teutana triangulosa Walck., Gasperini. INV.NO.792 (1 SUBM*, 11 F): Teutana 
triangulosa Walck., Osijek, Damin N. INV.NO.793 (5 JUV*, 13 F): Teutana triangulosa Walck., Zemun, 15.9.1890., Damin N. INV.NO.794 (2 JUV*, 3 SUBM*, 5 SUBF*, 12 M, 29 F): Teutana triangulosa Walck., Bakar, Primorje, Damin N. INV.NO.795 (7 JUV*, 13 F): Teutana triangulosa Walck., Osijek, Damin N. INV.NO.797 (1 F): Teutana triangulosa Walck., Zadar, Damin N. INV.NO.1256 (1 F): Teutana triangulosa Walck., Zagreb, 18.7.1902. INV.NO.1257 (1 M): Teutana triangulosa Walck., Bakar, 6.8.1902., Polić. INV.NO.1258 (1 F): Teutana triangulosa Walck., Bakar, 29.7.1902. INV.NO.1262 (1 M, 2 JUV*, 2 SUBM*, 2 F): Teutana triangulosa Walck. INV.NO.1270 (1 F): Teutana triangulosa Walck., Lukovo, 9.7.1899. INV.NO.1275 (1 M): Teutana triangulosa Walck., Bakar. INV.NO.1280 (1 M, 1 F): Teutana triangulosa Walck., Borova draga, 28.7.1897. INV.NO.1286 (1 F): Teutana triangulosa Walck., Osijek, 1897., Langhoffer. INV.NO.2324 (1 JUV*): Teutana triangulosa Walck., Jelenje, Damin N. INV.NO.2388 (1 F): Teutana grossa C.L. Koch, Bakar (pivnica), 15.9.1890.

Theridiidae gen. sp.

INV.NO.2318 (1 M): Formicina mutinensis Canestr., Glavotok. INV.NO.2371 (1 JUV): Ergane iucunda Luc., Martinšćica, 14.08.1890., Damin.

Theridion betteni Wiehle, $1960^{*}$

INV.NO.1997 (1 F): Theridium lepidum Walck., Klanjec, 1904., Broz. INV.NO.2302 (1 F): Epeira quadrata Clerck., Zagreb.

Theridion hemerobium Simon, 1914

INV.NO.2323 (1M): Theridium denticulatum Walck., Zemun, 1899., Damin N.

Theridion melanurum Hahn, 1831

INV.NO.636 (1 M, 5 F): Theridium denticulatum Walck., Zemun, 1899., Damin N. INV. NO.637 (3 M, 3 F): Theridium denticulatum Walck., Bakar, 11.5.1899., Damin N. INV. NO.866 (1 F): Theridium denticulatum Walck., Osijek, Zemun, 1.10.1899., Damin N. INV. NO.884 (1 M, 1 F): Theridium denticulatum Walck., Cavtat, Damin N. INV.NO.2436 (1 F): Philodromus margaritatus Clerck., Zemun, 1894. INV.NO.637 (4 JUV*, 4 SUBM $\left.{ }^{*}\right)$ : Theridium denticulatum Walck., Bakar, 11.5.1899., Damin N. INV.NO.638 (1 JUV*): Thomisus albus Gmel. INV.NO.866 (1 JUV*, 3 SUBM $\left.{ }^{*}\right)$ : Theridium denticulatum Walck., Osijek, Zemun, 1.10.1899., Damin N. INV.NO.884 (4 JUV*): Theridium denticulatum Walck., Cavtat, Damin N. INV.NO.1203 (1 JUV*): Theridium denticulatum Walck., Grad Mali. INV.NO.1204 (1 JUV*): Theridium denticulatum Walck., Križišće, 6.8.1893. INV. NO.1251 (1 SUBM ${ }^{*}$ ): Theridium denticulatum Walck., Bakar, 1.03. INV.NO.1714 (3 SUBM*, 21 JUV $^{*}$ ): Theridium denticulatum Walck., Bakar, 20.04.1890. INV.NO.2030 (1 JUV*): Theridium denticulatum Walck., Krk, blizu Sv. Ivana.

Theridion mystaceum L. Koch, $1870^{*}$

INV.NO.632 (1 F): Theridium tepidariorum C.L. Koch, Bakar (na topoli), 25.5., Damin N. INV.NO.1209 (1 F): Theridium denticulatum Walck., Ogulin, 19.4.1896.

Theridion pinastri L. Koch, 1872

INV.NO.629 (1 F): Theridium pinastri L. Koch, Košljun, Damin N.

Theridion $\mathrm{sp}$.

INV.NO.2257 (1 JUV*): Tetragnatha extensa Linn., Osijek, Damin. INV.NO.2379 (1 SUBF): Philodromus margaritatus Clerck., Osijek. 
Theridion varians Hahn, $1833^{* * *}$

INV.NO.1730 (1 JUV*): Theridium varians Hahn., Mrzla vodica.

Theridium pulchellum Walck. ${ }^{* * *}$

INV.NO.214 (2 JUV*): Theridium pulchellum Walck., Porto Palazzo, Damin N.

\section{Thomisidae Sundevall, 1833}

Coriarachne depressa (C. L. Koch, 1837)

INV.NO.165 (1 JUV*): Coriarachne depressa C.L. Koch, Bakar.

Diaea dorsata (Fabricius, 1777)

INV.NO.13 (2 M, 8 JUV$\left.^{*}\right)$ : Diaea dorsata Fabr., Polača, Damin. INV.NO.14 (1 M, 1 F, 2 JUV*): Diaea dorsata Fabr., Bakar, Damin. INV.NO.782 (1 F): Diaea dorsata Fabr., Fužine, 6.1897. INV.NO.992 (1 JUV*): Diaea dorsata Fabr. INV.NO.993 (1 JUV*, 1 F): Diaea dorsata Fabr., Pod Bitorajem. INV.NO.1976 (1 JUV*): Diaea dorsata Fabr., Unčica, 28.08.1911.

Ebrechtella tricuspidata (Fabricius, 1775)

INV.NO.738 (1 JUV*, 2 M, 4 F): Misumena tricuspidata Fabr., Zemun, 1899., Damin. INV.NO.739 (1 F, 2 SUBM$^{*}, 5$ JUV$\left.^{*}, 10 \mathrm{M}\right)$ : Misumena tricuspidata Fabr., Damin. INV. NO.740 (1 JUV*): Misumena tricuspidata Fabr., Skradin, Damin. INV.NO.741 (1 JUV*, 6 M): Misumena tricuspidata Fabr., Zagreb, 1895., Damin. INV.NO.781 (3 SUBM*, 4 JUV$^{*}$ ): Misumena tricuspidata Fabr., Topusko. INV.NO.783 (1 JUV*, $2 \mathrm{M})$ : Misumena tricuspidata Fabr., Križevci, 4.9.1897. INV.NO.818 (1 JUV*): Misumena tricuspidata Fabr., Zagreb, 1897. INV.NO.958 (2 JUV*, 3 M): Misumena tricuspidata Fabr., Osijek, 12.1898. INV.NO.1072 (1 M): Misumena tricuspidata Fabr., Osijek, 1907. INV.NO.1283 (1 F): Misumena tricuspidata Fabr., Boljevci-zidine, 26.5.1912. INV.NO.1815 (1 F): Misumena tricuspidata Fabr., Žaver, 28.05.1905. INV.NO.1956 (1 M): Misumena tricuspidata Fabr., Mošćenice, 01.09.1911. INV. NO.2325 (1 M): Oxyptila horticola C.L. Koch, Bakar, Damin.

Heriaeus hirtus (Latreille, 1819)

INV.NO.620 (2 F): Heriaeus Savignyi E. Sim., Bakar, Damin. INV.NO.621 (1 F): Heriaeus Savignyi E. Sim., Zadar, Damin. INV.NO.627 (1 M): Heriaeus setiger Cambr., Gasperini. INV.NO.628 (1 M): Heriaeus setiger Cambr., Gasperini. INV.NO.928 (1 F, 6 JUV*): Heriaeus Savignyi E. Sim., Bakar. INV.NO.957 (1 F): Heriaeus Savignyi E. Sim., Zadar, 25.7.1899. INV.NO.1602 (1 JUV*, 1 M, 2 SUBF*): Heriaeus Savignyi E. Sim., Kotor. INV.NO.1716 (1 $J_{U V}^{*}$ ): Heriaeus Savignyi E. Sim., Cavtat. INV.NO.619 (1 JUV*): Heriaeus Savignyi E. Sim., Gasperini. INV.NO.620 (1 SUBM*, 2 SUBF*, 8 JUV*): Heriaeus Savignyi E. Sim., Bakar, Damin. INV.NO.621 (1 JUV*): Heriaeus Savignyi E. Sim., Zadar, Damin. INV.NO.622 (1 $\left.J_{U V}^{*}\right)$ : Heriaeus Savignyi E. Sim., Kotor, Damin. INV.NO.623 (1 JUV*): Heriaeus Savignyi E. Sim., Tajar, 1.3. INV.NO.625 (1 SUBM*): Heriaeus hirsutus Walck., Jablanac, Damin.

Heriaeus oblongus Simon, 1918

INV.NO.624 (1 F): Heriaeus hirsutus Walck., Kotor, Muo, Damin. INV.NO.625 (1 F): Heriaeus hirsutus Walck., Jablanac, Damin. INV.NO.1743 (1 F): Heriaeus hirsutus Walck., Kotor.

Heriaeus sp.

INV.NO.754 (1 JUV): Pisaura mirabilis CI., Novi Vinodol, 15.4.1897. INV.NO.980 (1 JUV): Heriaeus sp. 
Misumena vatia (Clerck, 1757)

INV.NO.684 (1 F): Misumena vatia Clerck., Gračani, 13.6.1901. INV.NO.704 (1 M, 1 SUBM$^{*}, 2$ F): Misumena vatia Clerck., Osijek, 7.1901., Rössler. INV.NO.735 (1 JUV*): Misumena vatia Clerck. INV.NO.737 (1 M, 1 F): Misumena vatia Clerck., Vrana, Damin. INV.NO.755 (1 F): Misumena vatia Clerck., Plitvice, 27.8.1902. INV.NO.835 (2 F): Misumena vatia Clerck., Osijek, 1907., Langhoffer. INV.NO.836 (1 SUBF*): Misumena vatia Clerck., Ogulin, 4.6.1898. INV.NO.853 (1 F): Thomisus albus Gmel., Kotor, Damin. INV.NO.900 (1 SUBF*): Misumena vatia Clerck., Osijek, 7.1901., Rössler. INV.NO.901 (1 F): Misumena vatia Clerck., Fužine, 6.1897. INV.NO.941 (1 F): Misumena vatia Clerck., Križevci, 14.4.1897. INV.NO.1070 (2 F): Misumena tricuspidata Fabr., Osijek, 7.1901., Rössler. INV.NO.1708 (1 M): Misumena vatia Clerck., Kraljičin zdenac, 14.06.1897., Langhoffer. INV.NO.1799 (1 JUV*): Misumena vatia Clerck., Zdenčina, 20.08.1912. INV.NO.1804 (1 F): Misumena vatia Clerck., Remete, 29.07.1917. INV.NO.1810 (2 F): Misumena vatia Clerck., Šišatovac, 25.07.1917. INV.NO.1819 (1 SUBF*): Misumena vatia Clerck., Kupinovo, 20.07.1902. INV. NO.1821 (1 M): Tmarus piger Walck., Sikirevci, 16.07.1912. INV.NO.1970 (1 F): Misumena tricuspidata Fabr., Viljevo, 18.08.1915. INV.NO.1972 (1 F): Misumena vatia Clerck., Babina greda, 17.07.1912. INV.NO.1974 (1 F): Misumena tricuspidata Fabr., Turopolje, 21.06.1914. INV.NO.2348 (1 M, 3 F): Runcinia lateralis C.L. Koch, Bakar. INV.NO.2367 (2 F): Thomisus albus Gmel., Bakar, 10.5.1890., Damin. INV.NO.2456 (1 SUBM*): Misumena vatia Clerck., Topusko, 08.08.1898.

Ozyptila atomaria (Panzer, 1801)***

INV.NO.694 (1 SUBF*): Oxyptila horticola C.L. Koch, Šibenik, Damin. INV.NO.695 (2 $\mathrm{JUV}^{*}$ ): Oxyptila horticola C.L. Koch, Bakar, Damin.

Ozyptila claveata (Walckenaer, 1837)

INV.NO.693 (1 M, 1 F): Oxyptila nigrita Thor., Senj, Damin.

Ozyptila confluens (C. L. Koch, 1845)

INV.NO.306 (4 F): Ozyptila horticola C.L. Koch, Gasperini. INV.NO.861 (1 F): Philodromus lividus Sim., Gasperini.

Ozyptila praticola (C. L. Koch, 1837)

INV.NO.697 (1 JUV*, 1 F): Oxyptila praticola C.L. Koch, Bakar, Damin. INV.NO.1040 (1 F): Oxyptila praticola C.L. Koch, Zemun, 1897. INV.NO.1045 (1 SUBM*): Oxyptila praticola C.L. Koch, Osijek, 4.1899. INV.NO.1051 (1 JUV*): Oxyptila praticola C.L. Koch, Topusko, 8.1891 .

Ozyptila sp.

INV.NO.2402 (1 JUV): Epeira patagiata Clerck., Fužine, 06.06.1897., Dr. Langhoffer.

Pistius truncatus (Pallas, 1772)

INV.NO.16 (1 SUBM* , 2 F, 4 SUBF$^{*}, 6$ JUV$\left.^{*}\right)$ : Pistius truncatus Pallas., Sv. Kuzma (cesta), 14.08.1890., Damin. INV.NO.616 (1 F): Pistius truncatus Pallas., Plitvice. INV.NO.639 (1 $J_{U}^{*}$ ): Pistius truncatus Pallas., Skradin. INV.NO.742 (1 SUBF*): Pistius truncatus Pallas., Bribir-Novi, 15.04.1897. INV.NO.767 (2 JUV*): Pistius truncatus Pallas., Topusko, 08.1891. INV.NO.768 (2 JUV*): Pistius truncatus Pallas., Bribir, 1901. INV.NO.2455 (1 JUV*): Pistius truncatus Pallas. INV.NO.609 (1 JUV*): Pistius truncatus Pallas., Zagreb, Sofijin put. 
Runcinia grammica (C. L. Koch, 1837)

INV.NO.762 (4 F): Runcinia lateralis C.L. Koch, Kotor, Muo, Damin. INV.NO.763 (1 M, 2 SUBM $^{*}, 2$ SUBF$^{*}, 9$ F, 11 JUV$^{*}$ ): Runcinia lateralis C.L. Koch, Bakar, Damin. INV.NO.764 (1 SUBF*, 4 F): Runcinia lateralis C.L. Koch, Bakar. INV.NO.765 (2 F): Runcinia lateralis C.L. Koch, Gasperini. INV.NO.819 (1 JUV*): Runcinia lateralis C.L. Koch. INV.NO.883 (3 F): Runcinia lateralis C.L. Koch, Borova draga, 8.7., Gery. INV.NO.942 (4 JUV*): Runcinia lateralis C.L. Koch. INV.NO.959 (3 F): Runcinia lateralis C.L. Koch, Bakar, 10.1891. INV. NO.983 (1 F): Runcinia lateralis C.L. Koch, Crikvenica, 8.12. INV.NO.984 (2 F): Runcinia lateralis C.L. Koch, Rovinj (Istra), 23.9.1902. INV.NO.994 (1 JUV*): Runcinia lateralis C.L. Koch, Lukovo, 7.7.1899. INV.NO.1603 (1 F): Runcinia lateralis C.L. Koch, Topusko. INV. NO.1802 (2 JUV*): Runcinia lateralis C.L. Koch, Bakar, 01.06.1905. INV.NO.1806 (2 F): Runcinia lateralis C.L. Koch, Rab, 16.07.1908., K. Babić. INV.NO.1818 (1 M, 2 F): Runcinia lateralis C.L. Koch, Bakar, 06.08.1905., Polić. INV.NO.1824 (1 JUV*): Runcinia lateralis C.L. Koch, Stari grad (Dalm.). INV.NO.2021 (1 F): Runcinia lateralis C.L. Koch, Osijek, 07.1901., Rössler.

Synema cf. ornatum sensu Utochkin, 1960 (Thorell, 1875)*

INV.NO.747 (1 F): Synaema ornatum Thor., Lokrum, Damin.

Synema cf. plorator sensu Levy, 1985 (O. P.-Cambridge, 1872)

INV.NO.2340 (1 F): Synaema globosum F., Gasperini.

Synema globosum (Fabricius, 1775)

INV.NO.744 (2 F): Synaema globosum F., Bakar, Damin. INV.NO.745 (2 F): Synaema globosum F., Novigrad, Damin. INV.NO.746 (3 M): Synaema globosum F., Gasperini. INV.NO.882 (1SSP*): Synaema globosum F., Jasikovac, 18.8.1902. INV.NO.897 (1JUV*): Synaema globosum F., Lokrum. INV.NO.927 (1 M): Synaema globosum F., Osijek, 1897., Langhoffer. INV.NO.956 (1 JUV*): Synaema globosum F., Topusko, 8.1891. INV.NO.1800 (2 F): Xysticus lateralis Hahn., Božjakovina, 16.08.1915. INV.NO.1960 (1 F): Xysticus cristatus Clerck., Maksimir, 18.05.1908. INV.NO.2341 (3 JUV*): Synaema globosum F., Gasperini.

Synema sp.

INV.NO.979 (2 JUV): Synaema sp., Starigrad.

Thomisidae gen. Sp.

INV.NO.2399 (2 JUV): Epeira Sturmii Hahn., Osijek, 1897., Dr. Langhoffer.

Thomisus onustus Walckenaer, 1805

INV.NO.610 (3 F): Thomisus albus Gmel., Gračani, 13.6.1901. INV.NO.611 (1 M): Thomisus albus Gmel. INV.NO.615 (3 F): Thomisus albus Gmel., Bakar. INV.NO.766 (1 F, 2 M): Thomisus albus Gmel., Borova draga, 08.06., S. Jezi. INV.NO.850 (1 M, 3 F): Thomisus albus Gmel., Bakar, 10.5.1890., Damin. INV.NO.851 (1 M, 1 F): Thomisus albus Gmel., Vis, Damin. INV.NO.852 (3 F): Thomisus albus Gmel., Bakar, Damin. INV.NO.925 (1 M): Thomisus albus Gmel., Jablanac. INV.NO.1796 (2 M, 3 F): Thomisus albus Gmel., Bakar. INV.NO.1797 (1 F): Thomisus albus Gmel., Kotor, 1.6.1908. INV.NO.1811 (1 F): Thomisus albus Gmel., Đurđevac, 03.07.1913. INV.NO.1812 (1 F): Thomisus albus Gmel., Kotor, 29.05.1908., K. Babić. INV.NO.2109 (1 F): Thomisus albus Gmel., Caska, 09.06.1958. INV. NO.2194 (1 F): Misumena calycina L. Koch, Velo blato, o. Pag, 04.07.1956. INV.NO.2243 (1 M): Pistius truncatus Pallas., Sv. Kuzma (cesta), 14.08.1890., Damin. 
Thomisus sp.

INV.NO.582 (1 JUV*, 1 SUBM*): Thomisus albus Gmel. INV.NO.583 (1 JUV*): Thomisus albus Gmel., Rovinj, 23.9.1902. INV.NO.736 (1 SUBF*): Thomisus albus Gmel., Zemun. INV.NO.766 (7 JUV*): Thomisus albus Gmel., Borova draga, 08.06., S. Jezi. INV.NO.850 (3 SUBF*, 9 JUV*): Thomisus albus Gmel., Bakar, 10.5.1890., Damin. INV.NO.923\#: Thomisus albus Gmel. INV.NO.924 (1 SUBM*): Thomisus albus Gmel., Pelagosa, 1911., Dr. Babić. INV.NO.925 (1 SUBF*): Thomisus albus Gmel., Jablanac. INV.NO.1214 (1 JUV*): Thomisus albus Gmel., Stari grad, 29.7. INV.NO.2185 (1 SUBF*): Thomisus albus Gmel., Susak, 21.06.1962. INV.NO.2454 (1 JUV*): Thomisus albus Gmel., Rijeka, 23.09.1897. INV.NO.1453 $\left(1 \mathrm{JUV}^{*}\right)$ : Filistata insidiatrix Forsk., Bakarac, 24.08.1889. INV.NO.2224\#: Thomisus albus Gmel., Vermač, 07.1931. INV.NO.2410 (1 JUV*): Philodromus aureolus rufolimbatus Clerck verus, Bribir-Novi, 15.04.1897.

Tmarus piger (Walckenaer, 1802)

INV.NO.294 (3 M, 4 SUBF*, 5 F, 8 SUBM $^{*}$, 14 JUV*): Tmarus piger Walck., Damin. INV. NO.295 (1 SUBF*, 2 F): Tmarus piger Walck., Damin. INV.NO.995 (2 JUV*): Tmarus piger Walck., Topusko, 8.1891. INV.NO.1004 (1 M, 1 F): Tmarus piger Walck., Dol-Grižane. INV. NO.1023 (1 JUV*): Tmarus piger Walck. INV.NO.1054 (1 M): Tmarus piger Walck., Osijek, 04.1899. INV.NO.2383 (5 JUV*): Tmarus piger Walck.

Tmarus stellio Simon, 1875

INV.NO.1035 (1 SUBM*): Tmarus stellio, Dol-Grižane, 14.04. INV.NO.1039 (1 F): Tmarus stellio, Zagreb, 1908. INV.NO.1825 (1 JUV*): Tmarus stellio, Lokrum.

Xysticus acerbus Thorell, 1872

INV.NO.81 (1 M, 3 F): Xysticus acerbus Thor., Bakar, 29.05., Damin. INV.NO.82 (1 M, 1 SUBM$^{*}, 2$ JUV$\left.^{*}\right)$ : Xysticus acerbus Thor., Gasperini. INV.NO.817 (1 M): Xysticus acerbus Thor., Grižane, 14.4. INV.NO.869 (1 M): Xysticus acerbus Thor., Bribir-Novi, 15.4.1897. INV.NO.991 (1 F): Xysticus acerbus Thor. INV.NO.1969 (1 F): Tibellus parallelus C.L. Koch, Zagreb, 1901. INV.NO.2253 (1 F, 2 M): Xysticus Kochii Thor., Buk Krke, Damin.

Xysticus apricus L. Koch, 1876

INV.NO.1037 (1M): Xysticus marmoratus Thor., Rijeka, 23.9.1897.

Xysticus audax (Schrank, 1803)

INV.NO.552 (1 M, 2 F): Xysticus pini Hahn., Osijek, Zemun, Damin. INV.NO.1032 (1 F): Xysticus ulmi Hahn., Zagreb, 1897. INV.NO.1056 (1 F): Xysticus pini Ihahn., Fužine, 6.1897. INV.NO.1713 (1 F): Xysticus pini Hahn., Zemun, 1899. INV.NO.1052 (1JUV*): Xysticus pini Hahn., Zagreb, 1897. INV.NO.2413 (1 JUV*): Xysticus pini Hahn., Zemun, 1899.

Xysticus bifasciatus C. L. Koch, $1837^{*}$

INV.NO.672 (1 M, 1 F): Xysticus bifasciatus C.L. Koch, Damin. INV.NO.926 (1 F): Xysticus bifasciatus C.L. Koch, Fužine, 6.1897. INV.NO.1809 (2 F): Tibellus parallelus C.L. Koch, Sv. Gera, 19.06.1917. INV.NO.1953 (2 JUV*): Xysticus bifasciatus C.L. Koch, Mitrovica, 25.04.1911.

Xysticus bufo (Dufour, 1820)

INV.NO.1036 (1 F): Oxyptila sp., Gasperini. INV.NO.2144 (1 F): Xysticus sp., Novalja, 01.10.1957. 
Xysticus cor Canestrini, 1873

INV.NO.284 (1 F): Xysticus comptulus Sim., Novigrad, Damin. INV.NO.285 (1 M): Xysticus comptulus Sim., Bakar, 3.4., Damin. INV.NO.286 (1 M): Xysticus comptulus Sim., Sv. Duh, 26.3.1889., Damin. INV.NO.287 (1 F): Xysticus comptulus Sim., Gasperini. INV. NO.377 (1 F): Xysticus cristatus Clerck., Gasperini. INV.NO.896 (1 F): Xysticus comptulus Sim., Senj. INV.NO.953\#: Xysticus comptulus Sim., Bakar. INV.NO.960 (1 F): Xysticus Kochii Thor., Senj. INV.NO.2051 (2 F): Xysticus comptulus Sim.,

Xysticus cristatus (Clerck, 1757)

INV.NO.376 (1 M, 1 F): Xysticus cristatus Clerck., Risnjak, Damin. INV.NO.778 (1 M): Xysticus cristatus Clerck., Kecskemet, 3.1890., Salzteid. INV.NO.838 (1 M): Xysticus cristatus Clerck., Bribir-Novi, 15.4.1897. INV.NO.977 (1 M, 2 F): Xysticus cristatus Clerck., Fužine, 7.1897. INV.NO.1954 (1 M): Xysticus cristatus Clerck., Lipa, 04.06.1899.

Xysticus erraticus (Blackwall, 1834)*

INV.NO.837 (1 M): Xysticus erraticus Blackw., Zagreb, 18.7.1902. INV.NO.895\#: Xysticus erraticus Blackw., Zagreb, 7.9.1902. INV.NO.2377 (1 M): Xysticus gallicus E. Sim., Delnice.

Xysticus gallicus Simon, $1875^{*}$

INV.NO.919 (2 M): Xysticus gallicus E. Sim., Delnice. INV.NO.1975 (1 F): Tibellus parallelus C.L. Koch, Bitoraj, 28.06.1910.

\section{Xysticus graecus C. L. Koch, 1837}

INV.NO.943 (1 F): Xysticus graecus C.L. Koch, Pad Krke. INV.NO.949 (1 JUV*, 1 SUBM$^{*}$, 1 F): Xysticus graecus C.L. Koch, Bakar, Damin. INV.NO.950 (1 SUBM*): Xysticus graecus C.L. Koch, Selce, Damin. INV.NO.951 (1 JUV*): Xysticus graecus C.L. Koch, Kotor, Damin. INV.NO.952 (1 SUBM*): Xysticus graecus C.L. Koch, Gasperini. INV.NO.976 (1 JUV*, 1 F): Xysticus graecus C.L. Koch., Bakar. INV.NO.1071 (1 F): Misumena tricuspidata Fabr., Pad Krke. INV.NO.1701 (1 F): Xysticus graecus C.L. Koch, Lukovo, 17.07. INV.NO.1721 (1 SUBF*, 1 F, 2 JUV*): Xysticus graecus C.L. Koch, Senj, 04.07.1899. INV.NO.1955 (1 M): Xysticus graecus C.L. Koch, Sv. Juraj, 17.07. INV.NO.2241 (1 $\left.\mathrm{M}^{*}\right)$ : Xysticus graecus C.L. Koch, Lokve, 07.1930.

\section{Xysticus kempeleni Thorell, $1872^{* * *}$}

INV.NO.671 (1JUV*): Xysticus Kempelenii Thor., Bakar (Podstene), 07.08.1889., Damin.

\section{Xysticus kochi Thorell, 1872}

INV.NO.83 (2 JUV*, 2 F, 5 M): Xysticus Kochii Thor., Buk Krke, Damin. INV.NO.84 (2 F): Xysticus Kochii Thor., Ratulje, Damin. INV.NO.85 (1 F): Xysticus Kochii Thor., Gasperini. INV.NO.551 (5 F): Xysticus pini Hahn., Fužine, Damin. INV.NO.554 (1 F): Xysticus lateralis Hahn., Ogulin, Damin. INV.NO.557 (1 F): Xysticus lateralis Hahn., 19.9.1890., Damin. INV. NO.985 (1 F): Xysticus Kochii Thor., Osijek, 1907., Langhoffer. INV.NO.986 (1 F): Xysticus Kochii Thor., Osijek, 07.1901., Rössler. INV.NO.989 (1 M, 1 F): Xysticus Kochii Thor. INV. NO.990 (1 F): Xysticus Kochii Thor., Božjakovina, 28.5.1898. INV.NO.1021 (1 M): Xysticus Kochii Thor., Zemun, 1891. INV.NO.1022 (1 M): Xysticus Kochii Thor. INV.NO.1034 (1 M, 1 F): Xysticus Kochii Thor., Bakar. INV.NO.1038 (1 F): Xysticus Kochii Thor., Plitvice, 27.08.1902. INV.NO.1043 (2 M): Xysticus Kochii Thor., Trgovčević. INV.NO.1798 (1 F): Tibellus parallelus C.L. Koch, Mitrovica, 27.05.1911. INV.NO.1808 (1 F): Xysticus robustus Hahn., Jarak, 23.07.1917. INV.NO.1822 (1 F): Tibellus parallelus C.L. Koch, Čeravić, 30.05.1910. INV.NO.1978 (1 F): Tibellus parallelus C.L. Koch, Turopolje, 21.06.1914. INV. NO.2314 (2 F): Xysticus pini Hahn., Osijek, Zemun, Damin. INV.NO.2412 (4 F): Xysticus pini Hahn., Zemun, 1899. 
Xysticus lanio C. L. Koch, 1835

INV.NO.553 (1 JUV*, $1 \mathrm{M})$ : Xysticus lateralis Hahn., Košljun, o. Krk, Damin. INV. NO.555 (1 M, 1 F): Xysticus lateralis Hahn, Kotor, Damin. INV.NO.556 (2 JUV*): Xysticus lateralis Hahn., Damin. INV.NO.1011 (1 JUV*, 2 SUBF*): Xysticus lateralis Hahn., Bakar, 27.7.1902. INV.NO.1033 (1 JUV*): Xysticus lateralis Hahn., Fužine, 6.6.1897. INV.NO.1048 (1 SUBF*): Xysticus lateralis Hahn. INV.NO.1049 (1 M): Xysticus lateralis Hahn., Izvor Rječine, 22.5.1890. INV.NO.1053 (8 JUV*): Xysticus lateralis Hahn. INV.NO.1069 (1 JUV*): Xysticus lateralis Hahn., Sofijin put (Zagreb), 19.6.1896. INV.NO.1431 (3 JUV*): Xysticus lateralis Hahn., Kotor, 3.8.1914. INV.NO.1963 (1 F): Xysticus lateralis Hahn., Ratulje.

Xysticus luctator L. Koch, 1870

INV.NO.2033 (2 F): Xysticus luctator.

Xysticus ninnii Thorell, 1872

INV.NO.656 (1 M, 2 JUV*, 2 F): Xysticus Ninnii Thor., Jablanac, Senj, Damin. INV.NO.657 (1 SUBF*): Xysticus Ninnii Thor., Vis, Damin. INV.NO.658 (2 JUV*): Xysticus Ninnii Thor., Bakar, Damin. INV.NO.1042 (1 SUBF*): Xysticus Ninnii Thor., Zadar, 23.7.1899. INV. NO.1047 (1 JUV*): Xysticus Ninnii Thor., Fužine, 6.6.1897. INV.NO.1813 (1 M): Tibellus parallelus C.L. Koch, Biševo, 24.07.1917.

Xysticus robustus (Hahn, 1832)

INV.NO.673 (1 F): Xysticus robustus Hahn., Bakar, 9.8., Damin. INV.NO.674 (1 JUV*): Xysticus robustus Hahn., Gasperini. INV.NO.1807 (1 M): Xysticus sp., Starigrad (Kekići), 24.06.1917. INV.NO.1814 (1 F): Xysticus robustus Hahn., Stari grad, o. Hvar, 09.07.1894. INV.NO.1973 (1 F): Xysticus robustus Hahn., Buk Krke. INV.NO.2106 (1 F): Oxyptila sp., Caska, o. Pag, 12.06.1958.

Xysticus sp.

INV.NO.918 (2 JUV): Xysticus sp., Križevci, 4.9.1897. INV.NO.1005 (1 JUV, 1 SUBF): Xysticus sp., Fužine, 6.1897. INV.NO.1006 (1 JUV): Xysticus sp., 1902., Broz. INV.NO.1041 (2 JUV): Xysticus sp., Osijek, 1897., Langhoffer. INV.NO.1046 (4 JUV): Xysticus sp., Rovinj, 23.9.1902. INV.NO.1055 (1 SUBM): Xysticus sp., Zagreb, 1897. INV.NO.1068 (1 JUV): Xysticus sp., Stara Pazova, 10.8.1898. INV.NO.1071 (2 JUV): Misumena tricuspidata Fabr., Pad Krke. INV.NO.1813 (5 JUV): Tibellus parallelus C.L. Koch, Biševo, 24.07.1917. INV. NO.1817 (1 JUV*): Tibellus parallelus C.L. Koch, Zdenčina, 20.08.1912. INV.NO.1820 (1 JUV): Tibellus parallelus C.L. Koch, Boljevci-zidine, 21.05.1912.

Xysticus striatipes L. Koch, 1870

INV.NO.667 (1 M, 1 SUBM*, 1 F): Xysticus striatipes L. Koch, Vis, Damin. INV.NO.668 (2 JUV*): Xysticus striatipes L. Koch, Križišće, Damin. INV.NO.669 (2 M, 2 F): Xysticus striatipes L. Koch, Osijek, Damin. INV.NO.1823 (2 M): Xysticus striatipes L. Koch, Osijek. INV.NO.670 (1 JUV*): Xysticus striatipes L. Koch, Gasperini.

Xysticus tenebrosus Silhavy, $1944^{*}$

INV.NO.2252 (1M): Xysticus acerbus Thor., Bakar, 29.05., Damin.

Xysticus ulmi (Hahn, 1831)

INV.NO.655 (2 JUV*, 3 F): Xysticus ulmi Hahn., Zagreb, Damin. INV.NO.1010 (1 M): Xysticus ulmi Hahn., Božjakovina, 28.5.1898. INV.NO.1032 (1 SUBM $\left.^{*}\right)$ : Xysticus ulmi Hahn., Zagreb, 1897. 
Titanoecidae Lehtinen, 1967

Titanoeca quadriguttata (Hahn, 1833)

INV.NO.299 (1 JUV*): Titanoeca Schineri L. Koch. INV.NO.400 (3 F): Titanoeca Kochii Auss. INV.NO.401 (1 F): Titanoeca Kochii Auss., Split, Damin N. INV.NO.2047 (1 M, 3 $\left.\mathrm{SUBM}^{*}\right)$ : Titanoeca quadriguttata Hahn.

Titanoeca schineri L. Koch, 1872

INV.NO.299 (1 M, 2 F): Titanoeca Schineri L. Koch.

Titanoeca sp.

INV.NO.1175 (1 SUBM): Titanoeca sp., Malinska, 27.3. INV.NO.1180 (5 JUV): Titanoeca sp. INV.NO.1180 (8 SUBM): Titanoeca sp.

Titanoeca tristis L. Koch, 1872

INV.NO.402 (1 SUBM*, 4 F): Titanoeca tristis L. Koch, Bakar, 12.8., Damin N. INV. NO.403 (1 F, 2 JUV $^{*}, 4$ SUBM$\left.^{*}\right)$ : Titanoeca tristis Koch. INV.NO.1171 (1 F): Titanoeca tristis L. Koch, Osijek, 1899. INV.NO.1711 (1 SUBM*, 4 F): Titanoeca tristis L. Koch, Bakar. INV. NO.2032 (5 JUV*): Titanoeca tristis L. Koch, Bakar.

\section{Trachelidae Simon, 1897}

Paratrachelas maculatus (Thorell, 1875)

INV.NO.369 (1 M, 2 JUV*): Trachelas maculatus Thorell, Starigrad, o. Hvar. INV.NO.371 (1 JUV*): Trachelas maculatus Thorell. INV.NO.372 (1 JUV*): Trachelas maculatus Thorell. INV.NO.373 (1SSP*): Trachelas maculatus Thorell, Omišalj. INV.NO.2405 (1 F): Epeira Victoria Thor., Vinkovci.

\section{Uloboridae Thorell, 1869}

Hyptiotes paradoxus (C. L. Koch, 1834)

INV.NO.155 (3 JUV*): Hyptiotes paradoxus C.L. Koch, Kotor, Muo, Damin N. INV. NO.156 (1 M, 1 F, 3 JUV*): Hyptiotes paradoxus C.L. Koch, Bakar (grm), 14.8.1890., Damin N. INV.NO.1186 (1 F): Hyptiotes anceps Walckenaer, Rijeka, 29.08.1897. INV.NO.1680 (1 $J_{U V}^{*}$ ): Hyptiotes paradoxus C.L. Koch, Polača (o. Mljet). INV.NO.1727 (4 JUV*): Hyptiotes paradoxus C.L. Koch, Košljun.

Hyptiotes sp.

INV.NO.2260 (1 JUV): Zilla litterata Olv., Kotor, Damin.

Uloborus plumipes Lucas, 1846

INV.NO.42 (3 F): Uloborus plumipes Lucas, Starigrad, 11.07.1899., Damin. INV.NO.43 (2 F): Uloborus plumipes Lucas, Pećina (Vrana), 23.07., Damin. INV.NO.2000 (3 F): Uloborus plumipes Lucas, Starigrad.

Uloborus walckenaerius Latreille, 1806

INV.NO.36 (1 JUV*): Uloborus Walckenaeri Latr., Komiža (Vis), Damin. INV.NO.37 (1 JUV*, 1 M, 2 F): Uloborus Walckenaeri Latr., Bakar, 27.06.1893., Damin. INV.NO.38 (1 M): Uloborus Walckenaeri Latr., Kotor, Damin. INV.NO.39 (1 F): Uloborus Walckenaeri Latr., Bakar (Srebrač), Damin. INV.NO.40 (2 M, 2 F, 402 JUV*): Uloborus Walckenaeri Latr., Bakar (Srebrač), 07.09., Damin. INV.NO.41 (2 cocoon*): Uloborus Walckenaeri Latr., Bakar (Sojići), 27.06.1893., Damin. INV.NO.1196 (1SSP*): Uloborus Walckenaeri Latr., Bakar, 10.07. INV.NO.1999 (1 F): Uloborus Walckenaeri Latr., Buk Krke. 


\section{Zodariidae Thorell, 1881}

Zodarion elegans (Simon, 1873)

INV.NO.17 (1 F): Zodarium graecum. INV.NO.199 (1 M, 4 F): Zodarium germanicum C.L. Koch, Damin N. INV.NO.263 (2 F): Zodarium germanicum C.L. Koch, Karlobag, 13.7.1899., Damin N. INV.NO.1342 (1SSP*): Zodarium elegans E. Sim., Bag, 7.9.1899. INV. NO.2391 (1SSP*): Zodarium elegans E. Sim., Bag, 7.9.1899.

Zodarion germanicum (C. L. Koch, 1837)*,***

INV.NO.199 (1 JUV*, 1 SUBM $^{*}$ ): Zodarium germanicum C.L. Koch, Damin N.

Zodarion hamatum Wiehle, 1964

INV.NO.2278 (6 M): Zodarium italicum Can. Et PaV., Bakar, Omišalj, Damin N. INV. NO.2417 (1 M): Zodarium italicum, Bakar.

Zodarion italicum (Canestrini, 1868)

INV.NO.262 (4 M): Zodarium italicum Can. Et PaV., Bakar, Omišalj, Damin N. INV. NO.1734 (1 F): Zodarium italicum, Bakar. INV.NO.1938 (1 JUV*, 1 F): Zodarium italicum, Bakar.

\section{Zoropsidae Bertkau, 1882}

Zoropsis oertzeni Dahl, 1901

INV.NO.496 (2 F): Zoropsis ocreata, Bakar, 25.09.-31.10., Damin N. INV.NO.498 (1 M, 1F): Zoropsis ocreata. INV.NO.1950 (2 M): Zoropsis ocreata, Bakar. INV.NO.495 (1 JUV*): Zoropsis ocreata, Šibenik, Damin N. INV.NO.496 (8 JUV*): Zoropsis ocreata, Bakar, 25.09.31.10., Damin N.

Zoropsis spinimana (Dufour, 1820)

INV.NO.497 (2 F): Zoropsis ocreata, Damin N. INV.NO.500 (1 F): Zoropsis lutea Thor., Gasperini.

\section{Opiliones}

INV.NO.567 (3SSP): Trochosa leopardus Sund.Kraljičin zdenac, 18.11.1901. INV. NO.1419 (2 JUV*): Dysdera sp., dr. Langhoffer.)

\section{ACKNOWLEDGEMENT}

We would like to thank all the colleague-arachnologists (Jerzy Prószyński, Petr Dolejš, Yuri Marusik, Tanya Tuneva, Peter van Helsdingen, Milan Rezác, Mykola Kovblyuk, Fulvio Gasparo, Arthur Decae, Angelo Bolzern and many others) who provided us with invaluable help with species identification and the literature needed. For their frequently needed "second opinion" we are also in debt to members of the Croatian Arachnological Society "Narcis Damin".

We thank Darija Ćaleta and Marina Višić Vranjković for their help in documentation and literature survey. We also wish to thank Marijana Vuković for her kind help in proofreading of the ms and Mladen Kučinić for clarifying the ideas discussed in it.

Finally, the authors thank two reviewers for their helpful feedback on an earlier draft. 


\section{REFERENCES}

BACCAlONI, J., 2012: A Preliminary Comparison of Trisodium Phosphate with Agepon and Decon90 as Wetting Agents to Hydrate Dried Arachnida and Myriapoda Specimens. NatSCA News 22, 71-79.

Bosmans, R., Henrard, A., Benhalima, S. \& Kherbouche-Abrous, O., 2017: The genus Clubiona Latreille, 1904 (Araneae: Clubionidae) in the Maghreb, with notes on the genevensis group and new records from the Mediterranean Region. Zootaxa 4353(1), 1-28.

Buchar, J. \& Thaler, K., 1997: Die Wolfspinnen von Österreich 4 (Schluß): Gattung Pardosa max. p. (Arachnida, Araneae: Lycosidae) - Faunistisch-tiergeographische Übersicht *). Carinthia II, 187, 515-539.

Chyzer, K. \& Kulczyński, W., 1894: Araneae Hungariae, Tomus II - di pars prior (Vol. 2). Budapest: Acad. Sci. Hung.

Chyzer, K. \& Kulczyński, W., 1897: Araneae Hungariae, Tomus II - di pars posterior (Vol. 2). Budapest: Acad. Sci. Hung.

Čanađija, S., 1974. Hrvatski narodni zoološki muzej od osnutka do danas: In MatoničKIN, I. (ur.): Spomenica: sto godina znanstvenog I nastavnog rada iz zoologije na Sveučilištu u Zagrebu. Zagreb, Zoologijski zavod Prirodoslovno - matematičkog fakulteta Sveučilišta u Zagrebu.

Čanađija, S. \& J. PAvletić, 1976: Vodič : Hrvatski narodni zoološki muzej / [ur. S. Čanađija \& J. Pavletić; fotografije K. Igalffy]. - 2. izd - Zagreb : Hrvatski narodni zoološki muzej, 1976. - 43 str. : ilustr.; $24 \mathrm{~cm}$

Damin, N., 1896: Gnaphosa Kulczynskii nov. sp. Soc. Hist. - Nat. Croat. 9, 343-345.

Damin, N., 1900: Pauci Dalmacije, Hrvatske, Slavonije i Istre. (Araneae Dalmatiae, Croatiae, Slavoniae et Istre partim editae, partim usque ad a. 1900 ineditae). Rad. Jugosl. Akad. Znan. Umj. 143, 10-53.

Deeleman-Reinhold, C. L. \& Deeleman, P. R., 1988: Revision des Dysderinae (Araneae, Dysderidae), les espèces mediterranéennes occidentales exceptées. Tijdschrift Voor Entomologie 131, 141-269.

Fortis, A., 1774: Viaggio in Dalmazia dell' abate Alberto Fortis (Vol. I-II). München -- Sarajevo: Verlag Otto Sagner - Veselin Masleša.

Gasperini, R., 1891: Prilog fauni dalmatinskih pauka (Araneae et Opiliones). Godišnje Izvješće C.K. Velike Realke u Splitu, 1-18.

Gasperini, R., 1892: Prilog k dalmatinskoj fauni (Isopoda, Myriapoda, Arachnida). God. Izvješće Vel. Realke za god. 1891./92., 1891./92., 1-22.

Hansen, H., 1995: Über die Arachniden-Fauna von urbanen Lebensraumen in Venedig-III. Die epigaischen Spinnen eines Stadtparkes (Arachnida: Araneae). Bolletino Di Museo Civico Di Storia Naturale Venezia 44, 183-219.

JurINAC, A. E., 1886: Faunistični pabirci po okolini krapinskoj. Glasn. Hrvats. Narav. Druž. 1, 145-153. Jocque, R., 2008: How to hydrate dried spiders. Newsletter of the British Arachnological Society 112, 5. JurinAC, A. E., 1887: Prilog hrvatskoj fauni ogulinsko-slunjske okolice i pećina. JAZU, 83(8), 86-128.

KATUŠić, L. \& DRAKŠić, M., 2011: How much do we know about Croatian spiders? - a historical overview of the literature. In P. Durbešić \& A. Previšić (Eds.), Entomologia Croatica \& Proceedings of the XXII Symposium Internationale Entomofaunisticum Europae Centralis (Vol. 15, pp. 237-254). Varaždin: Hrvatsko entomološko društvo.

Косн, L. C. C., 1867: Zur Arachniden- und Myriapoden-Fauna Süd-Europas. Verhandlungen Der Kaiserlich-Königlichen Zoologisch-Botanischen Gesellschaft in Wien 17, 857-900. Retrieved from http://books.google.hr/books?id=yG8oAAAAYAAJ

Kratochvíl, J. \& Miller, F., 1940: Neue Höhlenspinnen der Gattung Tegenaria aus Jugoslawien. Zool. Anz. 131, 188-201.

Langhoffer, A., 1906: Narcis Damin [nekrolog]. Glasn. Hrvats. narav. druž. 18, 78-80.

Le Peru, B., 2011: The Spiders of Europe, A Synthesis of Data: Vol. 1 Atypidae to Theridiidae. Lyon: Société Linnéenne. Retrieved from http://books.google.hr/books?id=ki9yuAAACAAJ

Levy, G., 1985: Fauna Palaestina. Arachnida II. Araneae: Thomisidae. The Israel Academy of Sciences and Humanities.

Mclean, B.S., Bell, K.C., Dunnum, J.L., Abrahamson, B, Colella, J.P., Deardorff, E.R., Weber, J., Jones, A.K., Salazar-Miralles, F. \& CooK, J.A., 2016: Natural history collections-based research: 
progress, promise and best practices. Journal of Mammalogy 97, 287-297. doi:10.1093/jmammal/ gyv178

Mammola, S., Cardoso, P., Ribera, C., Pavlek, M. \& Isaia, M., 2017: A synthesis on cave-dwelling spiders in Europe. J Zool Syst Evol Res.; 00: 1-16. https://doi.org/10.1111/jzs.12201

Metzner, H., 2013: Worldwide database of jumping spiders (Arachnida, Araneae, Salticidae). Retrieved from http://www.jumping-spiders.com/index.html

Simon, E. L., 1914: Les Arachnides de France. Synopsis général et Catalogue des espèces françaises de l'ordre des Araneae (Vol. 6). Paris: Librairie encyclopédique de Roret.

Sunarez, A. V. \& N. D. Tsutsui, 2004: The Value of Museum Collections for Research and Society. Bioscience. Vol. 54, No.1.

Tongrongi, P., 1966: Wolf Spiders of the Pardosa monticola Group (Araneae, Lycosidae). Bulletin of the Museum of Comparative Zoology 134(9), 335-359.

UтоснкіN, A. S., 1960: Spiders of the genus Synaema, the group globosum (F.) in the USSR. Zool. Zh. 39(7), 1018-1024.

UtochкIN, A. S., 1960: Spiders of the genus Synaema, the group plorator (O.P. Cambr.) in the USSR. Zool. Zh. 39(3), 375-380.

WINKER, K., 2004: Natural history museums in a postbiodiversity era. BioScience 54, 455-459.

WSC, 2018: World Spider Catalog. Version 20.0. Natural History Museum Bern, online at http://wsc. nmbe.ch, accessed on 14 December, 2018; doi: 10.24436/2 
Appendix 1. List of collecting localities of spiders from the CHNM Collection.

\begin{tabular}{|c|c|c|c|c|}
\hline Number & State & Locality & $x$ & $\mathbf{Y}$ \\
\hline 1 & Croatia & Vrgorac & 17,37218 & 43,20564 \\
\hline 2 & Croatia & Bađinec & 16,55952 & 45,8172 \\
\hline 3 & Croatia & Dalmacija & & \\
\hline 4 & Croatia & Novigrad, Dalmacija & 15,54885 & 44,18132 \\
\hline 5 & Croatia & Sveti Filip i Jakov, Dalmacija & 15,42846 & 43,96216 \\
\hline 6 & Croatia & Vrana, Dalmacija & 15,5673 & 43,9485 \\
\hline 7 & Croatia & špilja Pećina kod Vrane, Vrana, Dalmacija & 15,55492 & 43,9599 \\
\hline 8 & Croatia & Muć, Dalmatinska zagora & 16,47607 & 43,69165 \\
\hline 9 & Croatia & Dugi otok, luka Tajer & 15,20545 & 43,85303 \\
\hline 10 & Croatia & Đurđevački pijesci & 17,09927 & 46,02851 \\
\hline 11 & Croatia & Lokve, Gorski kotar & 14,75087 & 45,35831 \\
\hline 12 & Croatia & Mrzla Vodica, Lokve, Gorski kotar & 14,66558 & 45,36986 \\
\hline 13 & Croatia & Zapeć, špilja Zapeć, Gorski kotar & 15,08004 & 45,48199 \\
\hline 14 & Croatia & Vrata, Gorski kotar & 14,73011 & 45,31814 \\
\hline 15 & Croatia & Plešce, Gorski kotar & 14,68631 & 45,54669 \\
\hline 16 & Croatia & Bakar & 14,53425 & 45,30675 \\
\hline 17 & Croatia & Bakar, brdo Kalvarija & 14,53175 & 45,30564 \\
\hline 18 & Croatia & Bakar, iznad kupališta & 14,56709 & 45,29248 \\
\hline 19 & Croatia & Sveti Kuzam, Bakar & 14,52205 & 45,31459 \\
\hline 20 & Croatia & Bakar, obala & 14,54345 & 45,29795 \\
\hline 21 & Croatia & Bakar, područje Turčina & 14,53314 & 45,31043 \\
\hline 22 & Croatia & Bakar, predio Mandrać & 14,54283 & 45,29947 \\
\hline 23 & Croatia & Biograd na Moru, okolica & 15,44383 & 43,93721 \\
\hline 24 & Croatia & Cavtat & 18,2177 & 42,58116 \\
\hline 25 & Croatia & Crikvenica & 14,69124 & 45,17347 \\
\hline 26 & Croatia & Draga Crikvenička, Crikvenica & 14,71715 & 45,17252 \\
\hline 27 & Croatia & Selce, Crikvenica & 14,71976 & 45,15753 \\
\hline 28 & Croatia & Delnice & 14,80163 & 45,39879 \\
\hline 29 & Croatia & Dubrovnik & 18,09442 & 42,65063 \\
\hline 30 & Croatia & Đakovo & 18,40979 & 45,30994 \\
\hline 31 & Croatia & Đurđevac & 17,07144 & 46,03977 \\
\hline 32 & Croatia & Fužine & 14,71482 & 45,30494 \\
\hline 33 & Croatia & Garešnica & 16,94076 & 45,57417 \\
\hline 34 & Croatia & Hrvatska Kostajnica, područje Palanka & 16,53961 & 45,22798 \\
\hline 35 & Croatia & Hrvatska Kostajnica, rukavac Unčica & 16,54947 & 45,22031 \\
\hline 36 & Croatia & Karlobag & 15,07288 & 44,52842 \\
\hline 37 & Croatia & Klanjec & 15,7445 & 46,0506 \\
\hline 38 & Croatia & Kraljevica & 14,57176 & 45,27462 \\
\hline 39 & Croatia & Krapina & 15,8724 & 46,16043 \\
\hline
\end{tabular}




\begin{tabular}{|c|c|c|c|c|}
\hline Number & State & Locality & $x$ & $\mathbf{Y}$ \\
\hline 40 & Croatia & Križevci & 16,54628 & 46,02229 \\
\hline 41 & Croatia & grad Lovran & 14,27236 & 45,29688 \\
\hline 42 & Croatia & Malinska & 14,5288 & 45,12501 \\
\hline 43 & Croatia & Novi Vinodolski & 14,78976 & 45,12844 \\
\hline 44 & Croatia & Novi Vinodolski, izvor Ivanj & 14,78644 & 45,14535 \\
\hline 45 & Croatia & Ogulin & 15,22387 & 45,26582 \\
\hline 46 & Croatia & Ogulin, područje Bukovnik & 15,21697 & 45,2544 \\
\hline 47 & Croatia & $\operatorname{grad} \mathrm{Omiš}$ & 16,6929 & 43,44329 \\
\hline 48 & Croatia & Bregi, Opatija & 14,29882 & 45,3491 \\
\hline 49 & Croatia & Osijek & 18,69552 & 45,55487 \\
\hline 50 & Croatia & Otočac & 15,23752 & 44,86888 \\
\hline 51 & Croatia & Ozalj, Ozaljska špilja & 15,46563 & 45,61562 \\
\hline 52 & Croatia & Požega & 17,67447 & 45,33145 \\
\hline 53 & Croatia & Rijeka & 14,4422 & 45,32701 \\
\hline 54 & Croatia & Rijeka, Orehovica, područje Žakalj & 14,46491 & 45,33894 \\
\hline 55 & Croatia & Rijeka, Ratulje & 14,44562 & 45,38505 \\
\hline 56 & Croatia & Rijeka, Sušak & 14,47194 & 45,32402 \\
\hline 57 & Croatia & Rijeka, Trsat & 14,46656 & 45,33334 \\
\hline 58 & Croatia & Rovinj & 13,63875 & 45,08114 \\
\hline 59 & Croatia & Samobor & 15,71113 & 45,80099 \\
\hline 60 & Croatia & Senj & 14,90359 & 44,98931 \\
\hline 61 & Croatia & Senj, okolica & 14,90722 & 44,99094 \\
\hline 62 & Croatia & Skradin & 15,92326 & 43,81755 \\
\hline 63 & Croatia & Skradin, uvala Rokovača, Stari Banj & 15,92153 & 43,81814 \\
\hline 64 & Croatia & Solin & 16,49191 & 43,54221 \\
\hline 65 & Croatia & Split & 16,44018 & 43,50808 \\
\hline 66 & Croatia & Split, Vranjic & 16,46252 & 43,53171 \\
\hline 67 & Croatia & Šibenik & 15,89522 & 43,73487 \\
\hline 68 & Croatia & Vrpolje, Šibenik & 16,01166 & 43,67531 \\
\hline 69 & Croatia & Topusko & 15,97536 & 45,29618 \\
\hline 70 & Croatia & Trogir & 16,24969 & 43,51701 \\
\hline 71 & Croatia & Valpovo & 18,41556 & 45,659 \\
\hline 72 & Croatia & Vinkovci & 18,8057 & 45,28781 \\
\hline 73 & Croatia & Zadar & 15,23135 & 44,11888 \\
\hline 74 & Croatia & Zadar, Arbanasi & 15,2388 & 44,1045 \\
\hline 75 & Croatia & Zagreb & 15,97529 & 45,81171 \\
\hline 76 & Croatia & Zagreb, Dubravkin put & 15,9733 & 45,81864 \\
\hline 77 & Croatia & Brezovica, Zagreb & 15,91066 & 45,72921 \\
\hline 78 & Croatia & Zagreb, Cmrok & 15,97243 & 45,83704 \\
\hline
\end{tabular}




\begin{tabular}{|c|c|c|c|c|}
\hline Number & State & Locality & $x$ & $\mathbf{Y}$ \\
\hline 79 & Croatia & Zagreb, Dubrava & 16,0596 & 45,82936 \\
\hline 80 & Croatia & Zagreb, Gračani & 15,97217 & 45,8586 \\
\hline 81 & Croatia & Zagreb, Ksaver & 15,97699 & 45,83135 \\
\hline 82 & Croatia & Zagreb, Podsused & 15,84357 & 45,82184 \\
\hline 83 & Croatia & Zagreb, Prečko & 15,90027 & 45,79036 \\
\hline 84 & Croatia & Zagreb, Remete & 15,99379 & 45,84537 \\
\hline 85 & Croatia & Zagreb, Šestine & 15,95003 & 45,85274 \\
\hline 86 & Croatia & Zagreb, Tuškanac & 15,97109 & 45,82558 \\
\hline 87 & Croatia & Zagreb, park Maksimir & 16,01566 & 45,8196 \\
\hline 88 & Croatia & Hrvatsko primorje & & \\
\hline 89 & Croatia & Korenići, Istra & 13,80116 & 45,12997 \\
\hline 90 & Croatia & između Bakra i otoka Košljuna & 14,54639 & 45,15716 \\
\hline 91 & Croatia & između Bakra i uvale Martinšćice & 14,48288 & 45,31335 \\
\hline 92 & Croatia & između Bakra i Crikvenice & 14,66503 & 45,20063 \\
\hline 93 & Croatia & između Bakra i Kraljevice & 14,56509 & 45,28115 \\
\hline 94 & Croatia & između Bakra i Omišlja & 14,57302 & 45,24921 \\
\hline 95 & Croatia & između Bakra i Senja & 14,74204 & 45,14798 \\
\hline 96 & Croatia & između Rijeke i Crikvenice & 14,60511 & 45,26674 \\
\hline 97 & Croatia & između Zlobina i Liča & 14,67595 & 45,27628 \\
\hline 98 & Croatia & Bakarac & 14,58274 & 45,28013 \\
\hline 99 & Croatia & Donja Zdenčina & 15,76222 & 45,6678 \\
\hline 100 & Croatia & Jadovno & 15,22062 & 44,54485 \\
\hline 101 & Croatia & Pisarovina & 15,85918 & 45,58796 \\
\hline 102 & Croatia & Zečev Varoš, nepoznata špilja & 15,54332 & 45,13443 \\
\hline 103 & Croatia & Moslavina & 17,98182 & 45,78537 \\
\hline 104 & Croatia & Božjakovina & 16,286 & 45,81692 \\
\hline 105 & Croatia & Grižane-Belgrad & 14,71843 & 45,20257 \\
\hline 106 & Croatia & Jablanac & 14,89802 & 44,70652 \\
\hline 107 & Croatia & Kaštel Žegarski, špilja Golubnjača & 15,85167 & 44,13176 \\
\hline 108 & Croatia & Križišće & 14,61103 & 45,26075 \\
\hline 109 & Croatia & Lukovo Otočko & 14,89411 & 44,85451 \\
\hline 110 & Croatia & Povile & 14,81981 & 45,1164 \\
\hline 111 & Croatia & Starigrad Paklenica & 15,43874 & 44,29583 \\
\hline 112 & Croatia & Sveti Juraj & 14,92019 & 44,92834 \\
\hline 113 & Croatia & Sveti Juraj, područje Borova Draga & 14,93622 & 44,9312 \\
\hline 114 & Croatia & NP Risnjak, livada Lazac, 1069 m n.m. & 14,60159 & 45,45014 \\
\hline 115 & Croatia & Babina Greda & 18,53701 & 45,1167 \\
\hline
\end{tabular}




\begin{tabular}{|c|c|c|c|c|}
\hline Number & State & Locality & $x$ & $\mathbf{Y}$ \\
\hline 116 & Croatia & Jelenje & 14,45059 & 45,38807 \\
\hline 117 & Croatia & Kostrena & 14,51767 & 45,30036 \\
\hline 118 & Croatia & Šoići, Kostrena & 14,54186 & 45,28999 \\
\hline 119 & Croatia & Sveta Barbara, Kostrena & 14,53476 & 45,2907 \\
\hline 120 & Croatia & Sveta Lucija, Kostrena & 14,49754 & 45,30714 \\
\hline 121 & Croatia & uvala Martinšćica, Kostrena & 14,48129 & 45,31205 \\
\hline 122 & Croatia & Kraljevica, naselje Dol & 14,61981 & 45,2554 \\
\hline 123 & Croatia & Brešca, Matulji & 14,29458 & 45,4096 \\
\hline 124 & Croatia & Trgetari, Raša & 14,06743 & 45,01431 \\
\hline 125 & Croatia & Viljevo & 18,06325 & 45,75136 \\
\hline 126 & Croatia & otočić Čavatul & 15,41193 & 43,93831 \\
\hline 127 & Croatia & otok Babac & 15,40422 & 43,95562 \\
\hline 128 & Croatia & otok Biševo & 16,01129 & 42,97771 \\
\hline 129 & Croatia & otok Hvar & 16,64214 & 43,15287 \\
\hline 130 & Croatia & Stari grad, otok Hvar & 16,59926 & 43,18395 \\
\hline 131 & Croatia & otok Hvar, selo Brusje & 16,48848 & 43,19173 \\
\hline 132 & Croatia & otok Košljun & 14,61891 & 45,02591 \\
\hline 133 & Croatia & otok Krk & 14,59257 & 45,08088 \\
\hline 134 & Croatia & Krk, naselje Lunta, otok Krk & 14,56662 & 45,02093 \\
\hline 135 & Croatia & Omišalj, otok Krk & 14,55692 & 45,21098 \\
\hline 136 & Croatia & Omišalj, groblje Sv. Duh, otok Krk & 45,199785 & 14,56051 \\
\hline 137 & Croatia & Baška, otok Krk & 14,7522 & 44,97102 \\
\hline 138 & Croatia & Glavotok, otok Krk & 14,4438 & 45,08922 \\
\hline 139 & Croatia & Polje, otok Krk & 14,64371 & 45,13552 \\
\hline 140 & Croatia & Punat, otok Krk & 14,63161 & 45,02069 \\
\hline 141 & Croatia & Rudine, otok Krk & 14,61202 & 45,18456 \\
\hline 142 & Croatia & Sveti Ivan Dobrinjski, otok Krk & 14,58468 & 45,12548 \\
\hline 143 & Croatia & Voz, otok Krk & 14,57958 & 45,23192 \\
\hline 144 & Croatia & otok Krk, okolica jezera Ponikve & 14,56947 & 45,07402 \\
\hline 145 & Croatia & otok Krk, područje Brgud & 14,57761 & 45,08233 \\
\hline 146 & Croatia & otok Krk, selo Rudine, špilja Biserujka & 14,60977 & 45,18743 \\
\hline 147 & Croatia & otok Lokrum & 18,11757 & 42,63096 \\
\hline 148 & Croatia & Polače, otok Mljet & 17,37772 & 42,78524 \\
\hline 149 & Croatia & otok Pag & 15,09154 & 44,43229 \\
\hline 150 & Croatia & Novalja, otok Pag & 14,88544 & 44,55616 \\
\hline 151 & Croatia & Novalja, područje Špital, otok Pag & 14,88001 & 44,54516 \\
\hline 152 & Croatia & Velo Blato, otok Pag & 15,1565 & 44,35602 \\
\hline
\end{tabular}




\begin{tabular}{|c|c|c|c|c|}
\hline Number & State & Locality & $x$ & $\mathrm{Y}$ \\
\hline 153 & Croatia & Kolansko blato, otok Pag & 14,91728 & 44,5206 \\
\hline 154 & Croatia & Caska, otok Pag & 14,91964 & 44,55065 \\
\hline 155 & Croatia & Kolan, otok Pag & 14,95917 & 44,49653 \\
\hline 156 & Croatia & Povljana, otok Pag & 15,11279 & 44,34613 \\
\hline 157 & Croatia & Stara Novalja, otok Pag & 14,87664 & 44,59648 \\
\hline 158 & Croatia & otok Pag, područje Barbat & 15,00374 & 44,51398 \\
\hline 159 & Croatia & otok Palagruža & 16,25946 & 42,39211 \\
\hline 160 & Croatia & otok Pašman & 15,36125 & 43,94463 \\
\hline 161 & Croatia & otok Rab & 14,75193 & 44,78166 \\
\hline 162 & Croatia & otok Susak & 14,30379 & 44,50764 \\
\hline 163 & Croatia & otok Sveti Marko & 14,56229 & 45,25252 \\
\hline 164 & Croatia & otok Unije & 14,25067 & 44,63175 \\
\hline 165 & Croatia & otok Unije, Unijsko polje & 14,24716 & 44,63374 \\
\hline 166 & Croatia & otok Vis & 16,15407 & 43,04584 \\
\hline 167 & Croatia & Komiža, otok Vis & 16,0918 & 43,04445 \\
\hline 168 & Croatia & Park šuma Golubinjak, Golubinja špilja & 14,76617 & 45,35576 \\
\hline 169 & Croatia & Ivanšćica & 16,13232 & 46,18138 \\
\hline 170 & Croatia & Kalnik & 16,45459 & 46,13128 \\
\hline 171 & Croatia & Klek & 15,1454 & 45,26091 \\
\hline 172 & Croatia & Medvednica & 15,95268 & 45,9093 \\
\hline 173 & Croatia & Medvednica, izvor Kraljičin zdenac & 15,94205 & 45,88177 \\
\hline 174 & Croatia & Medvednica, vrh Lipa & 16,06926 & 45,93028 \\
\hline 175 & Croatia & Medvednica, vrh Sljeme & 15,97564 & 45,92636 \\
\hline 176 & Croatia & Papuk, Jankovac & 17,6868 & 45,52185 \\
\hline 177 & Croatia & Risnjak & 14,61465 & 45,44951 \\
\hline 178 & Croatia & Risnjak, Crni Lug & 14,70479 & 45,4184 \\
\hline 179 & Croatia & Risnjak, od 1000 do 1500 m n.m. & 14,61988 & 45,4319 \\
\hline 180 & Croatia & Velebit, između Grabarja i Alana & 14,95307 & 44,70547 \\
\hline 181 & Croatia & Vratnik, Velebit & 15,00684 & 44,97828 \\
\hline 182 & Croatia & Velebit, Mrkvište & 15,04838 & 44,70893 \\
\hline 183 & Croatia & Velika Kapela, Bitoraj & 14,76563 & 45,28941 \\
\hline 184 & Croatia & Sošice, Žumberak & 15,38083 & 45,75071 \\
\hline 185 & Croatia & Stari grad Žumberački, Kekići, Žumberak & 15,41116 & 45,76107 \\
\hline 186 & Croatia & Žumberak, Sveta Gera & 15,32082 & 45,75997 \\
\hline 187 & Croatia & Plitvička jezera & 15,62192 & 44,88113 \\
\hline 188 & Croatia & Plitvička jezera, Jasikovac & 15,72365 & 44,72519 \\
\hline 189 & Croatia & Crna Mlaka & 15,73248 & 45,60889 \\
\hline
\end{tabular}




\begin{tabular}{|c|c|c|c|c|}
\hline Number & State & Locality & $X$ & Y \\
\hline 190 & Croatia & Pazarište & 15,19945 & 44,65138 \\
\hline 191 & Croatia & Krka, Skradinski buk & 15,96414 & 43,80516 \\
\hline 192 & Croatia & Ombla & 18,13641 & 42,67473 \\
\hline 193 & Croatia & Rječina & 14,44002 & 45,37394 \\
\hline 194 & Croatia & Rječina, izvor & 14,42444 & 45,42475 \\
\hline 195 & Croatia & Krasica & 14,55735 & 45,31128 \\
\hline 196 & Croatia & Mošćenice & 14,24754 & 45,22628 \\
\hline 197 & Croatia & Sikirevci & 18,46477 & 45,10838 \\
\hline 198 & Croatia & Turopolje & 16,1394 & 45,64825 \\
\hline 199 & Croatia & Vinodol & 14,74525 & 45,23095 \\
\hline 200 & Croatia & Bribir, Vinodol & 14,75969 & 45,16201 \\
\hline 201 & Croatia & Vransko polje, područje uz kanal Vrbica & 15,50709 & 43,94568 \\
\hline 202 & Croatia & Zrinska gora, Dodoši & 16,28396 & 45,30499 \\
\hline 203 & Austria & Hundsberg & 16,28334 & 48,33331 \\
\hline 204 & $\begin{array}{l}\text { Bosnia and } \\
\text { Herzegovina }\end{array}$ & Bosnia and Herzegovina & & \\
\hline 205 & Montenegro & Budva & 18,8403 & 42,29101 \\
\hline 206 & Montenegro & Kotor & 18,77124 & 42,42466 \\
\hline 207 & Montenegro & Kotor, brdo Vrmac & 18,72003 & 42,45002 \\
\hline 208 & Montenegro & Muo & 18,75118 & 42,4298 \\
\hline 209 & India & grad Mumbai & 72,87754 & 19,076 \\
\hline 210 & South America & South America & & \\
\hline 211 & Hungary & Budimpešta & 19,04018 & 47,4978 \\
\hline 212 & Hungary & Kecskemet & 19,6897 & 46,89634 \\
\hline 213 & Hungary & Sátoraljaújhely & 21,65511 & 48,39601 \\
\hline 214 & Hungary & Martinka & 21,76104 & 47,56629 \\
\hline 215 & Poland & Krakow & 19,94505 & 50,06465 \\
\hline 216 & Romania & Cluj-Napoca & 23,62369 & 46,77059 \\
\hline 217 & Slovakia & Visoke Tatre & 20,13166 & 49,16602 \\
\hline 218 & Slovenia & Kamnik, dvorac Mali grad & 14,61137 & 46,22455 \\
\hline 219 & Slovenia & Kralji & 15,03583 & 45,53739 \\
\hline 220 & Serbia & Beočin & 19,72212 & 45,19674 \\
\hline 221 & Serbia & Petrovaradin & 19,88617 & 45,23608 \\
\hline 222 & Serbia & Sombor & 19,11516 & 45,77324 \\
\hline 223 & Serbia & Vrbas & 19,64496 & 45,57006 \\
\hline 224 & Serbia & Zemun & 20,35574 & 44,85291 \\
\hline 225 & Serbia & Bešenovo & 19,7001 & 45,08317 \\
\hline 226 & Serbia & Boljevci & 20,22311 & 44,72174 \\
\hline
\end{tabular}




\begin{tabular}{|l|l|l|l|l|}
\hline Number & State & Locality & X & Y \\
\hline 227 & Serbia & Jarak & 19,75747 & 44,91649 \\
\hline 228 & Serbia & Srijemska Mitrovica & 19,62096 & 44,97926 \\
\hline 229 & Serbia & Stara Pazova & 20,15695 & 44,98554 \\
\hline 230 & Serbia & Stari Vrbas & 19,66705 & 45,56105 \\
\hline 231 & Serbia & Ššatovac & 19,55276 & 45,12061 \\
\hline 232 & Serbia & Vrdnik & 19,78972 & 45,12869 \\
\hline 233 & Serbia & Obedska bara & 19,99435 & 44,70663 \\
\hline 234 & Serbia & Fruška Gora & 19,70938 & 45,15691 \\
\hline 235 & Serbia & Čerević & 19,66671 & 45,21668 \\
\hline 236 & Serbia & Kupinovo & 20,04835 & 44,70532 \\
\hline 237 & Serbia & Nikoličevo & 22,23694 & 43,9605 \\
\hline 238 & Serbia & Subotište & 19,96149 & 44,84753 \\
\hline 239 & Ukraine & Galicija & & \\
\hline
\end{tabular}




\section{Appendix 2.}

Spider species in the CNHM Collection of Spiders considered as new species in Croatian fauna, including species whose identity could not be confirmed, in alphabetical order.

Abbreviations:

* - new species to the Croatian fauna.

*? - dubious records.

** - species represented by damaged specimen, thus their re-identification was

not possible. Such species names are listed in their current taxonomic interpretation based on the original identification; if a re-examination did not confirm the species name given originally, such samples are listed under generic names only.

*** - Species represented only with juvenile specimens, thus redetermination was possible only to the genus level. The stated species name is current taxonomic interpretation of the original species name. \# - quotations the Museum's Spider Catalogue without specimen.

Family A GELENIDAE

Tegenaria ferruginea (Panzer, 1804)*

Textrix chyzeri de Blauwe, $1980^{*}$

Family AMAUROBIIDAE

Amaurobius jugorum L. Koch, $1868^{*}$

Family ARANEIDAE

Araniella inconspicua (Simon, 1874) ${ }^{* * * *}$

Zygiella atrica (C. L. Koch, 1845)*

Family CLUBIONIDAE

(Clubiona decora Blackwall, 1859 ****)

Clubiona frutetorum L. Koch, $1867^{* *}$

Dysderocrates storkani (Kratochvíl, 1935)*

\section{Family EUTICHURIDAE}

Cheiracanthium elegans Thorell, $1875^{*, * * *}$

Cheiracanthium punctorium (Villers, 1789)*

Family GNAPHOSIDAE

Berinda sp. nov.*? (Katušić in prep.)

Micaria pallipes (Lucas, 1846)*

Family LINYPHIIDAE

Micrargus herbigradus (Blackwall, 1854)*

Neriene peltata (Wider, 1834 )*

Silometopus reussi (Thorell, 1871)*

Stemonyphantes lineatus (Linnaeus, 1758) *****

Family LIOCRANIDAE

Walckenaeria antica (Wider, 1834) **

Family LYCOSIDAE

Alopecosa farinosa (Herman, 1879)*

Alopecosa sulzeri (Pavesi, 1873)*
Alopecosa taeniata (C. L. Koch, 1835)*

Pardosa alacris (C. L. Koch, 1833)*

Trochosa terricola Thorell, $1856^{*}$

Family PHILODROMIDAE

Philodromus marmoratus Kulczyński, 1891 *,***

Thanatus coloradensis Keyserling, 1880*

Family SALTICIDAE

Dendryphantes rudis (Sundevall, 1833) , $* * * *^{*}$

Mendoza canestrinii (Ninni, 1868) *****

Neon reticulatus (Blackwall, 1853) **

Sitticus pubescens (Fabricius, 1775)*

Family TETRAGNATHIDAE

Tetragnatha montana Simon, $1874^{*}$

Tetragnatha nigrita Lendl, $1886^{*}$

Family THERIDIID AE

Parasteatoda simulans (Thorell, 1875)*

Phylloneta sisyphia (Clerck, 1757)*

Robertus arundineti (O. P.-Cambridge, 1871)*

Theridion mystaceum L. Koch, $1870^{*}$

Family THOMISID AE

Synema cf. ornatum (Thorell, 1875) sensu (Utochkin A. S., 1960)*?

Xysticus bifasciatus C. L. Koch, $1837^{*}$

Xysticus erraticus (Blackwall, 1834)*

Xysticus gallicus Simon, 1875*

Xysticus tenebrosus Silhavy, 1944*

Family ZOD ARIID AE

Zodarion germanicum (C. L. Koch, 1837) ${ }^{* * * * *}$ 
